\title{
SUBLIMATION ENTHALPIES OF ORGANIC COMPOUNDS: A VERY LARGE DATABASE WITH A MATCH TO CRYSTAL STRUCTURE DETERMINATIONS AND A COMPARISON WITH LATTICE ENERGIES
}

James S. Chickos ${ }^{1}$ and Angelo Gavezzotti ${ }^{2}$

${ }^{1}$ Department of Chemistry, University of Missouri-St. Louis, Saint Louis, Missouri 63121 e-mail: jsc@umsl.edu

${ }^{2}$ Dipartimento di Chimica, Università di Milano, via Venezian 21, 20133 Milano, Italy e-mail: angelo.gavezzotti@unimi.it

\section{Correction-RT: Vibrational energy corrections of sublimation enthalpies for comparison with lattice energies: invalid approximations}

The lattice energy ( $\mathrm{LE}, \mathrm{kJ} \mathrm{mol}^{-1}$ ) for a one-component crystal is the difference in calculated internal energy between one mole of constituent molecules at infinite separation and one mole of the same constituents in the crystal. If infinite separation in the gas phase with zero interaction energy is taken as a reference, LE is by definition stabilizing and coincides with the thermodynamic internal intermolecular potential energy, $\mathrm{U}($ interm, cry $)=\mathrm{LE}$. Please note carefully that stabilizing/destabilizing refer to energies being $<0$ or $>0$, respectively, while attractive/repulsive refer to forces acting to decrease or increase separation, respectively. ${ }^{\text {ag }}$

Thermodynamic internal energy also has translational and rotational kinetic terms and vibrational terms. For the gas and liquid phases the complete expressions are:

$$
\begin{aligned}
& \mathrm{U}(\text { tot, gas })=\mathrm{U}(\text { interm, gas })+\mathrm{E}(\text { kin, trasl })+\mathrm{E}(\text { kin, rot })+\mathrm{E}(\text { vib, intram }) \\
& \mathrm{U}(\text { tot, liq })=\mathrm{U}(\text { interm, liq })+\mathrm{E}(\text { kin, trasl })+\mathrm{E}(\text { kin, rot })+\mathrm{E}(\text { vib, intram })
\end{aligned}
$$

where $\mathrm{E}$ (vib,intram) denotes the intramolecular vibrational energy. In the crystal full translational and full rotational energies are suppressed because there is no net motion of the center of mass and no net overall molecular rotation; these terms are replaced by potential end kinetic terms for translational and rotational librations (t-lib and r-lib):

$$
\mathrm{U}(\text { tot, cry })=\mathrm{U}(\text { interm, cry })+\mathrm{E}(\mathrm{t}-\mathrm{lib}, \mathrm{kin})+\mathrm{E}(\mathrm{t}-\mathrm{lib}, \text { pot })+\mathrm{E}(\mathrm{r}-\mathrm{lib}, \mathrm{kin})+\mathrm{E}(\mathrm{r}-\mathrm{lib} \text {.pot })+\mathrm{E}(\text { vib,intram })
$$

Let us first assume that $\mathrm{E}$ (vib,intram) is the same in all aggregation states (a far-fetched assumption except for very tightly bound molecules, benzene and little else) and can therefore be 
neglected when taking energy differences. U(interm,gas) is assumed as zero for the very dilute (low-pressure) vapors of a solid at room temperature (semi-ideal gas assumption). In the harmonic equipartition regime each energy term is worth $3 / 2 \mathrm{RT}$, therefore one gets:

$\mathrm{U}($ tot,gas $)=3 \mathrm{RT}$

$\mathrm{U}($ tot, $\mathrm{liq})=\mathrm{U}($ interm, $\mathrm{liq})+3 \mathrm{RT}$

$\mathrm{U}($ tot,cry $)=\mathrm{U}($ interm, cry $)+6 \mathrm{RT}$

Recalling now that $\mathrm{H}=\mathrm{U}+\mathrm{PV}$ and writing the phase transition functions one gets the following approximate expressions at constant pressure:

vaporization enthalpy:

$\Delta \mathrm{H}($ gas-liq $)=\Delta \mathrm{U}($ gas-liq $)+\mathrm{P} \Delta \mathrm{V}=\Delta \mathrm{U}($ gas-liq $)+\mathrm{PV}($ gas $)=\Delta \mathrm{U}($ gas-liq $)+\mathrm{RT}=-\mathrm{U}($ liq $)+\mathrm{RT}$ where the volume of the liquid is negligible against the volume of the (ideal) gas;

melting enthalpy:

$\Delta \mathrm{H}($ liq-cry $)=\Delta \mathrm{U}($ liq-cry $)+\mathrm{P} \Delta \mathrm{V}=\Delta \mathrm{U}($ liq-cry $)=\mathrm{U}($ liq $)-\mathrm{U}($ cry $)-3 \mathrm{RT}$

where the volume change on melting is negligible;

sublimation enthalpy

$\Delta \mathrm{H}($ gas-cry $)=\Delta \mathrm{U}($ gas-cry $)+\mathrm{P} \Delta \mathrm{V}=\Delta \mathrm{U}($ gas-cry $)+\mathrm{RT}=3 \mathrm{RT}-\mathrm{U}($ cry $)-6 \mathrm{RT}+\mathrm{RT}=-\mathrm{U}($ cry $)-2 \mathrm{RT}$ or $\mathrm{SE}=-\mathrm{LE}-2 \mathrm{RT}$

Another way of seeing the matter is to assign a constant heat capacity of $3 R$ to the gas and of $6 R$ to the solid, and to perform the standard thermodynamic integration:

$\Delta \mathrm{Us}(\mathrm{T})=\Delta \mathrm{Us}(0)+\int_{(0-\mathrm{T})} \Delta \mathrm{CvdT}=\Delta \mathrm{Us}(0)-3 \mathrm{R} \int_{(0-\mathrm{T})} \mathrm{dT}=\Delta \mathrm{Us}(0)-3 \mathrm{RT}$

$\Delta \mathrm{Hs}=\Delta \mathrm{Us}+\Delta(\mathrm{PV})=\Delta \mathrm{Us}+\mathrm{PV}=\Delta \mathrm{Us}+\mathrm{RT}=\Delta \mathrm{Us}(0)-2 \mathrm{RT}$

whereby the calculated LE is seen as the sublimation internal energy at $0 \mathrm{~K}$. Here the assumption of constant $\mathrm{Cv}$ goes against one of the basic laws of thermodynamics, namely the convergence to zero of solid heat capacities at low temperature. 
Table S1. 1655 reported sublimation enthalpies. First line: compound name; second line: brute formula, SE: assessed sublimation enthalpy, SEmin, max: range of experimental variation when not zero; $T$, temperature of the measurement, when zero, assumed at room T; Nmeas, number of accepted experimental measurements $(0=1)$. Attached to each entry is the refcode for the 737 corresponding crystal structure determinations in the Cambridge Structural Database. Each refcode may correspond to more than one crystal structure determination for the same compound.

\begin{tabular}{|c|c|c|c|c|c|c|c|}
\hline & & SE & SEMTn & SEmax & < $\mathrm{T}$ Nme & & CSD retcode \\
\hline 1 & cyanogen bromide & & & & & & \\
\hline $\mathrm{C} \perp \underset{2}{\mathrm{~B} r}$ & $\begin{array}{l}1 \\
\text { carbon } \\
\text { I }\end{array}$ & 46.0 & 45.0 & $4 / .0$ & 300.0 & 3 & CTBROM \\
\hline $\mathrm{C} 1 \underset{3}{\mathrm{Br}}$ & 4 cyanogen chloride & 54.4 & 49.4 & 54.4 & 300.0 & 2 & \\
\hline $\begin{array}{l}1 \mathrm{C} 1 \\
4\end{array}$ & $\begin{array}{l}1 \\
\text { diFluorotrichloromethy] }\end{array}$ & $\begin{array}{l}36.0 \\
\text { phosph }\end{array}$ & 0.0 & 0.0 & 230.0 & 1 & \\
\hline C 1 C 1 & $\begin{array}{lll}3 & 2 & P \\
\end{array}$ & 36.8 & 0.0 & 0.0 & 274.0 & 1 & \\
\hline C1 & 4 carbon tetrachioride & 41.0 & 38.0 & 43.0 & 220.0 & 3 & CARBTC \\
\hline C $1{ }_{7}^{0} \mathrm{~F}$ & $\begin{array}{l}\text { carbonyl Fluoride } \\
1 \text { N } 1 \\
\text { carbon }\end{array}$ & 29.0 & 0.0 & 0.0 & 170.0 & 2 & \\
\hline C 18 & 201 & 23.0 & 0.0 & 0.0 & 145.0 & 1 & \\
\hline C $1{ }_{9}^{F}$ & $\begin{array}{l}\text { carbon tetratiuoride } \\
4 \\
\text { pentaFluoromethy } 7 \text { amine }\end{array}$ & 16.0 & 14.0 & 17.0 & 80.0 & 5 & IFME I H \\
\hline C $1_{10} \mathrm{~F}$ & $\begin{array}{l}5 \mathrm{~N} 1 \\
\text { cyanogen iodide }\end{array}$ & 19.0 & 0.0 & 0.0 & 135.0 & 1 & \\
\hline$C 1_{11}^{+} \mathrm{I}$ & $\begin{array}{l}1 \mathrm{~N} 1 \\
\text { tetranitromethane }\end{array}$ & 59.0 & 58.0 & 60.0 & 350.0 & 4 & \\
\hline C $1_{12}^{1+} \mathrm{N}$ & $\begin{array}{l}408 \text { monide } \\
\text { carbon monoxid }\end{array}$ & 47.0 & 0.0 & 0.0 & 270.0 & 1 & \\
\hline $\mathrm{C} 1_{13}^{12} \mathrm{O}$ & 1 carbon dioxide & 8.1 & 8.0 & 8.2 & 61.5 & 2 & SACBAA \\
\hline$C 1_{14}^{10} 0$ & trifluoromethane & 26.1 & 25.2 & 27.2 & 190.0 & 5 & SACBAA \\
\hline $\mathrm{C}_{15} \mathrm{H}$ & $\begin{array}{l}1 \text { F } 3 \\
\text { iodoform }\end{array}$ & 26.0 & 0.0 & 0.0 & 100.0 & 1 & IODOFO \\
\hline C $1_{16} \mathrm{H}$ & $\begin{array}{l}1 \text { I } 3 \\
\text { hydrogen cyanide }\end{array}$ & 70.0 & 0.0 & 0.0 & 320.0 & 1 & \\
\hline C $1_{17} \mathrm{H}$ & $\begin{array}{l}1 \mathrm{~N} 1 \\
\text { trinitromethane }\end{array}$ & 36.0 & 35.6 & 37.6 & 240.0 & 2 & HEVRUV \\
\hline & $\begin{array}{l}1 \mathrm{~N} 306 \\
\text { cyanamide }\end{array}$ & 48.0 & 47.0 & 55.0 & 300.0 & 2 & CYANAM \\
\hline $\mathrm{C}_{19}{ }^{\mathrm{H}}$ & $\begin{array}{l}2 \mathrm{~N} 2 \\
\text { tetrazole }\end{array}$ & 75.0 & 0.0 & 0.0 & 300.0 & 2 & TETZOL \\
\hline & $\begin{array}{l}2 \mathrm{~N} 4 \\
\text { formic acid }\end{array}$ & 88.0 & 88.0 & 98.0 & 350.0 & 3 & FORMAC \\
\hline C $1_{21} \mathrm{H}$ & $\begin{array}{l}202 \\
\text { methy } 1 \text { chloride }\end{array}$ & 61.0 & 60.0 & 62.0 & 260.0 & 4 & CLMETH \\
\hline & $\begin{array}{l}3 \text { C1 } 1 \\
\text { methy } 1 \text { iodide }\end{array}$ & 32.0 & 0.0 & 0.0 & 150.0 & 1 & \\
\hline $\mathrm{C}_{23} \mathrm{H}$ & $\begin{array}{l}3 \quad \text { I } 1 \\
\text { formamide }\end{array}$ & 40.0 & 0.0 & 0.0 & 190.0 & 1 & EOPMAM \\
\hline C ${ }_{24} \mathrm{H}$ & $\begin{array}{l}3 \mathrm{~N} 1 \mathrm{O} \quad 1 \\
5 \text {-aminotetrazole }\end{array}$ & 72.0 & 0.0 & 0.0 & 300.0 & 3 & 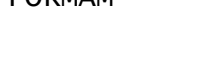 \\
\hline $\mathrm{C}_{25} \mathrm{H}$ & $\begin{array}{l}35 \\
\text { methane }\end{array}$ & 113.0 & 0.0 & 0.0 & 400.0 & 1 & \\
\hline $\mathrm{C}_{26} \mathrm{H}$ & $\begin{array}{l}4 \\
\text { urea }\end{array}$ & 10.0 & 9.2 & 10.0 & 80.0 & 5 & UREAXX \\
\hline C ${ }_{27} \mathrm{H}$ & 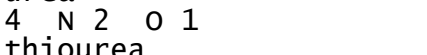 & 97.0 & 95.0 & 99.0 & 330.0 & 6 & THTOUR \\
\hline C $1_{28} \mathrm{H}$ & 4 N 2 S 1 & 108.0 & 104.0 & 112.0 & 330.0 & 7 & IHIUUR \\
\hline $\mathrm{C}_{29} \mathrm{H}$ & $\begin{array}{l}4 \mathrm{~N} 402 \\
N \text {-methy } 7 \text { hydroxylamine }\end{array}$ & 143.0 & 0.0 & 0.0 & 300.0 & 1 & NIRGUA \\
\hline $\mathrm{C}_{30} \mathrm{H}$ & $\begin{array}{l}5 \mathrm{~N} 1 \text { o } 1 \\
1 \text {-methy } 1-1-\text {-nitrosohydraz }\end{array}$ & $\begin{array}{l}57.0 \\
\text { zine }\end{array}$ & 0.0 & 0.0 & 290.0 & 1 & \\
\hline C $1_{31} \mathrm{H}$ & $\begin{array}{l}5 \text { N } 3 \text { O } 1 \\
\text { thiosemicarbazide }\end{array}$ & 80.0 & 0.0 & 0.0 & 300.0 & 1 & TSCRBZ \\
\hline $\mathrm{C}_{32} \mathrm{H}$ & $\begin{array}{l}5 \text { N } 3 \text { S } 1 \\
\text { thiocarbohydrazide }\end{array}$ & 126.0 & 0.0 & 0.0 & 300.0 & 1 & \\
\hline $\mathrm{C}_{33} \mathrm{H}$ & $\begin{array}{l}6 \mathrm{~N} 4 \mathrm{~S} 1 \\
\text { bromopentachloroethane }\end{array}$ & 152.0 & 0.0 & 0.0 & 300.0 & 1 & \\
\hline
\end{tabular}




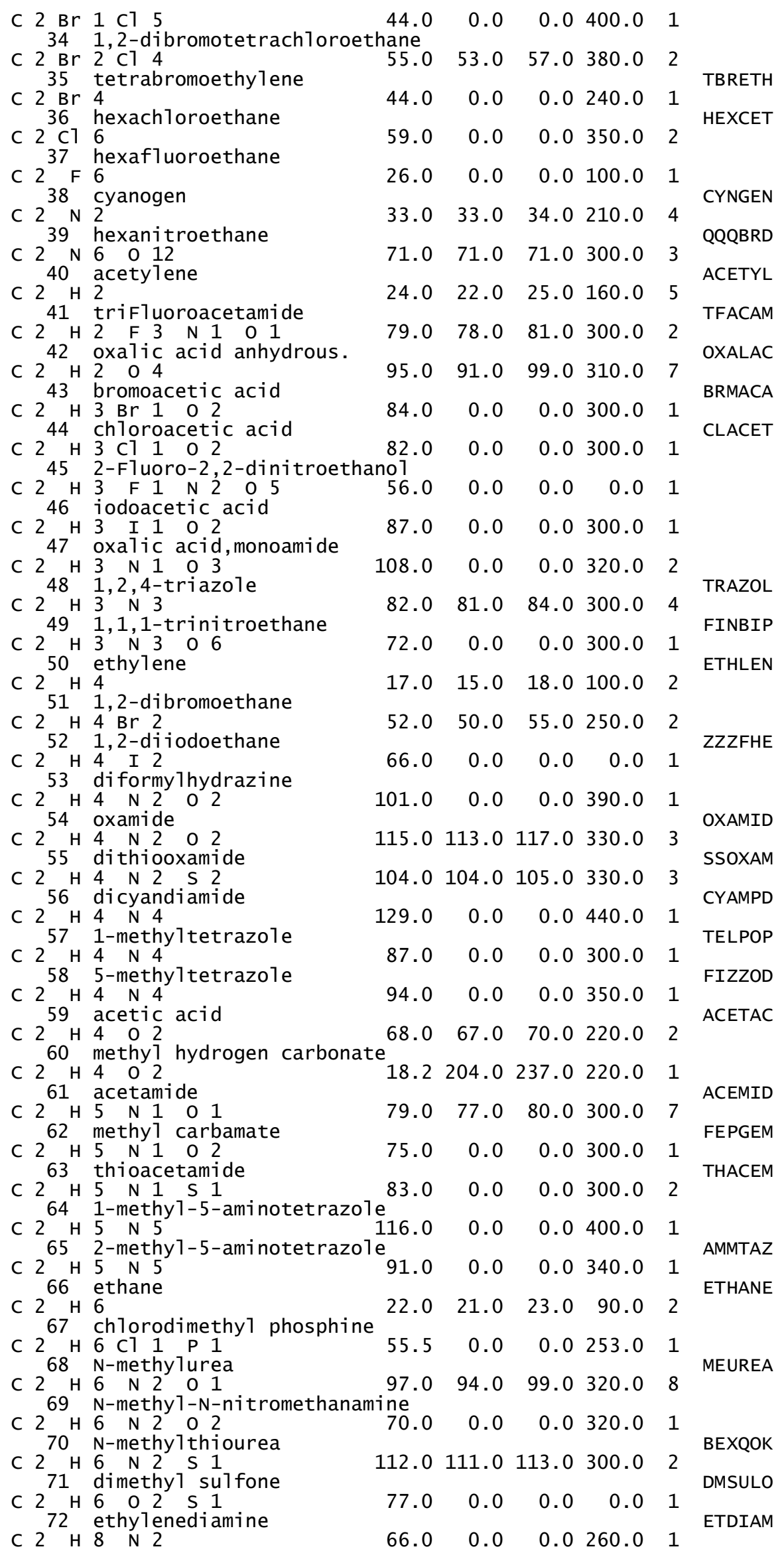




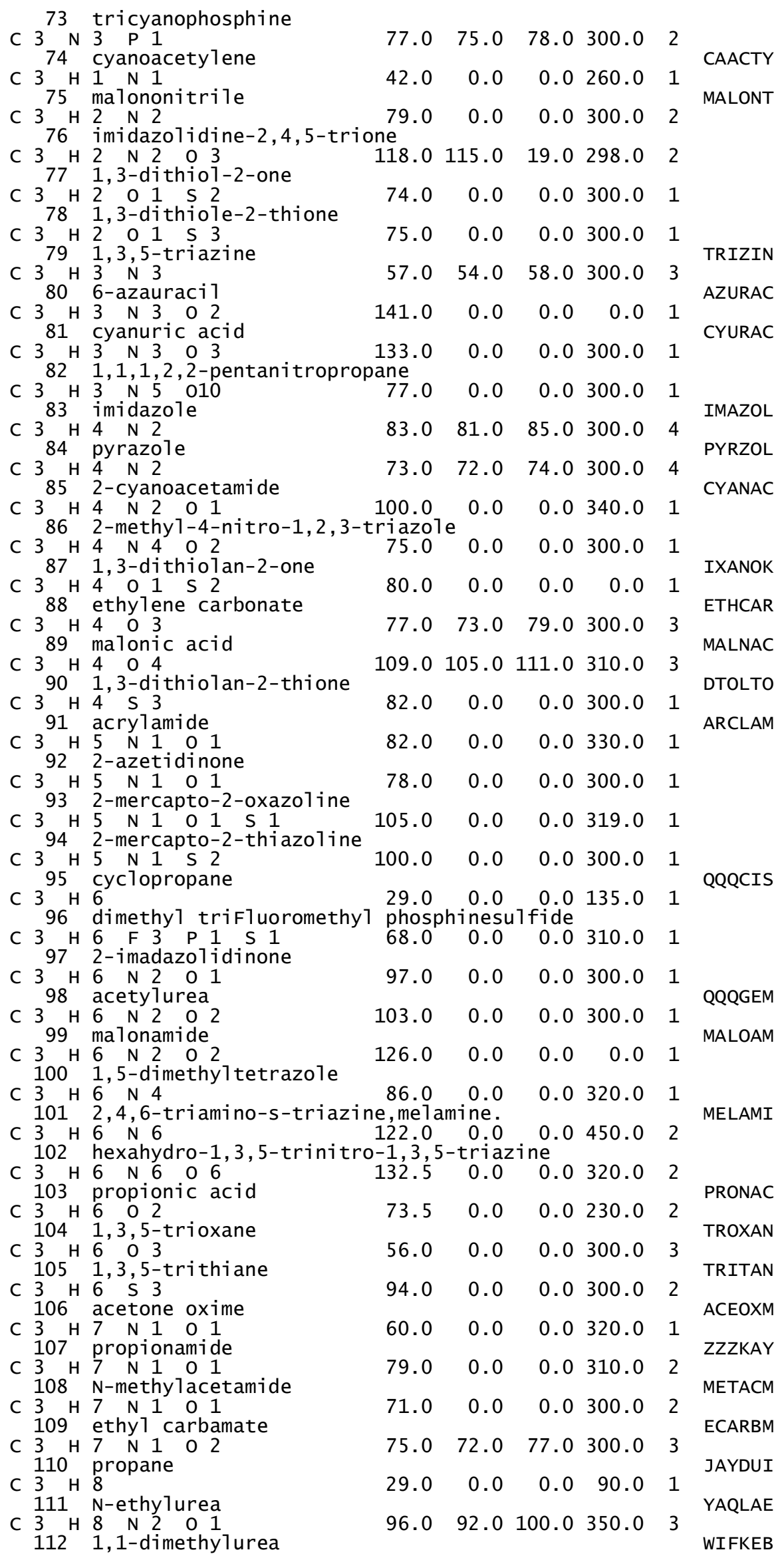




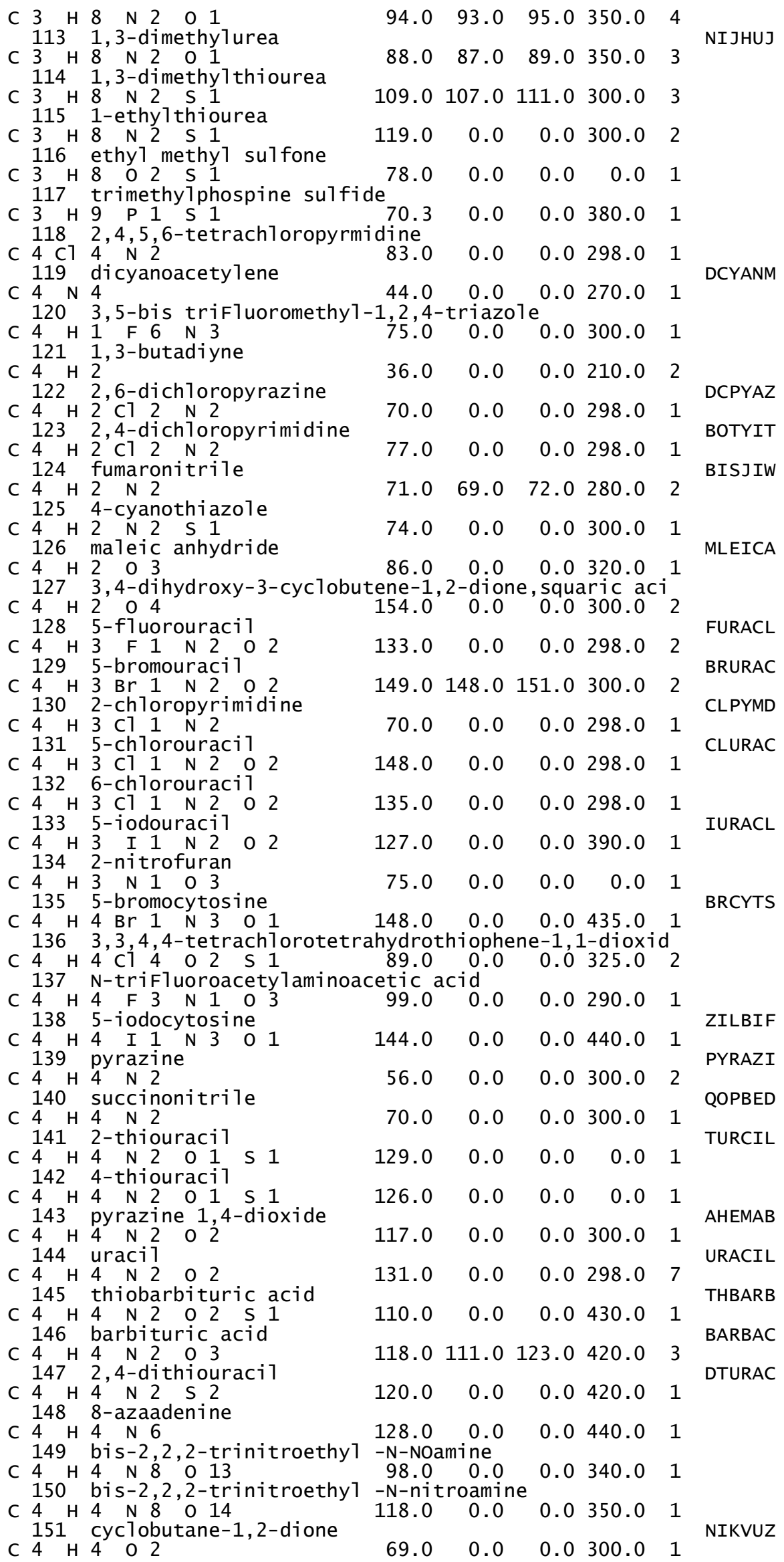




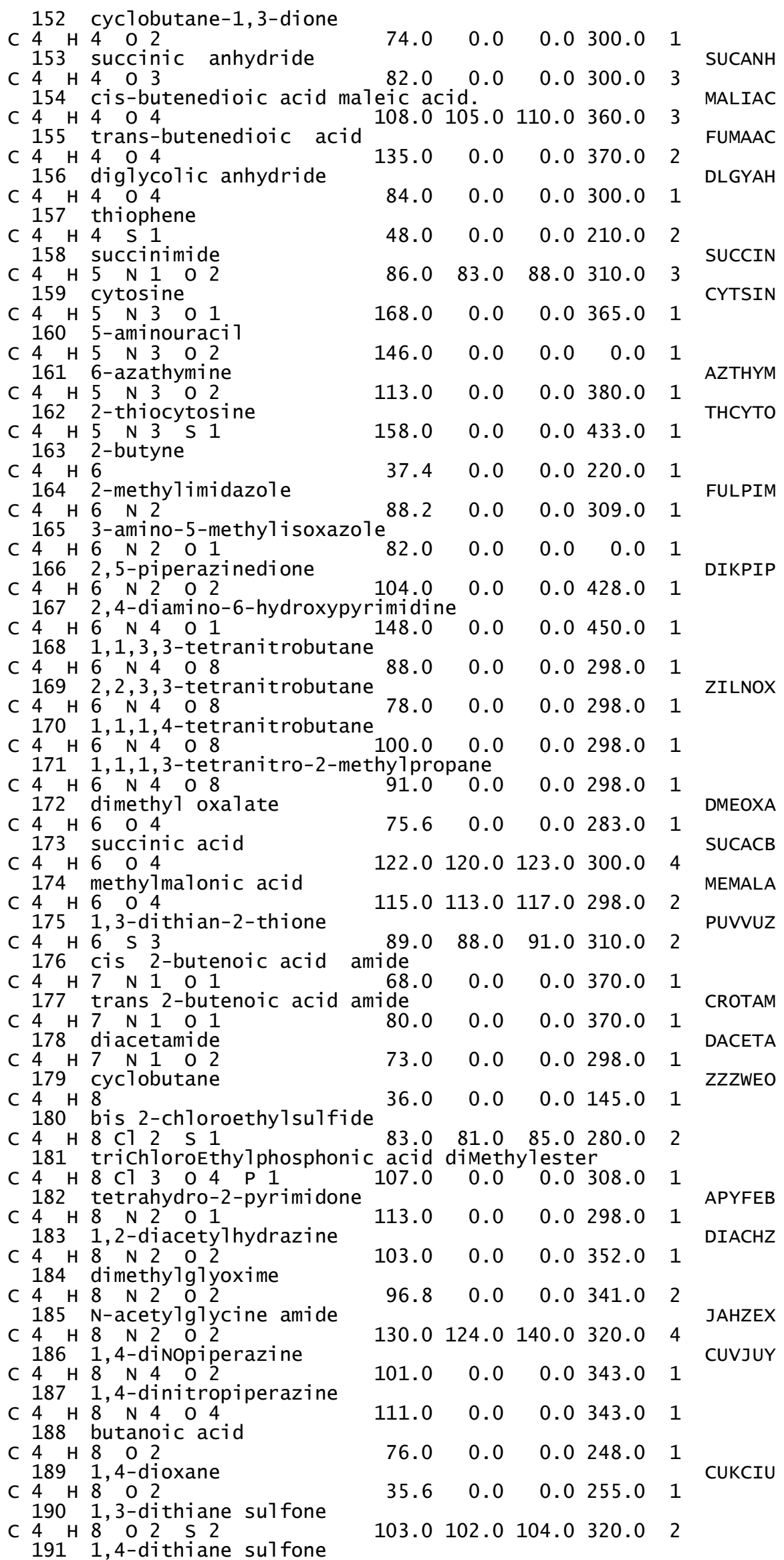




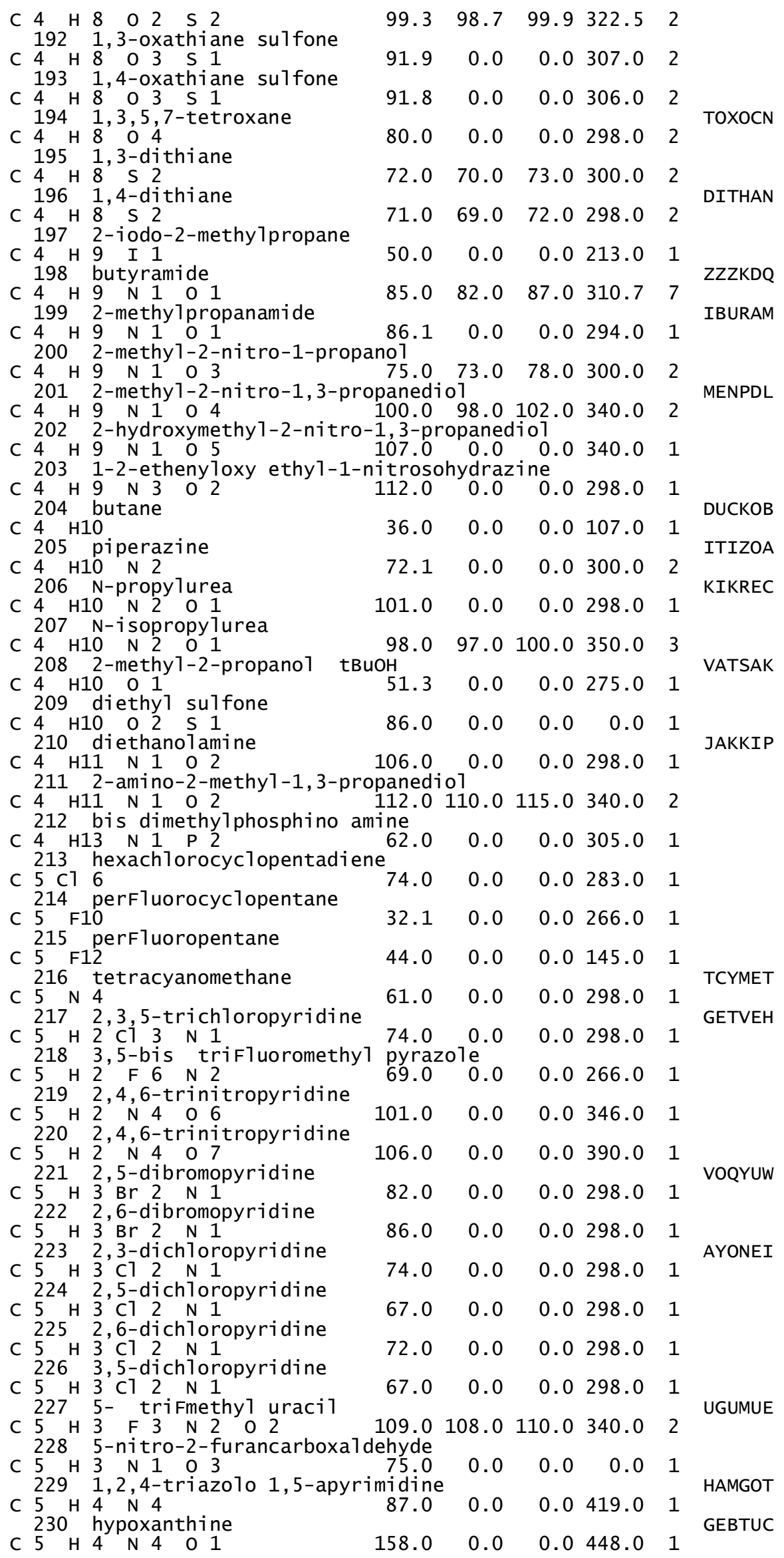




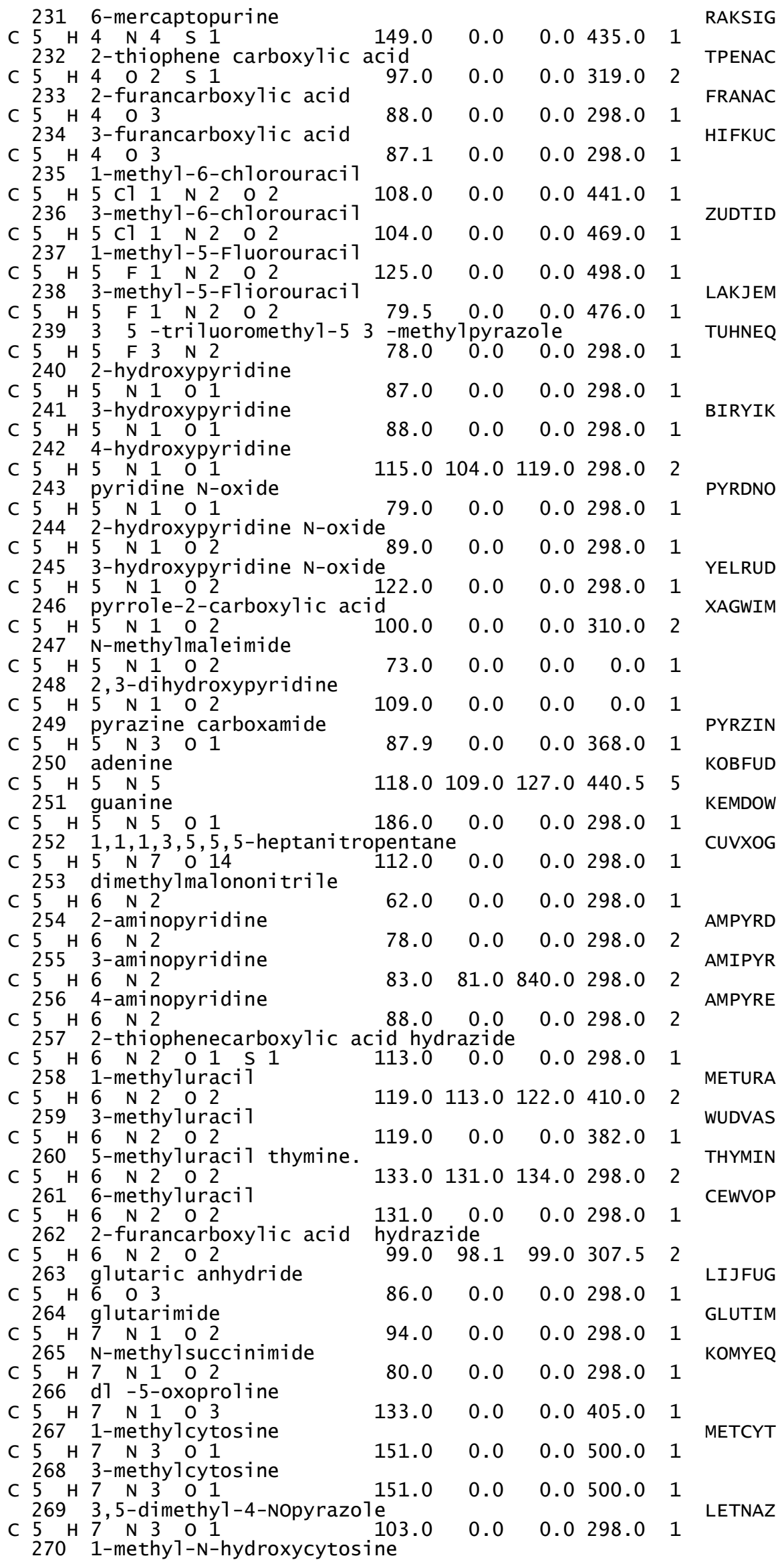




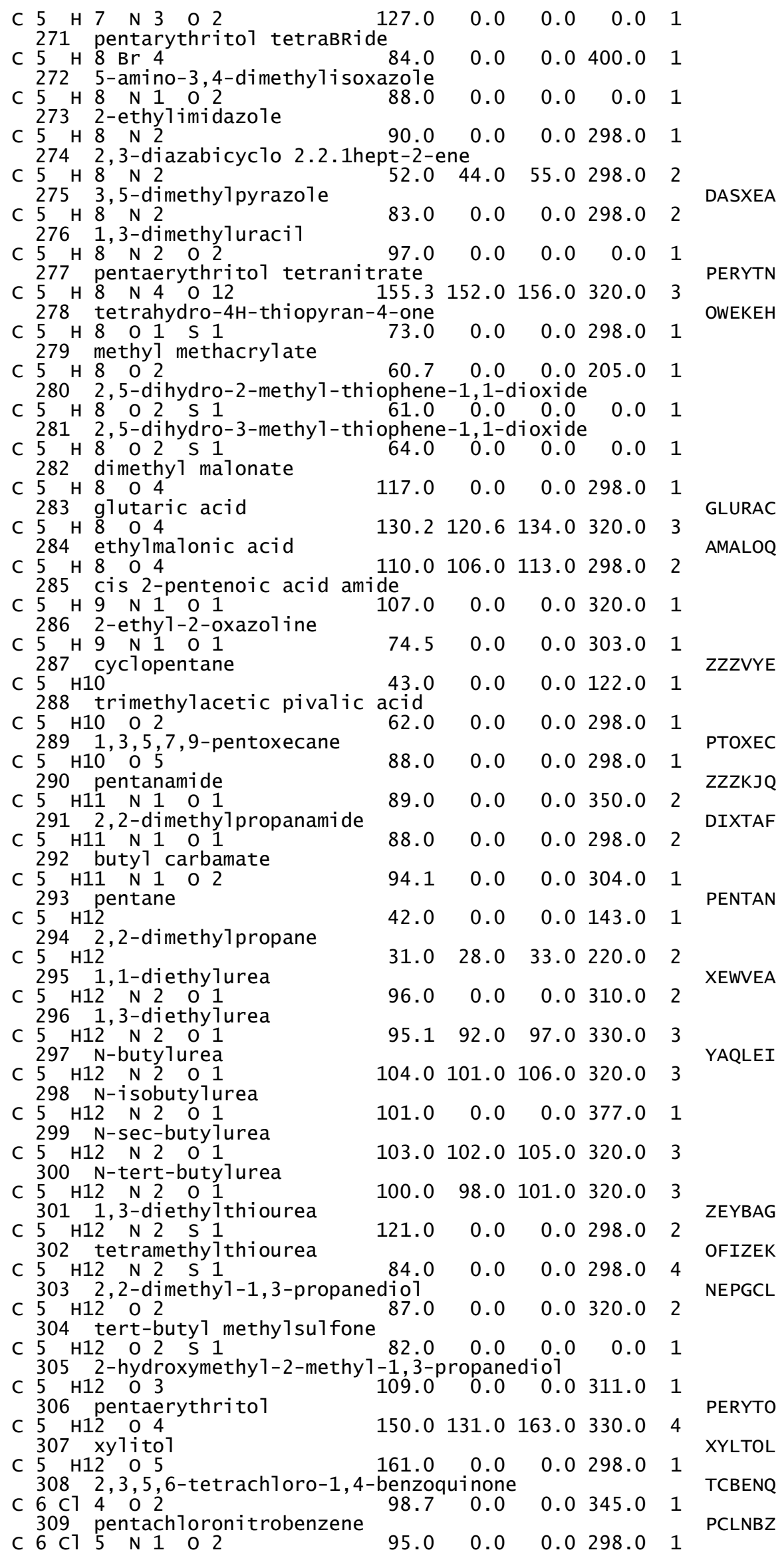




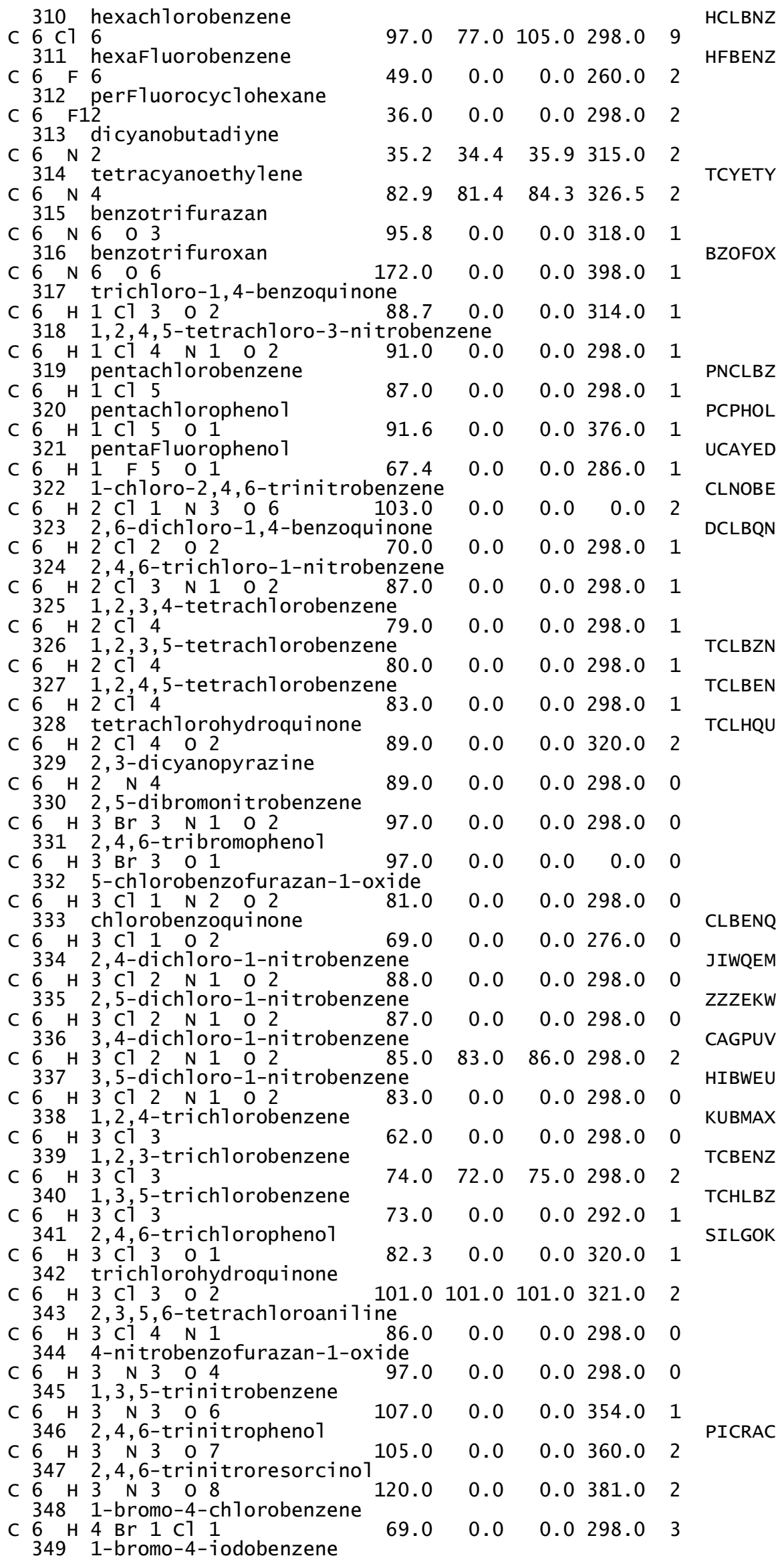




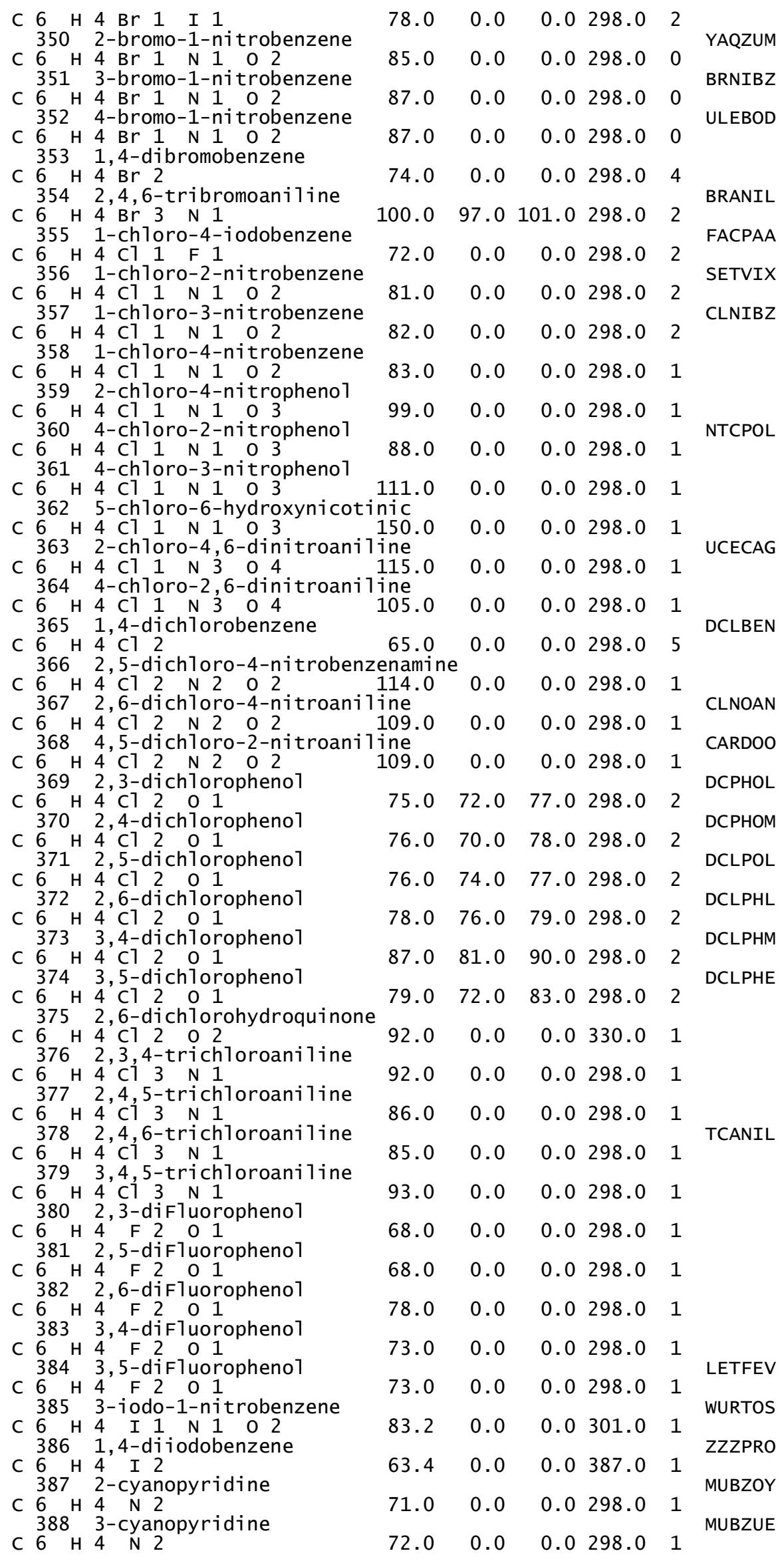




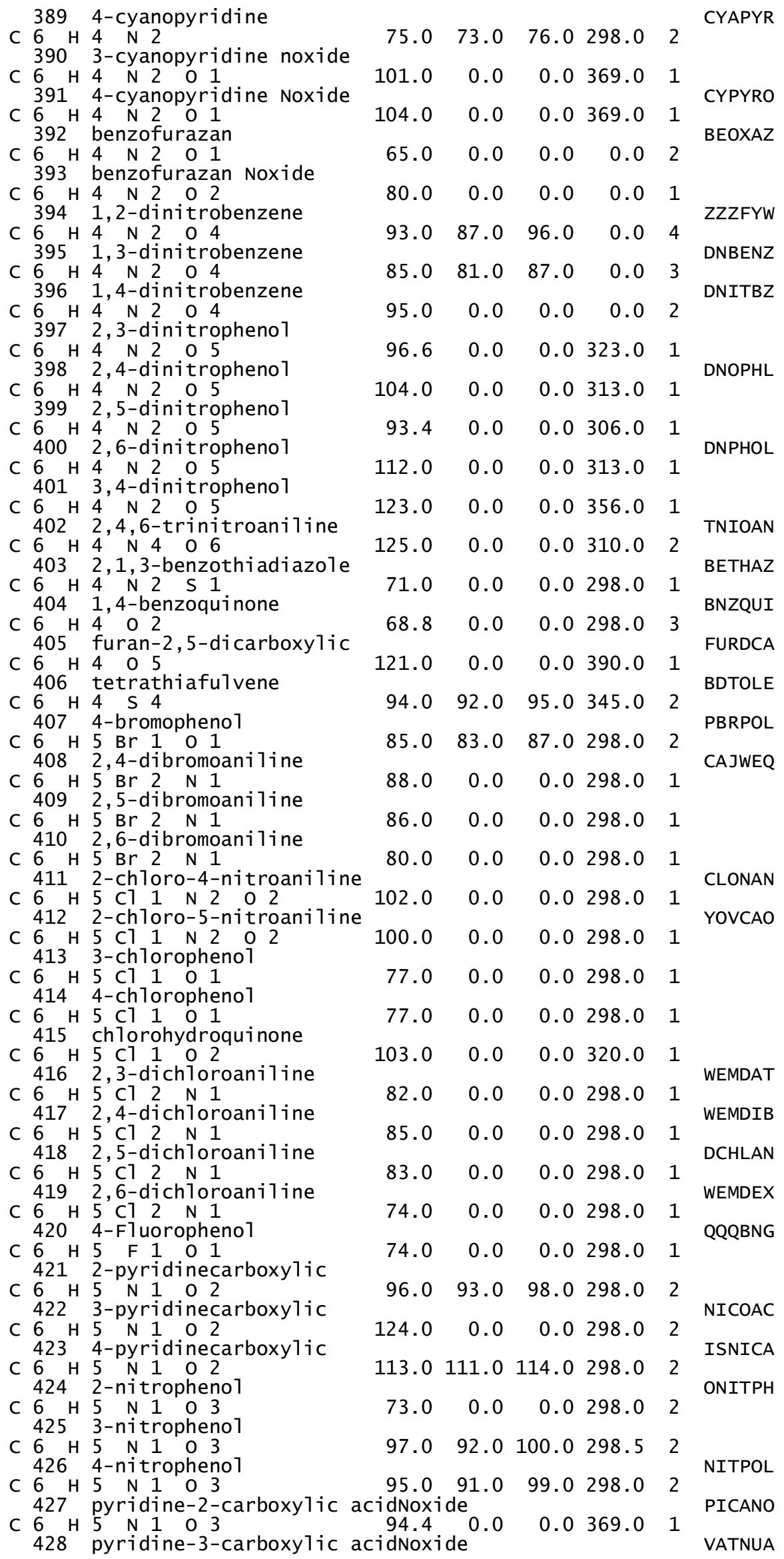




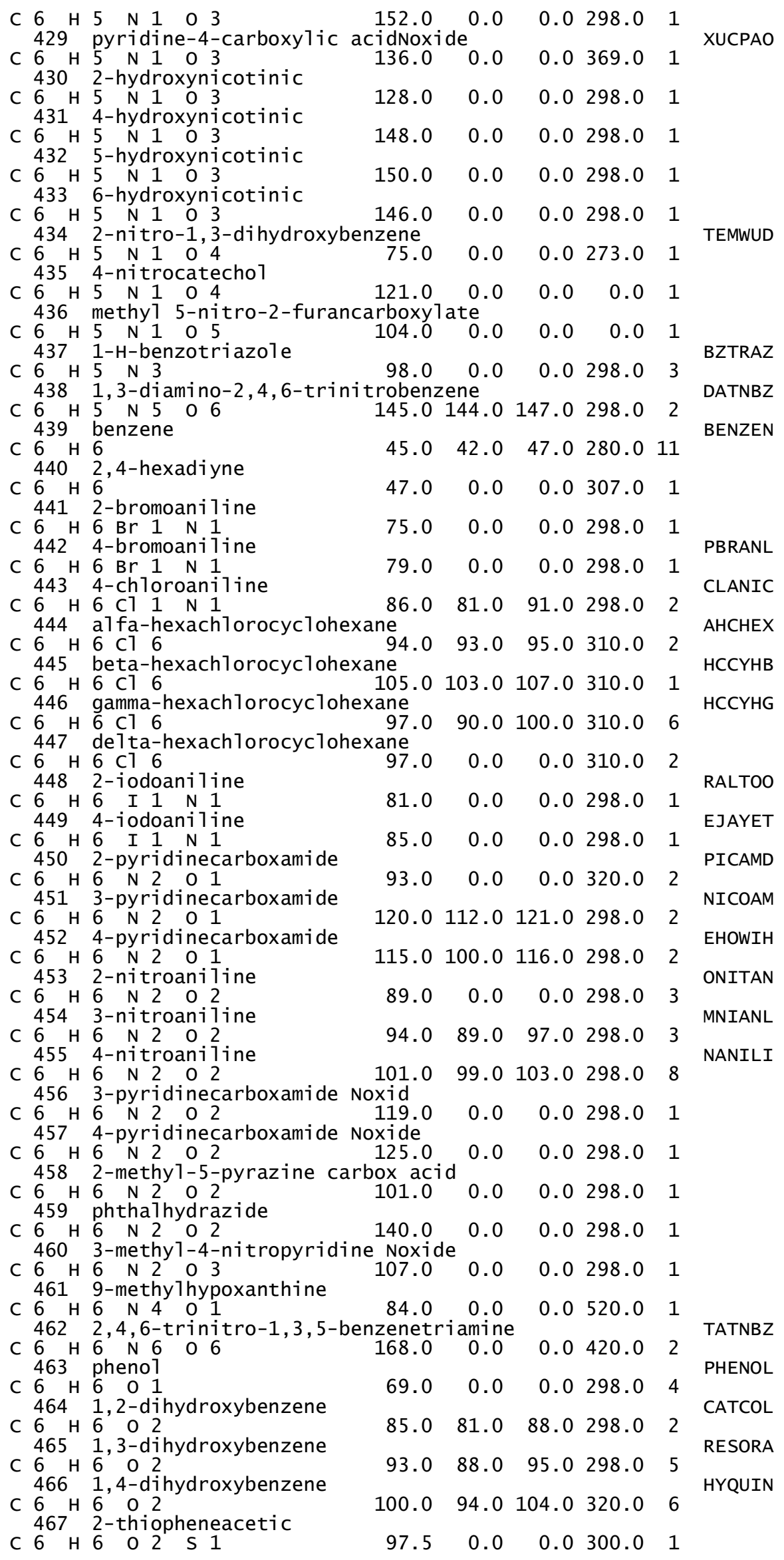




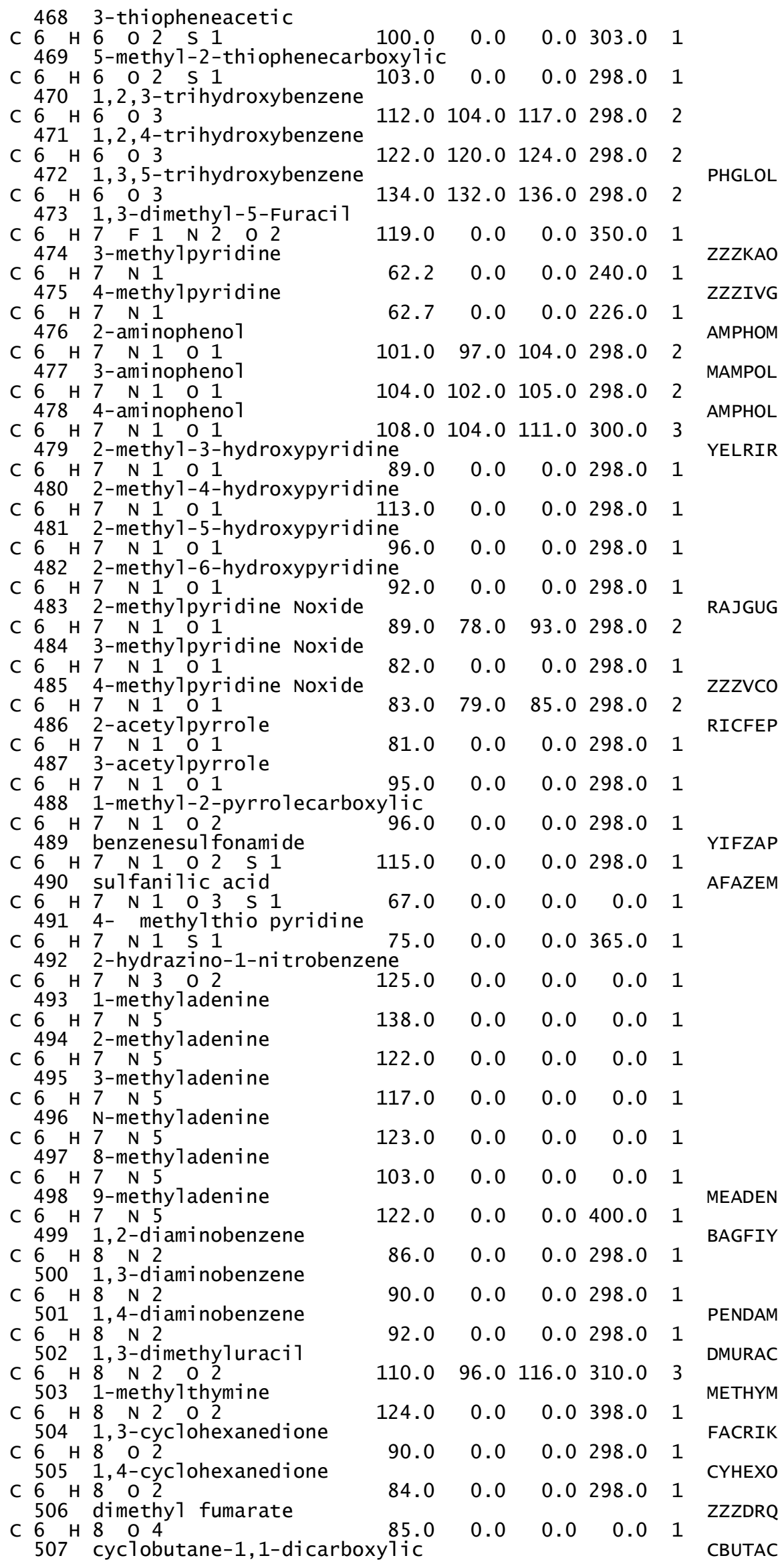




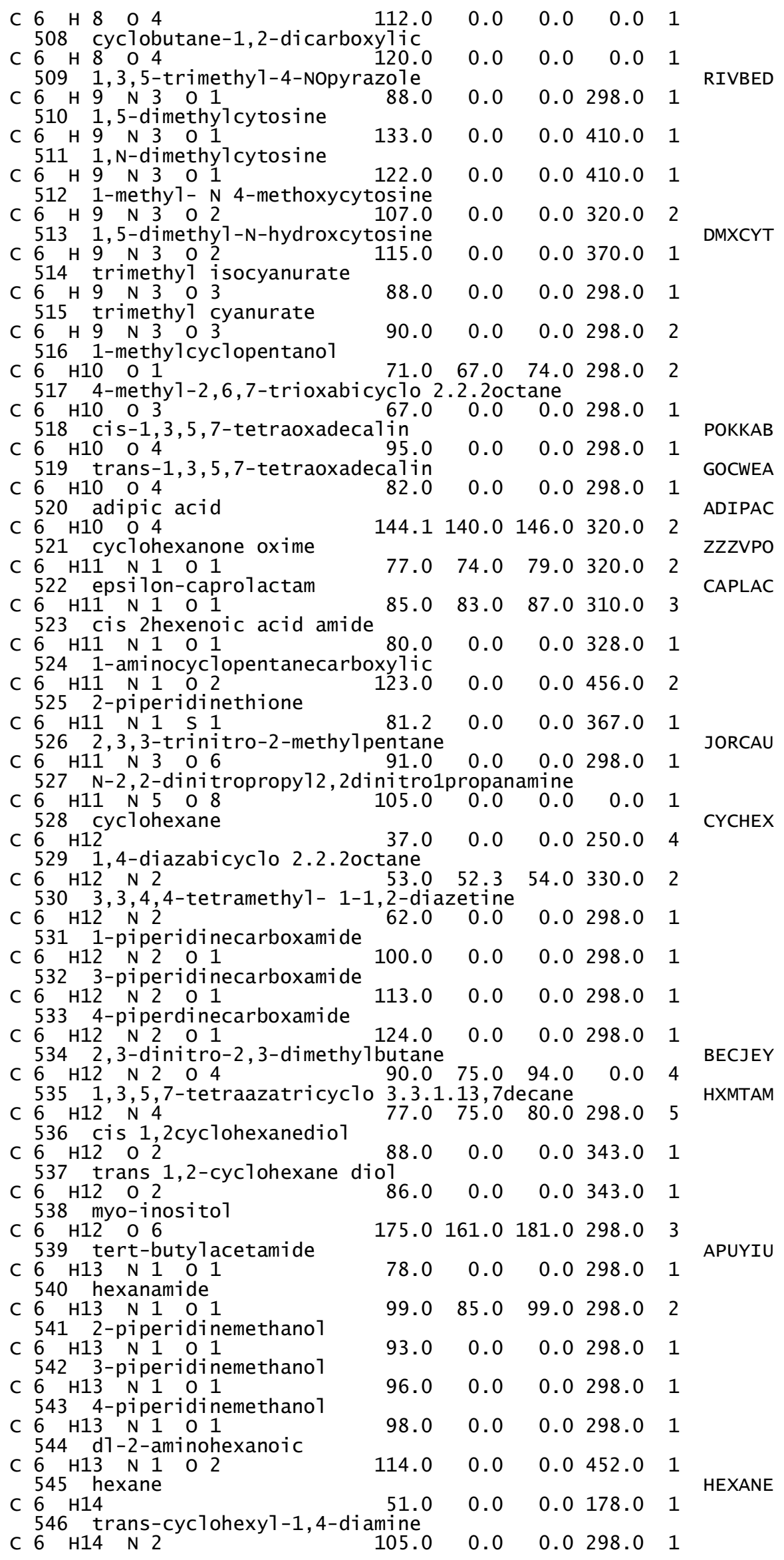


547 1,6-hexanediol

C $6 \mathrm{H} 14$ O 2

$110.0 \quad 109.0 \quad 112.0 \quad 298.0 \quad 2$

FECCOF

548 tert-buty1 ethyl sulfone

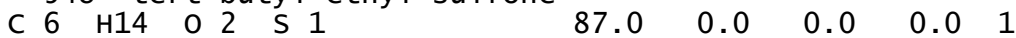

$549 \mathrm{~N}, \mathrm{~N}$-bis 2-hydroxyethyl ethylenediamine

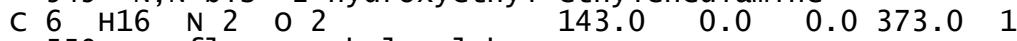
550 perfluoromethylcyclohexane

$\begin{array}{lllllll}\text { C } 7 \mathrm{~F} 14 & 52.0 & 0.0 & 0.0 & 234.0 & 1\end{array}$

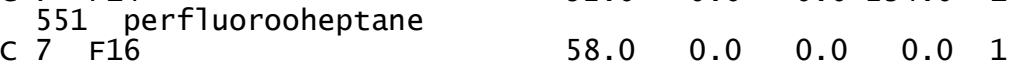

$\begin{array}{lllllll}552 & \text { pentafluorobenzoic acid } & & & & & \\ 7 & & \end{array}$

553 2-nitrobenzonitrile

C $7 \mathrm{H}_{554}$ 3 N 2 -nitrobenzonitrile

C 7 H 4 N 202

$\begin{array}{lllll}88.0 & 0.0 & 0.0 & 298.0 & 1\end{array}$

555 4-nitrobenzonitrile

C $7 \mathrm{H} 4 \mathrm{~N} 2022$

556 2,4-dinitrobenzoic acid

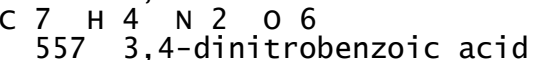

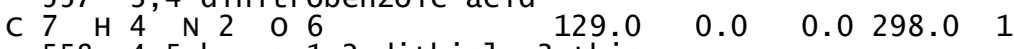

558 4,5-benzo-1,2-dithiole-3-thione

C $7 \mathrm{H} 4 \mathrm{~S} 3 \quad 107.0 \quad 0.0 \quad 0.0298 .0 \quad 1$

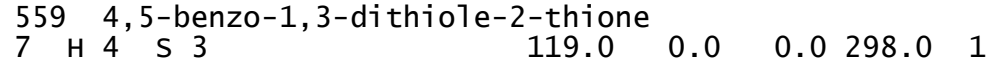

560 2-bromobenzoic acid

C 7 H 5 Br 1 O 2

561 3-bromobenzoic acid

C 7 H 5 Br 102

562 4-bromobenzoic acid

C 7 H 5 Br 1 O 2

563 2-chlorobenzoic acid

C $\begin{array}{llllll}7 & \text { H } & 5 & \mathrm{Cl} & 1 & 0\end{array}$

564 3-chlorobenzoic acid

C $\begin{array}{lllllllll} & \text { H } & 5 & \text { Cl } & 1 & 0 & 2\end{array}$

565 4-chlorobenzoic acid

C 7 H 5 Cl 102

566 2-fluorobenzoic acid

C 7 H 5 F 1 O 2

$\begin{array}{rrrrr}119.0 & 0.0 & 0.0 & 298.0 & 1 \\ 109.0 & 95.0 & 111.0 & 298.0 & 3\end{array}$

567 3-fluorobenzoic acid

C 7 H 5 F 1 o 2 -fluorobenzoic acid

C $\begin{array}{llllll}7 & \text { H } & 5 & \text { F } 1 & 0 & 2\end{array}$

569 trifluoromethylbenzene

C 7 H 5 F 3

570 2-iodobenzoic acid

C 7 H 5 I 1 O 2

571 3-iodobenzoic acid

C 7 H 5 I 1 O 2

572 4-iodobenzoic acid

C 7 H 5 I 1 O 2

$105.0 \quad 99.0 \quad 106.0 \quad 298.0 \quad 3$

$107.0103 .0 \quad 110.0 \quad 298.0 \quad 3$

$110.0106 .0 \quad 116.0 \quad 298.0 \quad 3$

$104.0101 .0106 .0 \quad 298.0 \quad 4$

$105.0103 .0 \quad 107.0 \quad 298.0 \quad 3$

PFBZAC

573 benzoxazole

C 7 H 5 N 1 O 1

574 2-benzoxazolinone

C 7 H 5 N 1 O 2

$\begin{array}{llllll}94.0 & 0.0 & 0.0 & 298.0 & 1\end{array}$

$\begin{array}{lllll}94.3 & 0.0 & 0.0 & 298.0 & 1\end{array}$

$\begin{array}{lllll}93.0 & 0.0 & 0.0 & 298.0 & 1\end{array}$

$\begin{array}{llllll}55.0 & 0.0 & 0.0 & 227.0 & 1\end{array}$

$111.0 \quad 93.0 \quad 113.0 \quad 298.0 \quad 3$

$109.0 \quad 97.0 \quad 111.0 \quad 298.0 \quad 2$

$110.0 \quad 99.0 \quad 111.0 \quad 298.0 \quad 2$

BRBZAC

MBBNZA

BRBZAP

CLBZAC

MCBZAC

CLBZAP

FBENZA

COVJIG

PFBZAD

XOGJAG

OIBZAC

ZZZOAE

BENMOW

$\begin{array}{lllll}575 & 1,1-d i o x o-1,2-b e n z i s o t h i a z o i-3 & 0.0 & 0.0298 .0 & 1\end{array}$

$\begin{array}{lllllllllll}\mathrm{C} & 7 \mathrm{H} 5 & \mathrm{~N} 1 & 0 & 3 & \mathrm{~S} 1 & 113.0 & 0.0 & 0.0 & 298.0 & 1\end{array}$

576 2-nitrobenzoic acid

C 7 H 5 N 1 O 4

577 3-nitrobenzoic acid

C 7 H $\begin{array}{lll}5 & \mathrm{~N} 1 & 0 \\ 578 & 4-\text { nitrobenzoic acid }\end{array}$

$119.0 \quad 0.0 \quad 0.0 \quad 298.0 \quad 1$

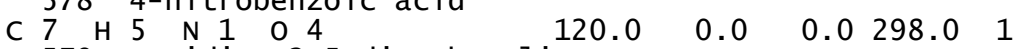
579 pyridine-2,5-dicarboxylic

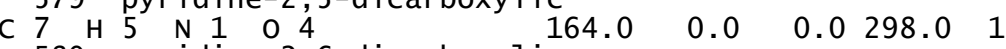

$\begin{array}{llllll}580 & \text { pyridine-2,6-dicarboxylic } & & & & \\ 7 & 137.0 & 0.0 & 0.0 & 298.0 & 1\end{array}$

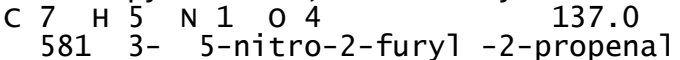

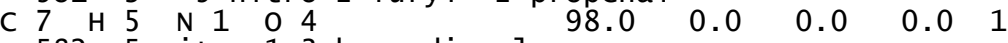
582 5-nitro-1,3-benzodioxole

C 7 H 5 N 1 O 4

$\begin{array}{lllll}97.0 & 0.0 & 0.0 & 298.0 \quad 1\end{array}$

583 benzothiazole

C 7 H 5 N 1 S 1

$5842,4,6$-trinitrotoluene

C $7 \mathrm{H} 5 \mathrm{~N} 3$ o 6

585 2,4,6-trinitroanisole

$\begin{array}{lllllllll}\mathrm{C} 7 \mathrm{H} 5 & \mathrm{~N} 3 & 0 & 7 & 133.0 & 0.0 & 0.0 & 330.0 & 2\end{array}$

$\begin{array}{lllll}73.0 & 0.0 & 0.0 & 298.0 \quad 1\end{array}$

$110.0 \quad 92.0 \quad 118.0 \quad 298.0 \quad 7$

ZZZMUC

NBZOAO

MNBZAC

NBZOAC

AFEBUI

586 3-hydroxy-2, 4,6-trinitrotoluene 


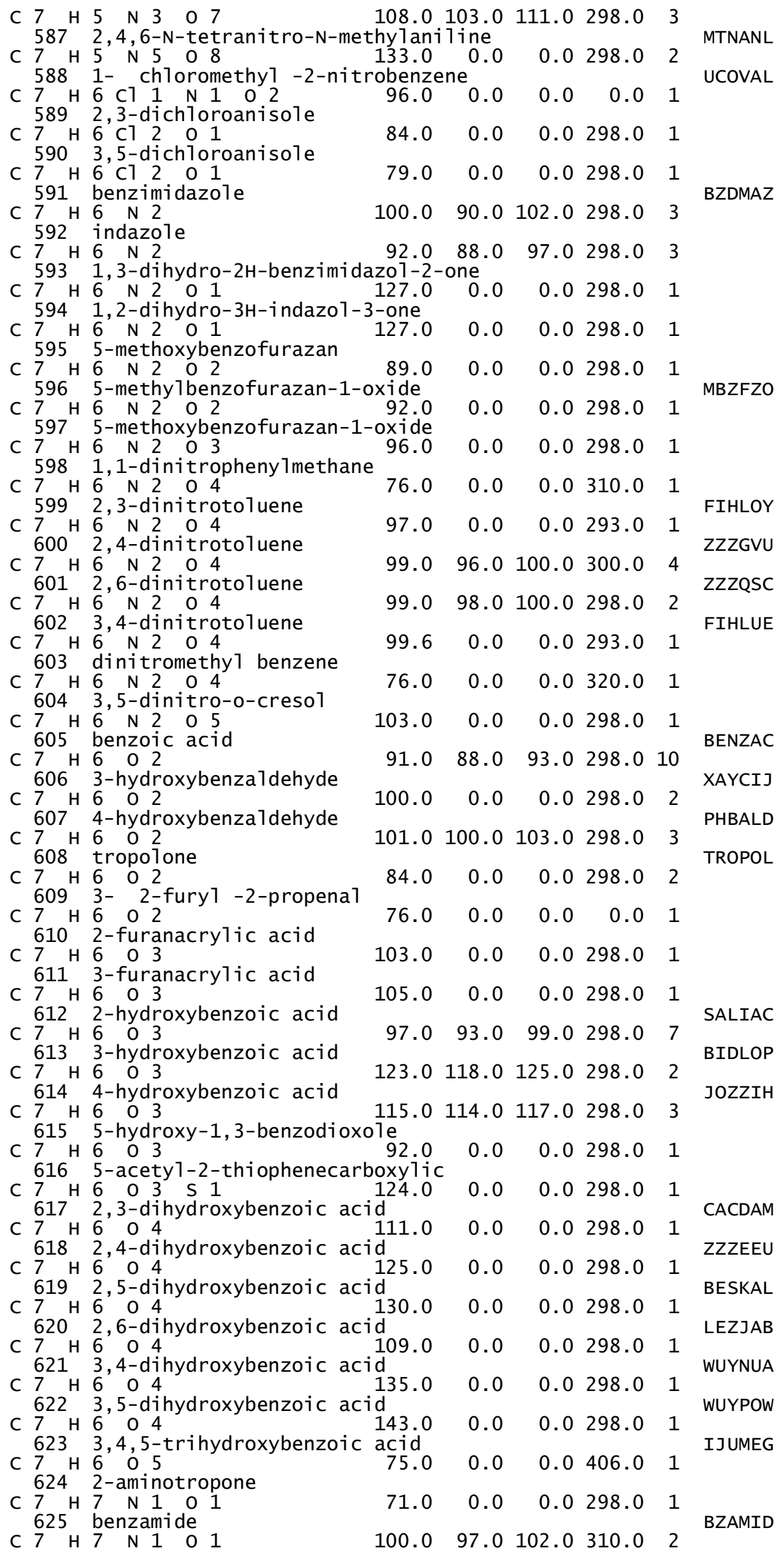




\begin{tabular}{|c|c|c|c|c|c|c|c|}
\hline C ${ }_{7}^{626} \mathrm{H}$ & $\begin{array}{l}\text { formanilide } \\
7 \mathrm{~N} 1 \mathrm{O}\end{array}$ & 78.0 & 0.0 & 0.0 & 308.0 & 1 & \\
\hline C $7 \mathrm{H}^{\mathrm{H}}$ & $7 \times 1002$ & 77.0 & 75.0 & 79.0 & 298.0 & 2 & NITOLU \\
\hline $7^{628} \mathrm{H}$ & $\begin{array}{l}2 \text {-aminobenzoic acid } \\
7 \times 1 \text { o } 2\end{array}$ & 110.0 & 105.0 & 112.0 & 298.0 & 2 & AMBACO \\
\hline${ }_{7}^{629} \mathrm{H}$ & $\begin{array}{l}3 \text {-aminobenzoic acid } \\
7 \mathrm{~N} 1 \text { o } 2\end{array}$ & 128.0 & 0.0 & 0.0 & 298.0 & 1 & AMBNZA \\
\hline 630 & $\begin{array}{l}4 \text {-aminobenzoic acid } \\
7 \mathrm{~N} 1 \text { o } 2\end{array}$ & 116.0 & 0.0 & 0.0 & 298.0 & 1 & AMBNAC \\
\hline $7^{631} \mathrm{H}$ & $\begin{array}{l}\text { 2-hydroxybenzamide } \\
7 \quad \mathrm{~N} 1 \\
\begin{array}{llll}1 & 0\end{array}\end{array}$ & 101.0 & 99.0 & 102.0 & 298.0 & 2 & SALMID \\
\hline $7^{632} \mathrm{H}$ & $\begin{array}{l}\text { 4-hydroxybenzami de } \\
7 \quad \mathrm{~N} 1 \\
1\end{array}$ & 125.0 & 118.0 & 130.0 & 298.0 & 2 & VIDMAX \\
\hline $7 \mathrm{H}$ & $7 \mathrm{~N} 1$ o 4 & 99.0 & 0.0 & 0.0 & 298.0 & 1 & \\
\hline C $7635 \mathrm{H}$ & 7 N 1 o 4 & 106.0 & 0.0 & 0.0 & 298.0 & 1 & \\
\hline $636^{7}$ & $7 \mathrm{~N} 1$ o 4 & 91.0 & 0.0 & 0.0 & 298.0 & 1 & \\
\hline C ${ }_{637} \mathrm{H}$ & $\begin{array}{l}7 \mathrm{~N} 1 \text { S } 1 \\
\text { toluene }\end{array}$ & 100.0 & 97.0 & 103.0 & 298.0 & 2 & ZUNSEU \\
\hline $638^{7}$ & $\begin{array}{l}8 \\
1 \text {-amino-7-imino- } 1,3,5-c)\end{array}$ & $\begin{array}{l}43.0 \\
\text { yclohep }\end{array}$ & $\begin{array}{l}0.0 \\
\text { tatrie }\end{array}$ & & 298.0 & 1 & \\
\hline $639^{7}$ & $\begin{array}{l}8 \mathrm{~N} 2 \\
\text { monophenylurea }\end{array}$ & 49.4 & 0.0 & 0.0 & 303.0 & 1 & PHUREA \\
\hline C $7640 \mathrm{H}$ & $\begin{array}{l}8 \text { N } 2 \text { o } 1 \\
\text { 2-pyridy } 1 \text { acetamide }\end{array}$ & 136.0 & 0.0 & 0.0 & 406.0 & 1 & \\
\hline C ${ }_{641}^{7} \mathrm{H}$ & $\begin{array}{l}8 \mathrm{~N} 201 \\
9 \text {-Ethylhypoxanthine }\end{array}$ & 104.0 & 0.0 & 0.0 & 298.0 & 1 & \\
\hline C $7{ }_{642}{ }^{H}$ & $\begin{array}{l}8 \mathrm{~N} 4 \text { o } 1 \\
1,9 \text {-dimethylhypoxanthine }\end{array}$ & $e^{108.0}$ & 0.0 & 0.0 & 0.0 & 1 & \\
\hline $7_{643}{ }^{H}$ & $\begin{array}{l}8 \mathrm{~N} 4 \text { o } 1 \\
1,3 \text {-dimethy } 1 \text { xanthine the }\end{array}$ & $\begin{array}{c}75.0 \\
\text { eophy } 11\end{array}$ & line 0.0 & 0.0 & 0.0 & 1 & \\
\hline$={ }^{7} 4^{\mathrm{H}}$ & $\begin{array}{l}8 \text { N } 4 \text { o } 2 \\
\text { 2-hydroxytoluene }\end{array}$ & $140 \Omega$ & 135.0 & 144.0 & 298.0 & 3 & OCRSOL \\
\hline C $7{ }_{645} \mathrm{H}$ & $\begin{array}{l}801 \\
3 \text {-hydroxytoluene }\end{array}$ & 75.4 & 74.8 & 76.0 & 288.0 & 3 & \\
\hline C $7{ }_{646}^{H}$ & $\begin{array}{l}8001 \\
4 \text {-hydroxytoluene }\end{array}$ & 60.0 & 56.0 & 62.0 & 298.0 & 2 & CRESOI \\
\hline C $7_{647}{ }^{\mathrm{H}}$ & $\begin{array}{l}81 \\
3 \text {-methy } 1-1,2 \text {-dihydroxybe }\end{array}$ & $\begin{array}{r}74.0 \\
\text { enzene }\end{array}$ & 0.0 & 0.0 & 298.0 & 2 & CTLUUL \\
\hline C ${ }_{648}{ }^{\mathrm{H}}$ & $\begin{array}{l}80 \\
2,4-d i h y d r o x y t o l u e n e\end{array}$ & 93.0 & 0.0 & 0.0 & 298.0 & + & \\
\hline C ${ }_{649}{ }^{H}$ & $\begin{array}{l}8 \\
2,6 \text {-dihydroxytoluene }\end{array}$ & 107.0 & 0.0 & 0.0 & 298.0 & 1 & NUOKER \\
\hline C $7{ }_{650} \mathrm{H}$ & $\begin{array}{l}802 \\
3,4-d i h y d r o x y t o l u e n e\end{array}$ & 99.0 & 0.0 & 0.0 & 298.0 & 1 & \\
\hline$={ }_{651}^{7} \mathrm{H}$ & $\begin{array}{l}802 \\
3,5 \text {-dihydroxytoluene }\end{array}$ & 95.0 & 0.0 & 0.0 & 298.0 & 1 & EWAMAR \\
\hline C $7652^{\mathrm{H}}$ & 2-methy $1-1,4$-dihydroxybe & $\begin{array}{l}103.0 \\
\text { enzene }\end{array}$ & 0.0 & 0.0 & 298.0 & 1 & 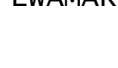 \\
\hline${ }^{7} 653^{H}$ & $\begin{array}{l}802 \\
\text { 4-methoxyphenol }\end{array}$ & 105.0 & 100.0 & 105.0 & 298.0 & 2 & \\
\hline C $7{ }_{654}^{H}$ & $\begin{array}{l}80 \text { o } 2 \\
\text { MEphenylsulfone }\end{array}$ & 92.0 & 89.0 & 94.0 & 298.0 & 2 & MPSUFO \\
\hline $7_{655} \mathrm{H}$ & $\begin{array}{l}802 \mathrm{~S} 1 \\
4,5 \text {-tetramethylene- } 1,3\end{array}$ & $\begin{aligned} 92.0 \\
\text { dithiol }\end{aligned}$ & $\begin{array}{l}0.0 \\
\text { le-2-th }\end{array}$ & $\begin{array}{l}0.0 \\
\text { hione }\end{array}$ & 0.0 & 1 & 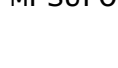 \\
\hline C $7_{656} \mathrm{H}$ & $\begin{array}{l}8 \mathrm{~S} 3 \\
4,5 \text {-tetramethylene }-1,2-c\end{array}$ & dithiol & $\begin{array}{c}0.0 \\
\text { e-3-th }\end{array}$ & $\begin{array}{l}0.0 \\
\text { hione }\end{array}$ & 298.0 & 1 & \\
\hline C $7_{657^{H}}^{\mathrm{H}}$ & $\begin{array}{l}8 \mathrm{~S} 3 \\
\text { p-toluidine }\end{array}$ & 0 & 0.0 & 0.0 & 298.0 & 1 & TOLDIN \\
\hline C $7{ }_{658}^{H}$ & $\begin{array}{l}9 \mathrm{~N} 1 \\
3,5-\text { dimethy } 1 \text { pyridine Nox }\end{array}$ & $\begin{array}{l}78.0 \\
\text { xide }\end{array}$ & 76.0 & 79.0 & 298.0 & 2 & \\
\hline C $7_{659}{ }^{H}$ & $\begin{array}{l}9 \text { N } 1 \text { o } 1 \\
8,9 \text {-dimethyladenine }\end{array}$ & & 0.0 & 0.0 & 298.0 & $\perp$ & \\
\hline $\begin{array}{l}7 \\
660^{H}\end{array}$ & $\begin{array}{l}9 \times 5 \\
2,9-\text { dimethyladenine }\end{array}$ & 105.0 & 0.0 & 0.0 & 350.0 & 1 & \\
\hline${ }_{661} \mathrm{H}$ & $9 \mathrm{~N} 5$ & 123.0 & 0.0 & 0.0 & 350.0 & 1 & \\
\hline C $7_{662} \mathrm{H}$ & $\begin{array}{l}9 \times 5 \\
N, 9-\text { dimethyladenine }\end{array}$ & 116.0 & 0.0 & 0.0 & 350.0 & 1 & DIMIADEN \\
\hline C $7663^{H}$ & $\begin{array}{l}9 \text { N } 5 \\
\text { bicyclo 2.2.1hept-2-ene }\end{array}$ & 116.0 & 0.0 & 0.0 & 350.0 & 1 & \\
\hline C ${ }_{664}{ }^{H 1}$ & tricyclo 2.2.1.02,6hept & $\begin{array}{ll}37.0 \\
\text { ane }\end{array}$ & 34.0 & 39.0 & 298.0 & 4 & \\
\hline C ${ }_{665} \mathrm{H} 1$ & butv $7 m a$ lonon & $\begin{array}{l}39.0 \\
r i 7 e\end{array}$ & 0.0 & 0.0 & 298.0 & 2 & \\
\hline
\end{tabular}




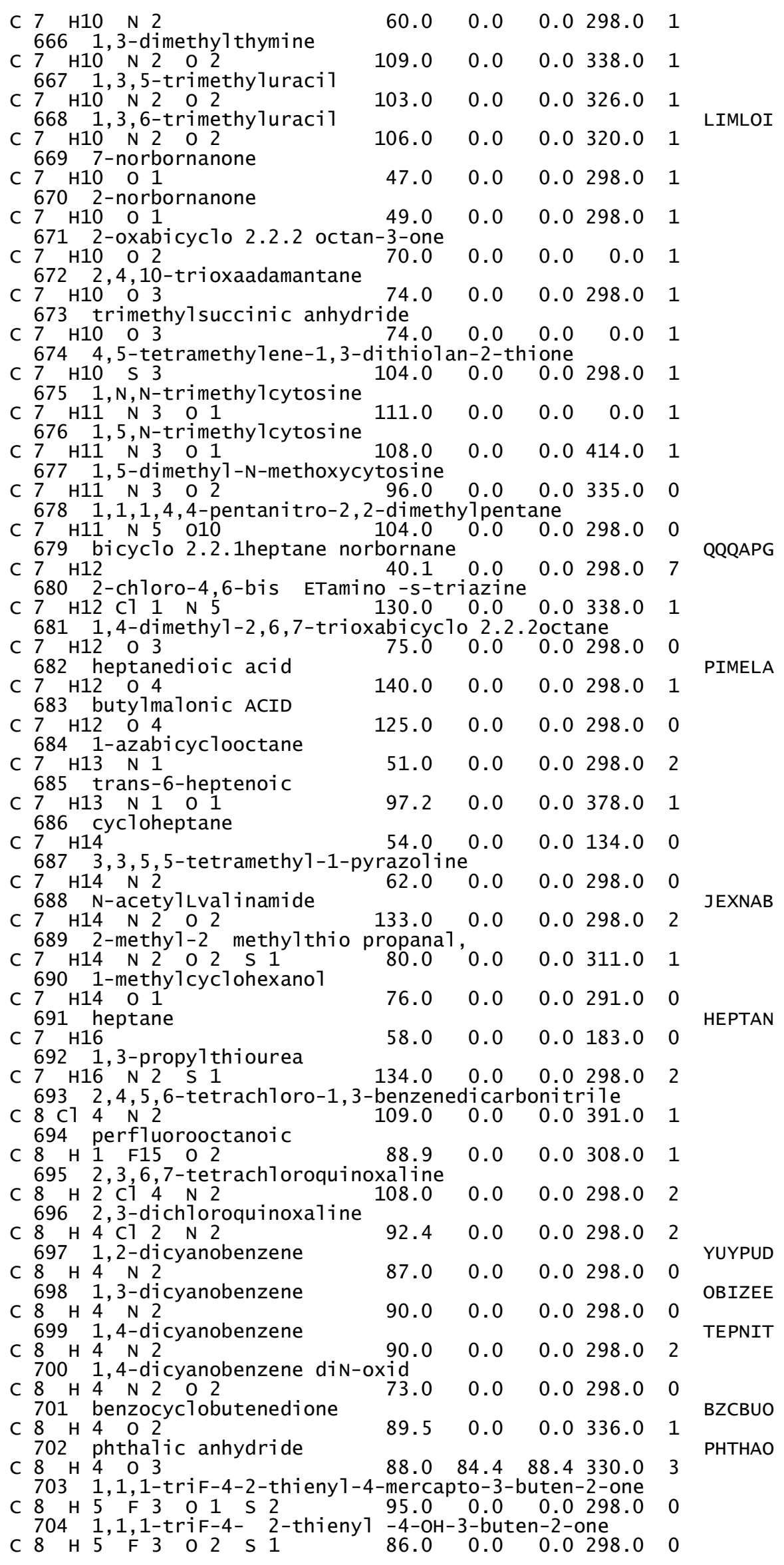




\begin{tabular}{|c|c|c|c|c|c|c|c|}
\hline 705 & $4,4,4-$ trifluoro-1- $2-\mathrm{fu}$ & uranyl & -butane- & $-1,3-$ & -dione & & \\
\hline${ }_{706}^{8}$ & $\begin{array}{l}5 \text { F } 303 \\
\text { benzoylnitrile }\end{array}$ & & & & & & \\
\hline$={ }_{707}^{\mathrm{H}}$ & $\begin{array}{l}5 \mathrm{~N} 1 \mathrm{O} 1 \\
\text { phthalimide }\end{array}$ & 78.7 & 0.0 & 0.0 & 298.0 & 1 & PHALIM \\
\hline & $\begin{array}{llll}5 & 1 & 0 & \\
1 & & \\
& & \end{array}$ & 106.0 & 0.0 & 0.0 & 298.0 & 1 & \\
\hline $8^{708} \mathrm{H}$ & 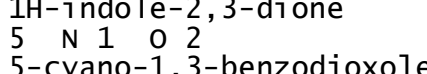 & 119.0 & 0.0 & 0.0 & 298.0 & 0 & \\
\hline $8 \mathrm{H}$ & $\begin{array}{l}3-c y a n o-1, \text { s-benzodioxole } \\
5 \text { N } 1 \text { o } 2\end{array}$ & e 91.0 & 0.0 & 0.0 & 298.0 & 0 & \\
\hline$=8_{711}^{10} \mathrm{H}$ & $\begin{array}{l}\text { 2-cyanobenzoic acid } \\
5 \mathrm{~N} 1 \mathrm{O} 2 \\
3-\text { cyanobenzoic acid }\end{array}$ & 115.0 & 0.0 & 0.0 & 298.0 & 0 & \\
\hline${ }_{712} \mathrm{H}$ & $\begin{array}{l}5 \times 1 \text { o } 2 \\
4 \text {-cvanobenzojc acid }\end{array}$ & 116.0 & 0.0 & 0.0 & 298.0 & 0 & TAGNAR \\
\hline${ }_{713}{ }^{H}$ & $\begin{array}{l}5 \mathrm{~N} 1 \\
\text { isatoic anhydride }\end{array}$ & 113.0 & 0.0 & 0.0 & 298.0 & 0 & 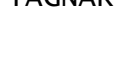 \\
\hline${ }_{714}{ }^{H}$ & $\begin{array}{l}5 \mathrm{~N} 1 \mathrm{O} 3 \\
2 \mathrm{H}-1,3 \text {-benzoxazine- } 2,4\end{array}$ & $\begin{array}{l}116.0 \\
3 \mathrm{H}-\mathrm{dic}\end{array}$ & one 0.0 & 0.0 & 298.0 & 0 & BOXAZD \\
\hline $8^{8}{ }^{10} \mathrm{H}$ & $\begin{array}{l}5 \mathrm{~N} 1 \mathrm{O} 3 \\
\text { pyridinium dicyanomethy }\end{array}$ & $\begin{array}{l}114.0 \\
\text { ide }\end{array}$ & 0.0 & 0.0 & 298.0 & 0 & \\
\hline $8^{8}{ }^{\mathrm{H}}$ & $\begin{array}{l}5 \text { N } 3 \\
2 \text {-nitrobenzeneacety } 1 \mathrm{ch} 1\end{array}$ & $\begin{array}{l}125.0 \\
\text { loride }\end{array}$ & 125.012 & 125.0 & 411.5 & 2 & \\
\hline${ }_{717}^{8} \mathrm{H}$ & $\begin{array}{l}6 \mathrm{c} 1 \mathrm{~N} 1 \mathrm{O} 3 \\
3-\mathrm{nitrobenzeneacety} 1 \mathrm{ch} 1\end{array}$ & $\begin{array}{l}104.0 \\
\text { 1oride }\end{array}$ & 0.0 & 0.0 & 311.0 & 0 & \\
\hline$={ }_{718} \mathrm{H}$ & $\begin{array}{l}6 \text { c1 } 1 \text { N } 1 \text { o } 3 \\
2,4-\text { dich1orophenoxy acet }\end{array}$ & $\begin{array}{l}109.0 \\
\text { tic }\end{array}$ & 0.0 & 0.0 & 314.0 & 0 & CPXACA \\
\hline${ }_{719}{ }^{\mathrm{H}}$ & $\begin{array}{l}6 \text { Cl } 2 \text { o } 3 \\
\text { phthalazine }\end{array}$ & 120.0 & 115.01 & 125.0 & 298.0 & 3 & DAZNAP \\
\hline$=8{ }_{720} \mathrm{H}$ & $\begin{array}{l}6 \mathrm{~N} 2 \\
\text { quinoxaline } 1,4\end{array}$ & 82.0 & 0.0 & 0.0 & 298.0 & 2 & \\
\hline${ }_{721}^{8} \mathrm{H}$ & $\begin{array}{l}6 \text { N } 2 \\
\text { quinazoline }\end{array}$ & 68.0 & 0.0 & 0.0 & 298.0 & 2 & ח ח \\
\hline C ${ }_{722}{ }^{H}$ & $\begin{array}{l}6 \text { N } 2 \\
2 \text {-hydroxyquinoxaline }\end{array}$ & 77.0 & 0.0 & 0.0 & 298.0 & 2 & HQOXAL \\
\hline C ${ }_{723} \mathrm{H}$ & $\begin{array}{l}6 \text { N } 2 \text { o } 1 \\
1-(2 H)-\text { phthalazinone }\end{array}$ & 124.0 & 119.01 & 126.0 & 391.0 & 2 & \\
\hline C $8{ }_{724}^{H}$ & $\begin{array}{l}6,2 \text { o } 1 \\
2,3 \text {-dihydroxyquinoxal ine }\end{array}$ & $\mathrm{e}^{107.0}$ & 0.0 & 0.0 & 298.0 & 1 & \\
\hline${ }_{725}^{8} \mathrm{H}$ & $\begin{array}{l}6 \text { N } 2 \text { o } 2 \\
\text { quinoxaline-1,4-dioxide }\end{array}$ & 156.0 & 0.0 & 0.0 & 298.0 & 0 & \\
\hline C ${ }_{726}^{H}$ & $\begin{array}{l}6 \text { N } 2 \text { o } 2 \\
3 \text {-aminophthalimide }\end{array}$ & 112.0 & 0.0 & 0.0 & 0.0 & 0 & \\
\hline C ${ }_{727} \mathrm{H}$ & $\begin{array}{l}6 \text { N } 2 \text { o } 2 \\
\text { 4-aminophthalimide }\end{array}$ & 108.0 & 0.0 & 0.0 & 401.0 & 1 & MODYAG \\
\hline C ${ }_{728} \mathrm{H}$ & $\begin{array}{l}6 \text { N } 2 \text { o } 2 \\
5 \text {-nitroindole }\end{array}$ & 135.0 & 0.0 & 0.0 & 459.0 & 1 & \\
\hline C ${ }_{729}{ }^{H}$ & $\begin{array}{l}6 \mathrm{~N} 202 \\
\text { monobenzo-1,3,4,6 -tetr }\end{array}$ & $\begin{array}{l}110.0 \\
\text { raazaper }\end{array}$ & $\begin{array}{c}0.0 \\
\text { entaiene }\end{array}$ & 0.0 & 298.0 & 1 & \\
\hline C $8{ }_{730}^{H}$ & $\begin{array}{l}6 \mathrm{~N} 4 \\
\text { monobenzo-1,3, } 6,6 \text {-tetr }\end{array}$ & $\begin{array}{l}74.9 \\
\text { raazaper }\end{array}$ & $\begin{array}{c}0.0 \\
\text { entalene }\end{array}$ & 0.0 & 348.0 & 1 & \\
\hline C ${ }_{731} \mathrm{H}$ & $\begin{array}{l}6 \mathrm{~N} 4 \\
\text { piperonal }\end{array}$ & 63.6 & 00 & 0.0 & 353.0 & 1 & \\
\hline C ${ }_{732} \mathrm{H}$ & $\begin{array}{l}603 \\
\text { phthalic acid }\end{array}$ & 90.8 & 0.0 & 0.0 & 323.0 & 1 & PHTHAC \\
\hline C ${ }_{733}{ }^{H}$ & $\begin{array}{l}604 \\
\text { isophthalic acid }\end{array}$ & 130.0 & 0.0 & 0.0 & 298.0 & 0 & BFN7DC \\
\hline C ${ }_{734}{ }^{H}$ & $\begin{array}{l}604 \\
\text { terephthalic acid }\end{array}$ & 142.0 & 0.0 & 0.0 & 298.0 & 2 & BEINZUC \\
\hline C ${ }_{735} \mathrm{H}$ & $\begin{array}{l}604 \\
1,3 \text {-benzodioxole-5-carbo }\end{array}$ & $\begin{aligned} 146.0 \\
\text { oxy } 1 \text { ic }\end{aligned}$ & 0.0 & 0.0 & 298.0 & 2 & \\
\hline C ${ }_{736} \mathrm{H}$ & $\begin{array}{l}6 \text { o } 4 \\
\text { benzo (b) thiophene }\end{array}$ & 117.0 & 0.0 & 0.0 & 298.0 & 1 & \\
\hline${ }_{737}{ }^{\mathrm{H}}$ & 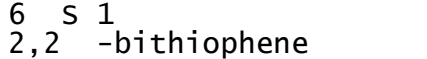 & 66.0 & 0.0 & 0.0 & 298.0 & 0 & DTENYL \\
\hline C ${ }_{738}{ }^{\mathrm{H}}$ & $3,3^{5}$-bithiophene & 85.6 & 0.0 & 0.0 & 298.0 & 1 & \\
\hline C $8{ }_{739} \mathrm{H}$ & $\begin{array}{l}6 \text { S } 2 \\
2-c h 1 \text { oroacetophenone }\end{array}$ & 88.9 & 0.0 & 0.0 & 298.0 & $\perp$ & \\
\hline${ }_{740}^{8} \mathrm{H}$ & 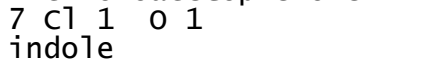 & 90.7 & 0.0 & 0.0 & 293.0 & 1 & INDOLE \\
\hline C ${ }_{741} \mathrm{H}$ & $\begin{array}{l}7 \mathrm{~N} 1 \\
2 \mathrm{H}-1,4 \text {-benzoxazin-3 }\end{array}$ & $\begin{array}{l}76.0 \\
\text {-one }\end{array}$ & 70.0 & 78.0 & 298.0 & 5 & \\
\hline${ }_{742} 2^{H}$ & $\begin{array}{l}7 \mathrm{~N} 1 \\
3-\text { nitroacetophenone }\end{array}$ & & 0.0 & 0.0 & 298.0 & 0 & \\
\hline${ }_{743}^{8} \mathrm{H}$ & $\begin{array}{l}7, \mathrm{~N} 1 \text { o } 3 \\
2,3-\text { dihydro-6-nitro- } 1\end{array}$ & $\begin{array}{c}110.0 \\
\text {-benzod }\end{array}$ & $\begin{array}{r}0.0 \\
\text { dioxin }\end{array}$ & 0.0 & 208.0 & 0 & \\
\hline C ${ }_{744}^{8}{ }^{H}$ & $\begin{array}{l}7 \mathrm{~N} 1 \text { o } 4 \\
\text { 2-methy } 1-3 \text {-nitrobenzoic }\end{array}$ & $\begin{array}{l}101.0 \\
\text { acid }\end{array}$ & 0.0 & 0.0 & 298.0 & 0 & \\
\hline
\end{tabular}




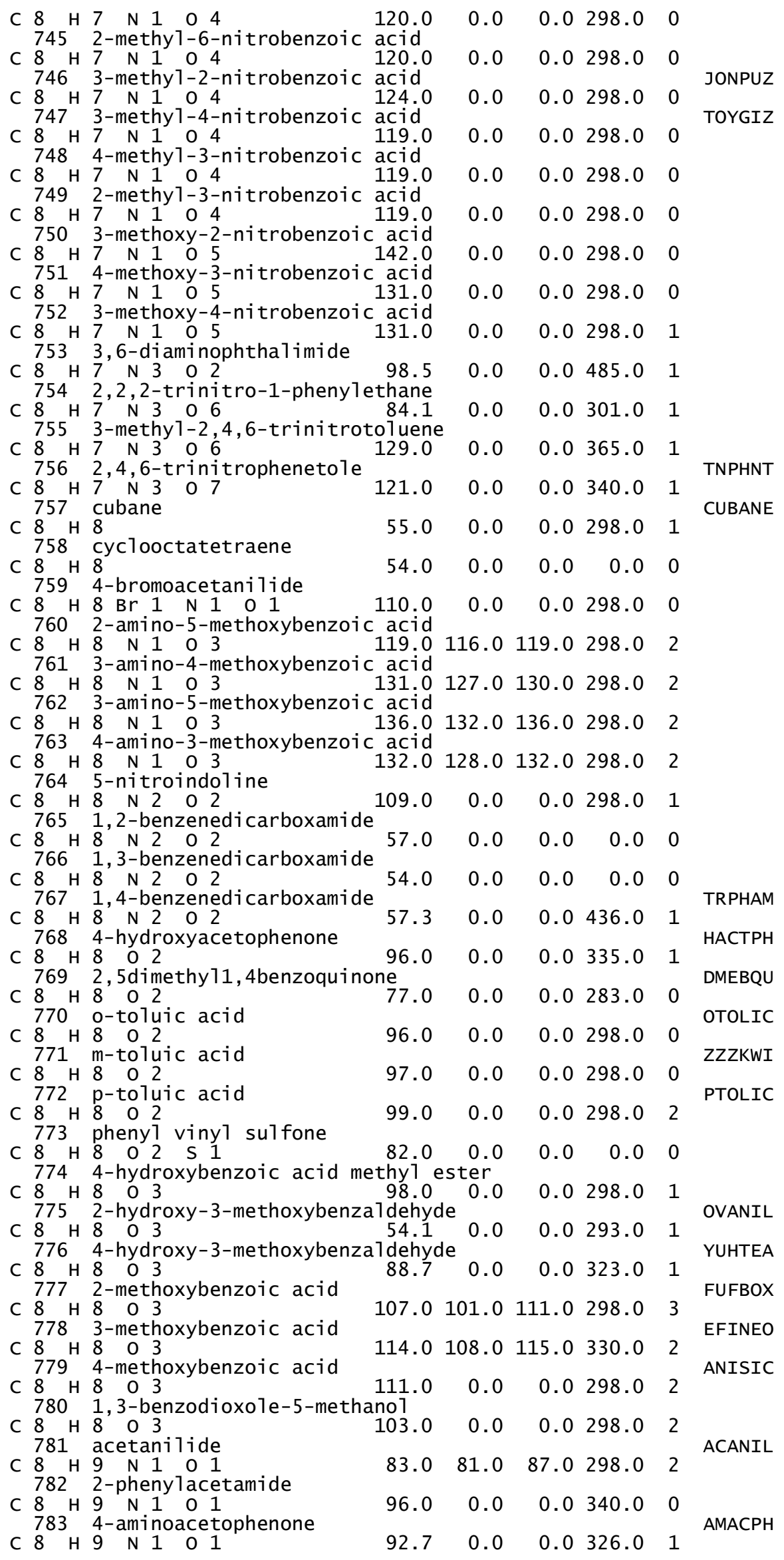




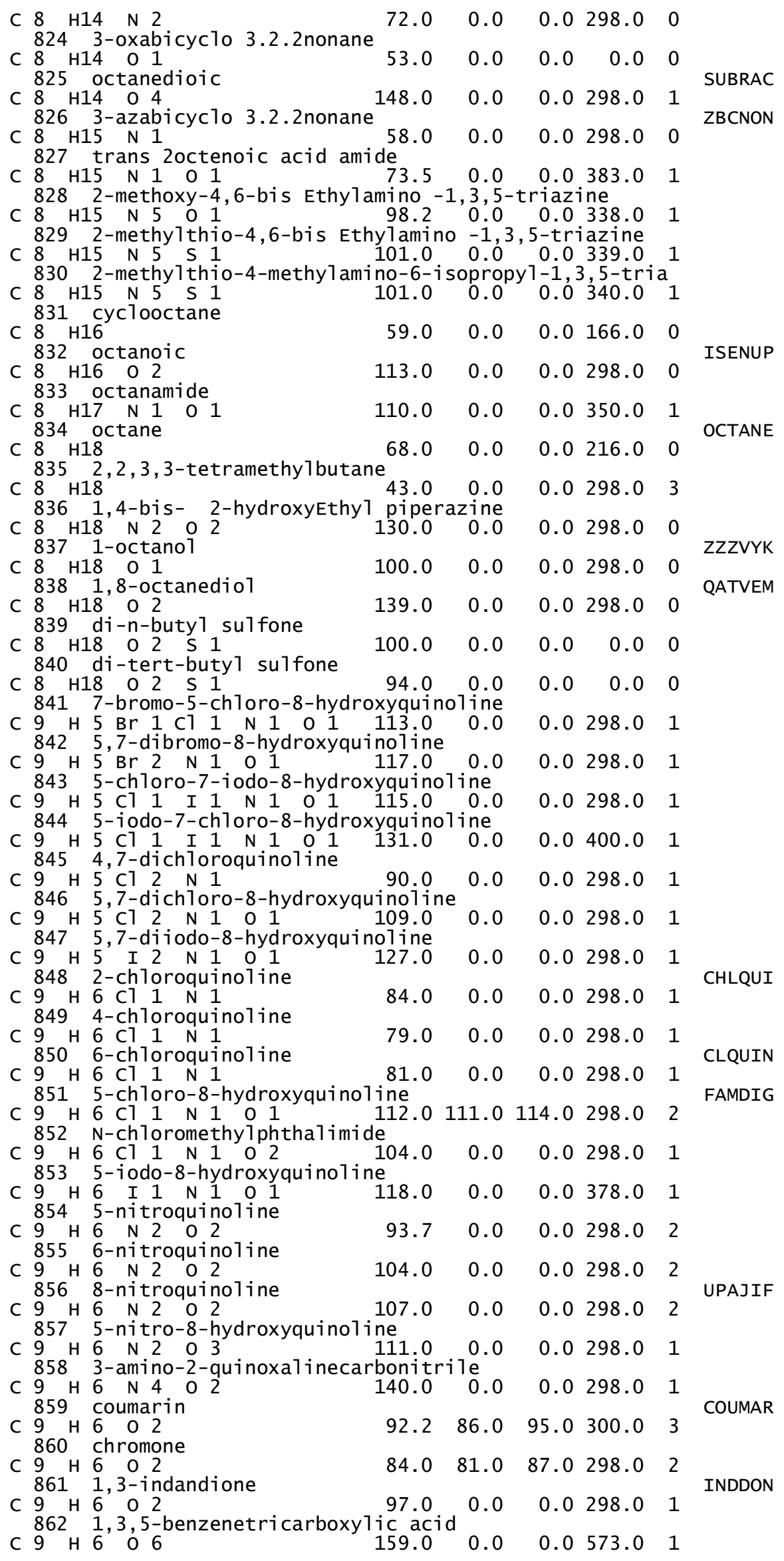




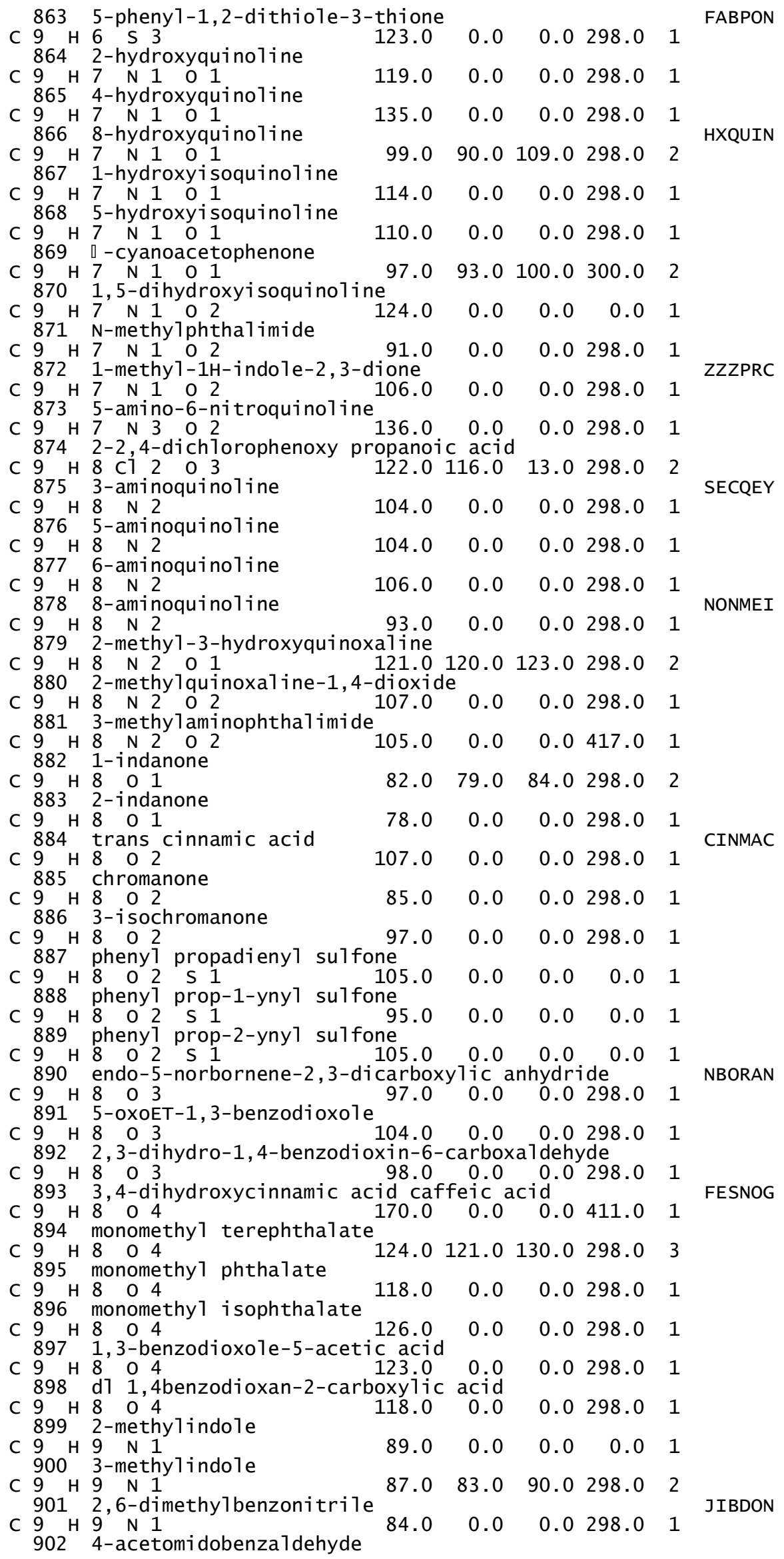




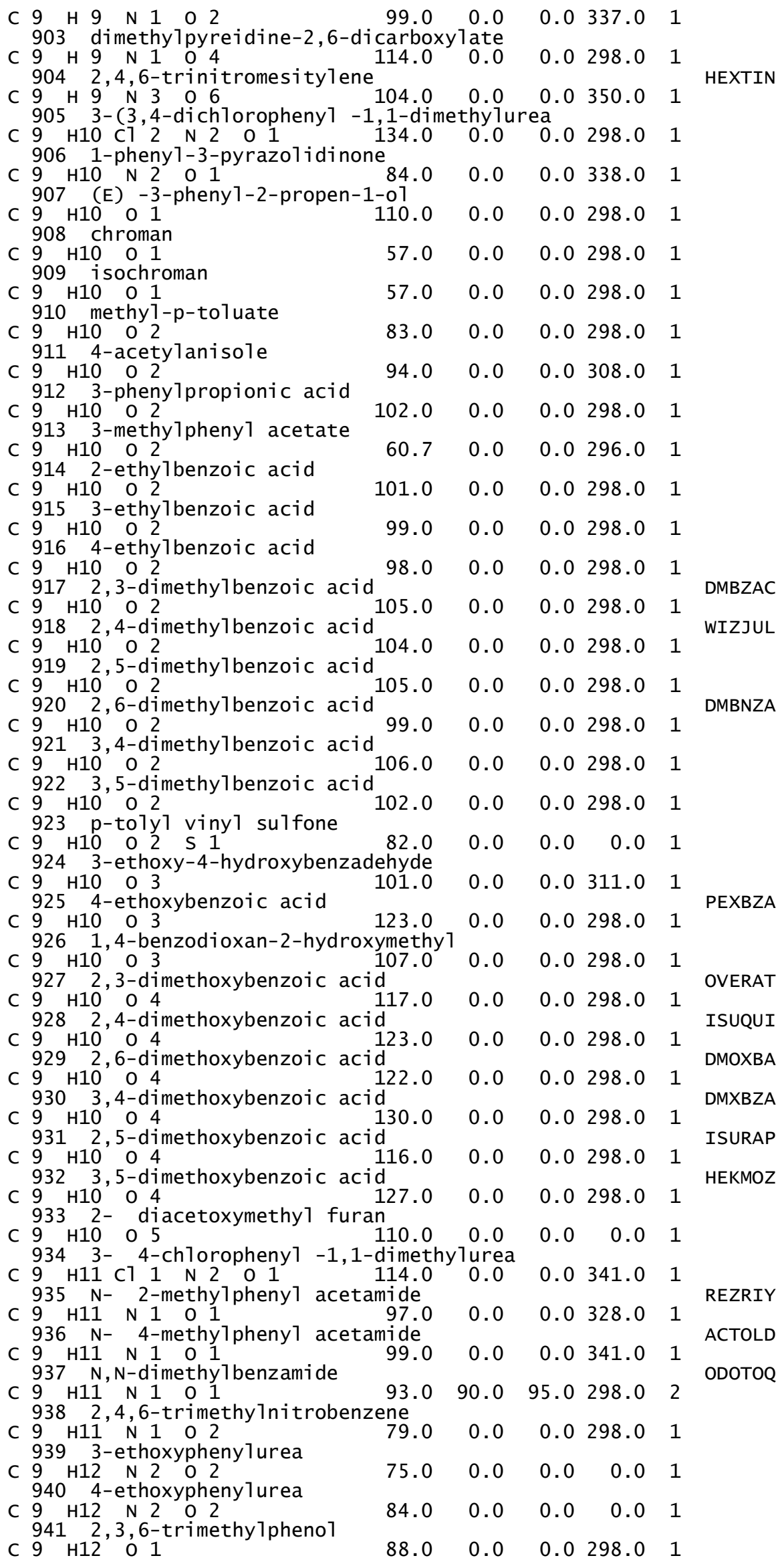




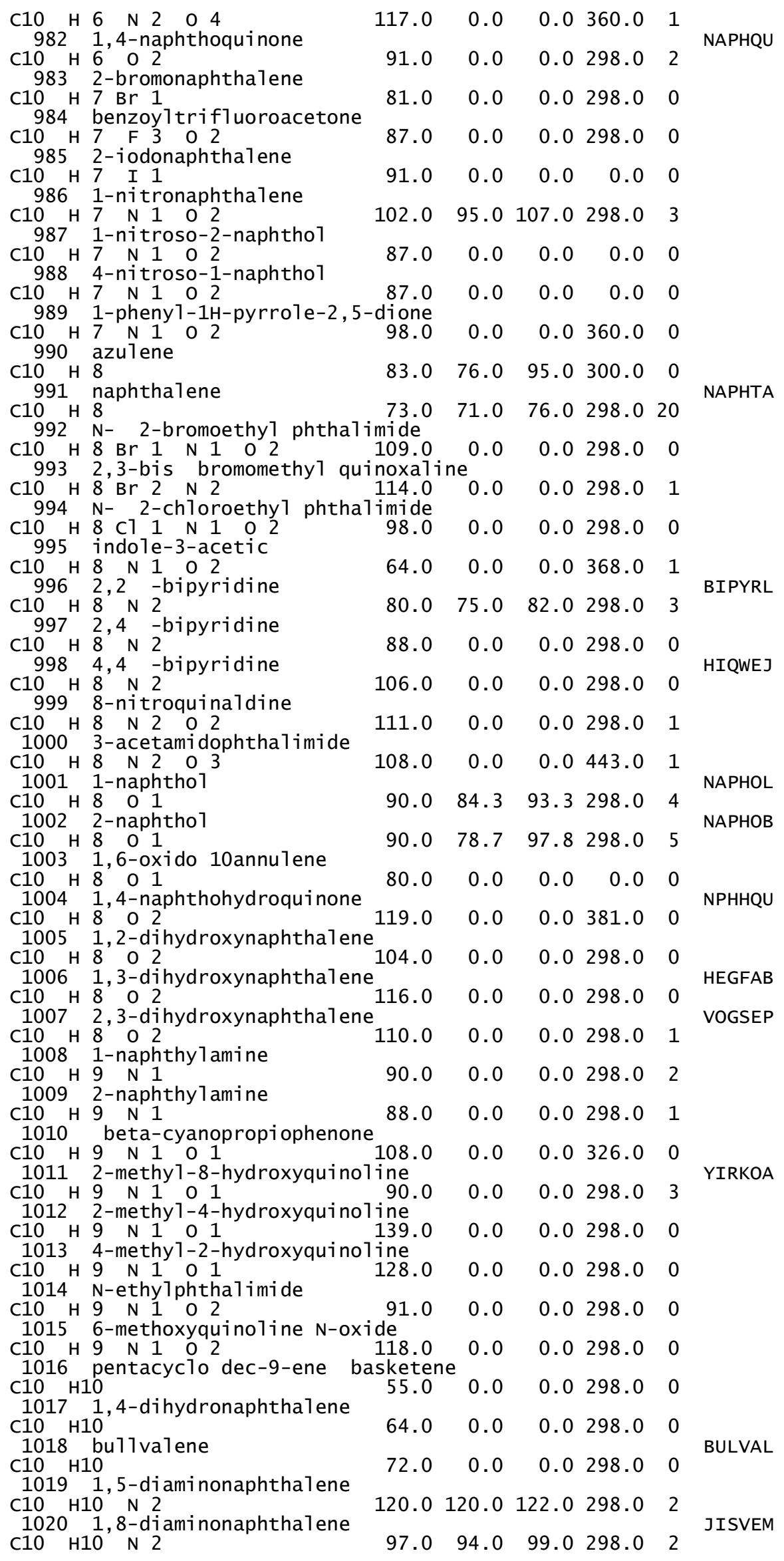




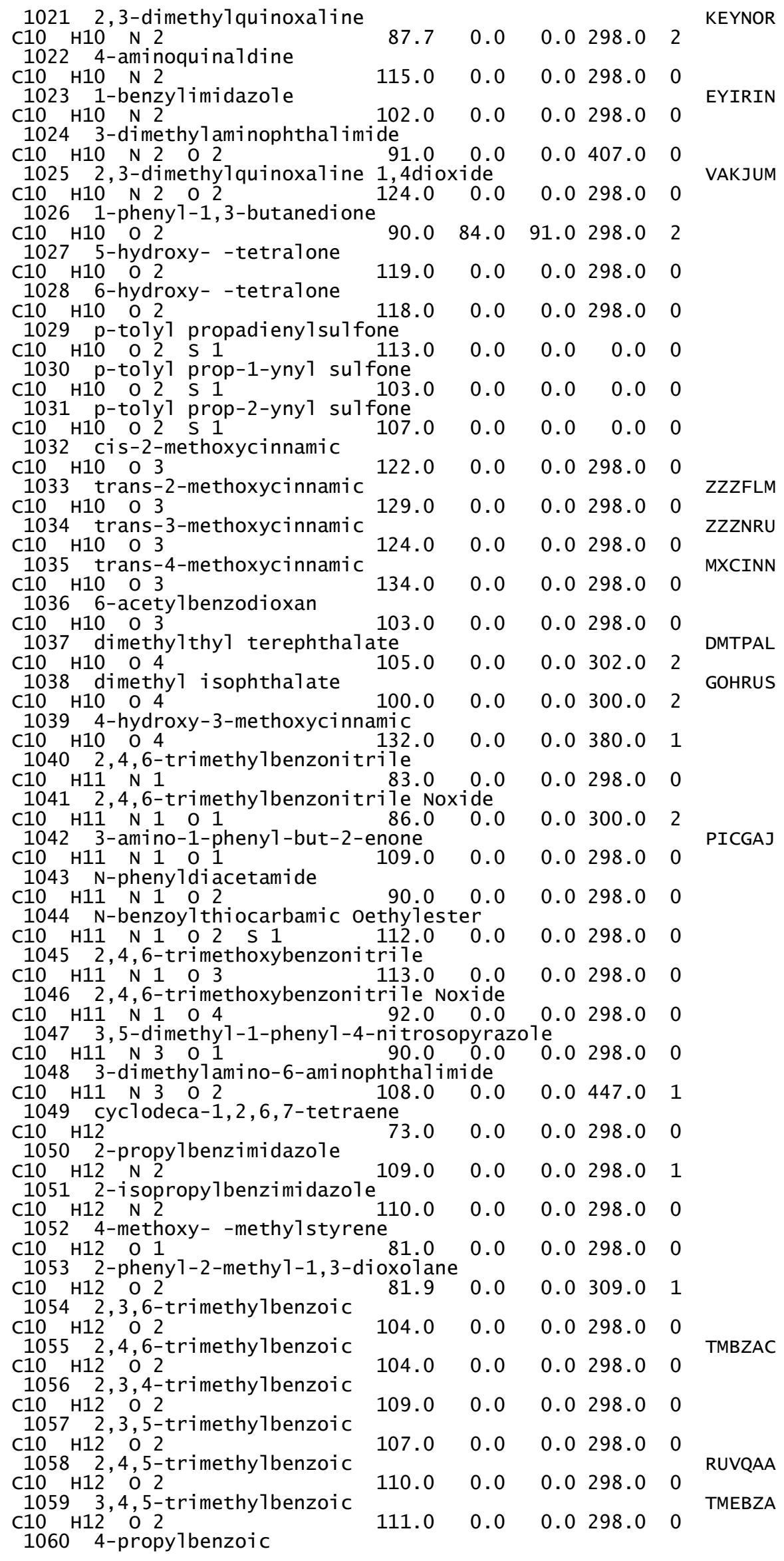




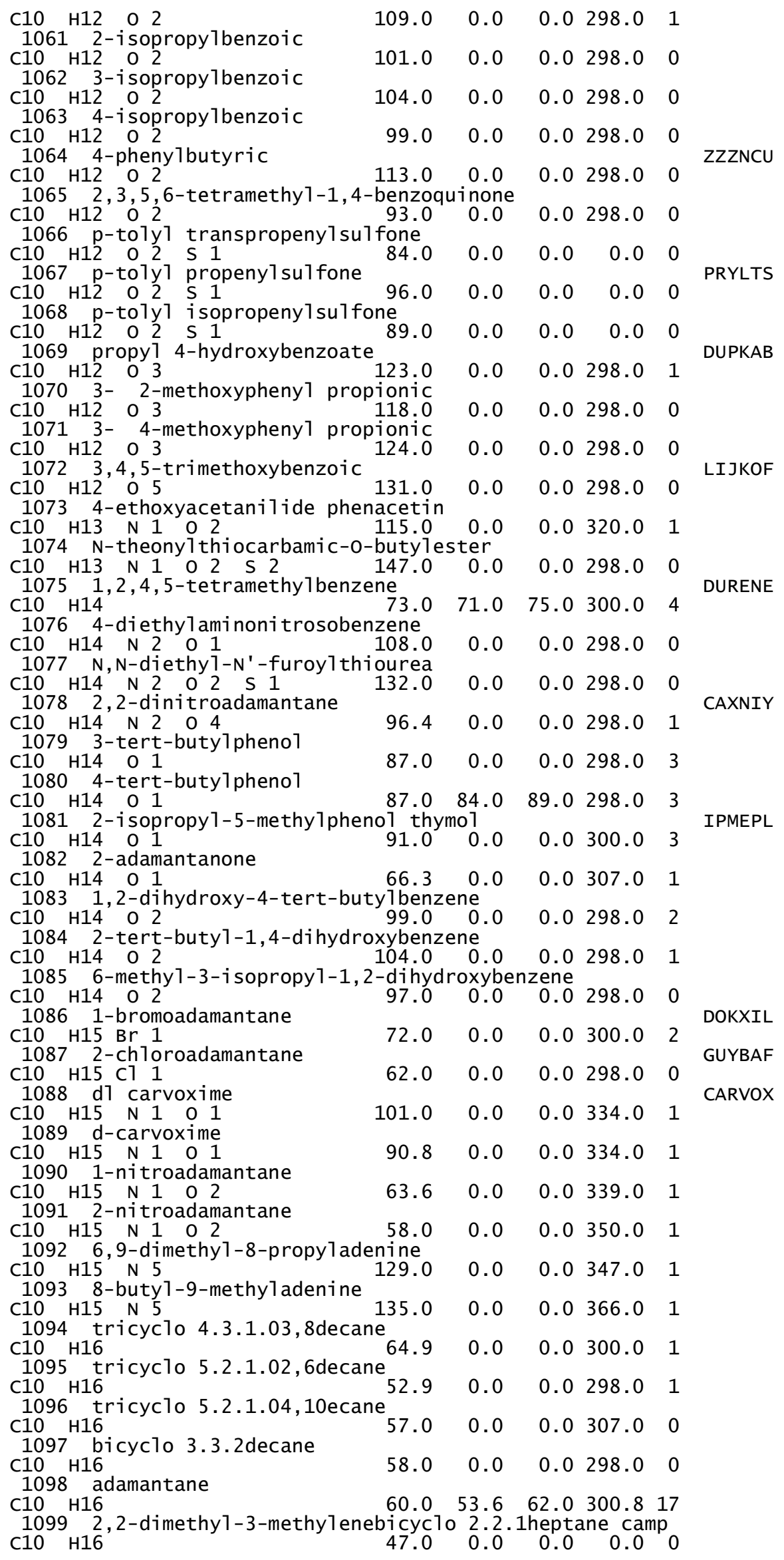




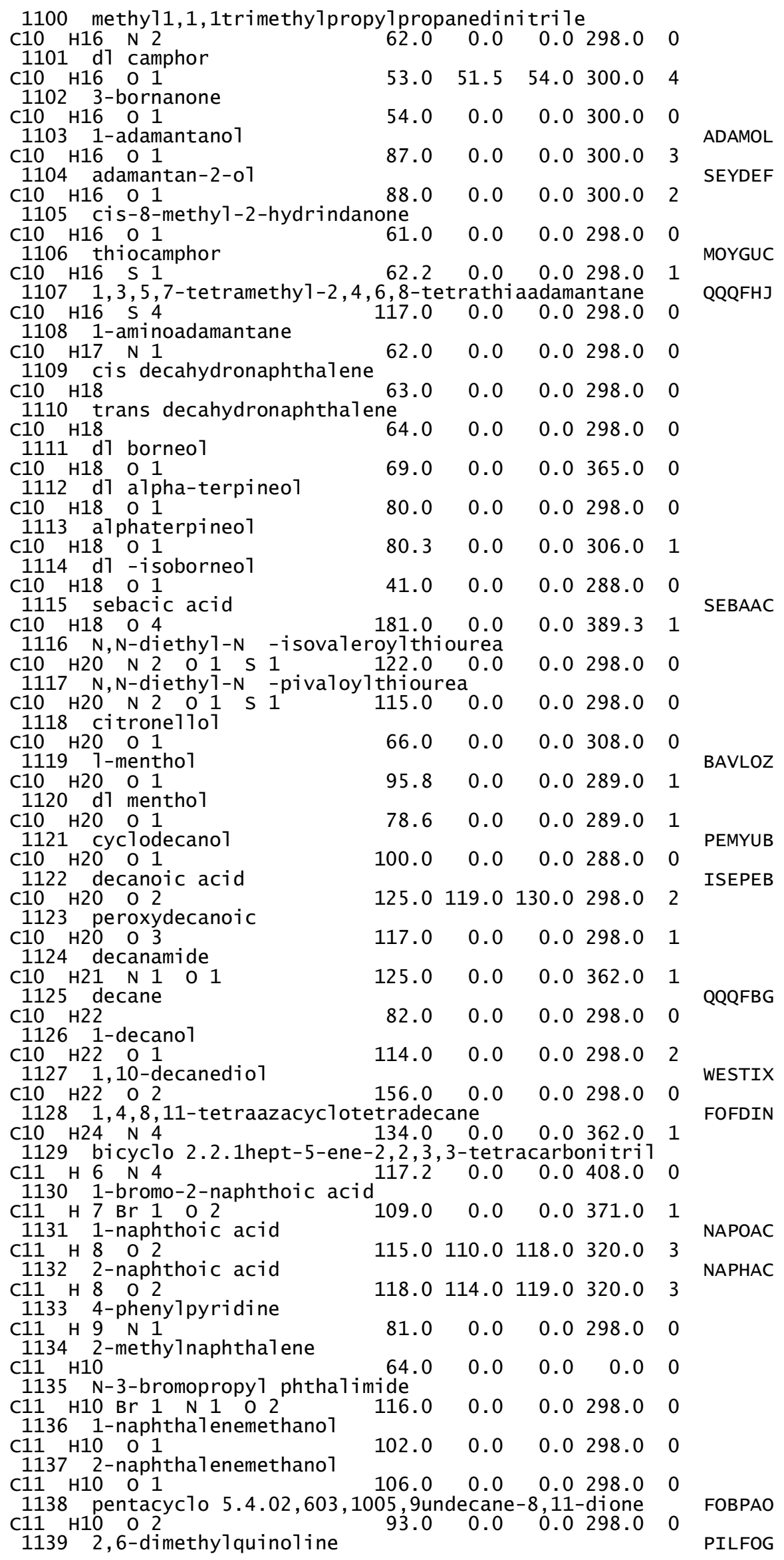




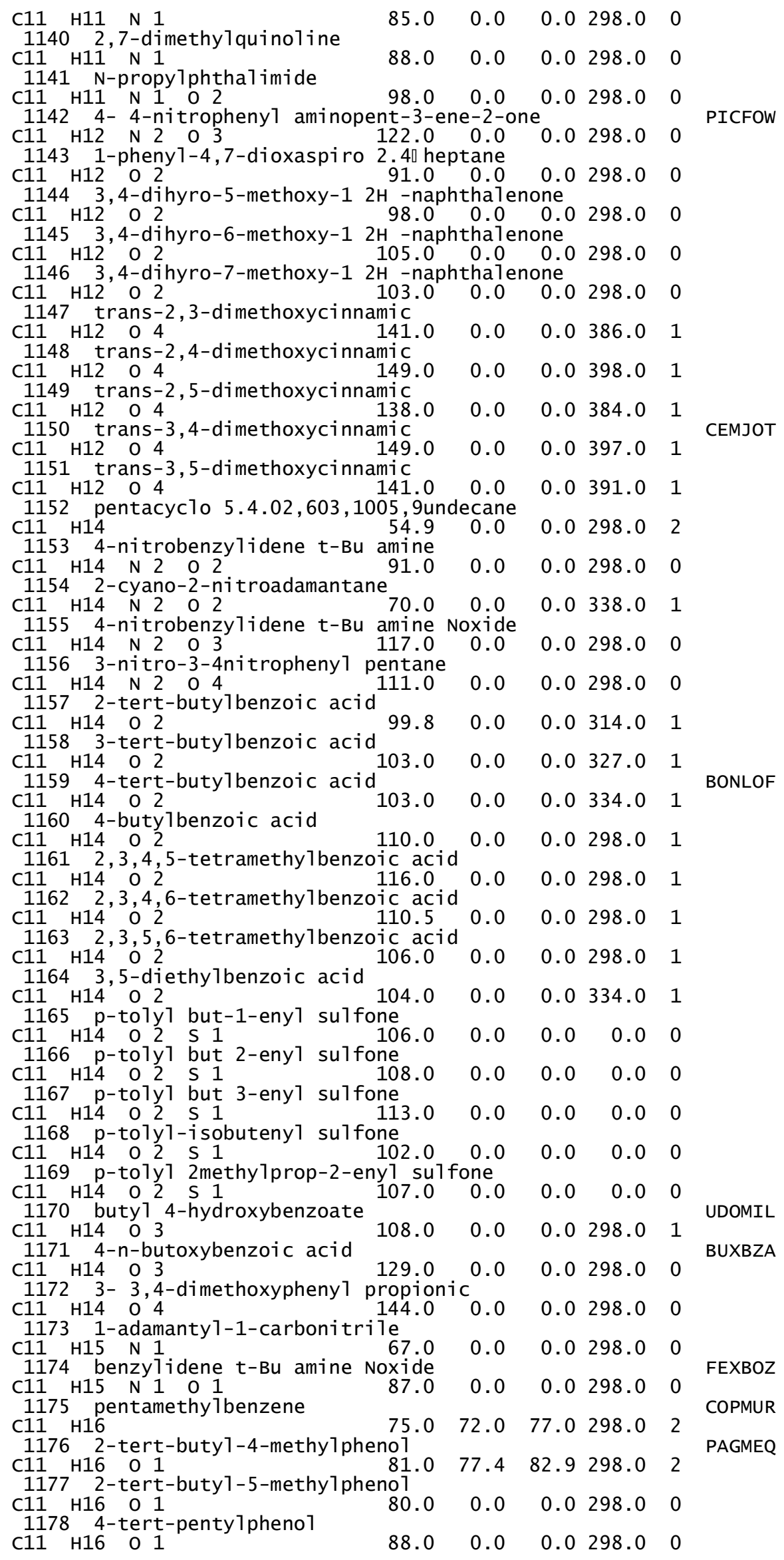




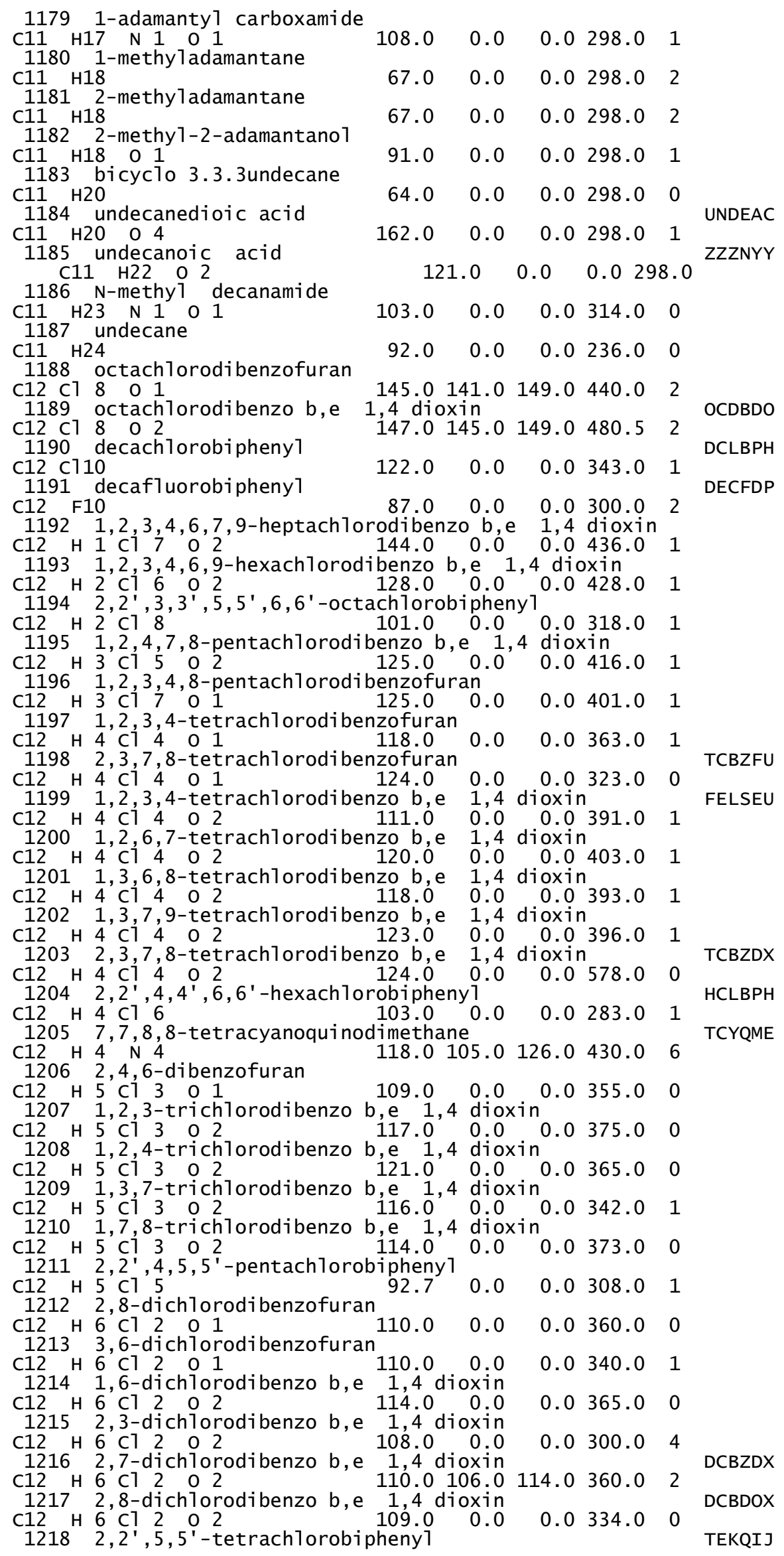




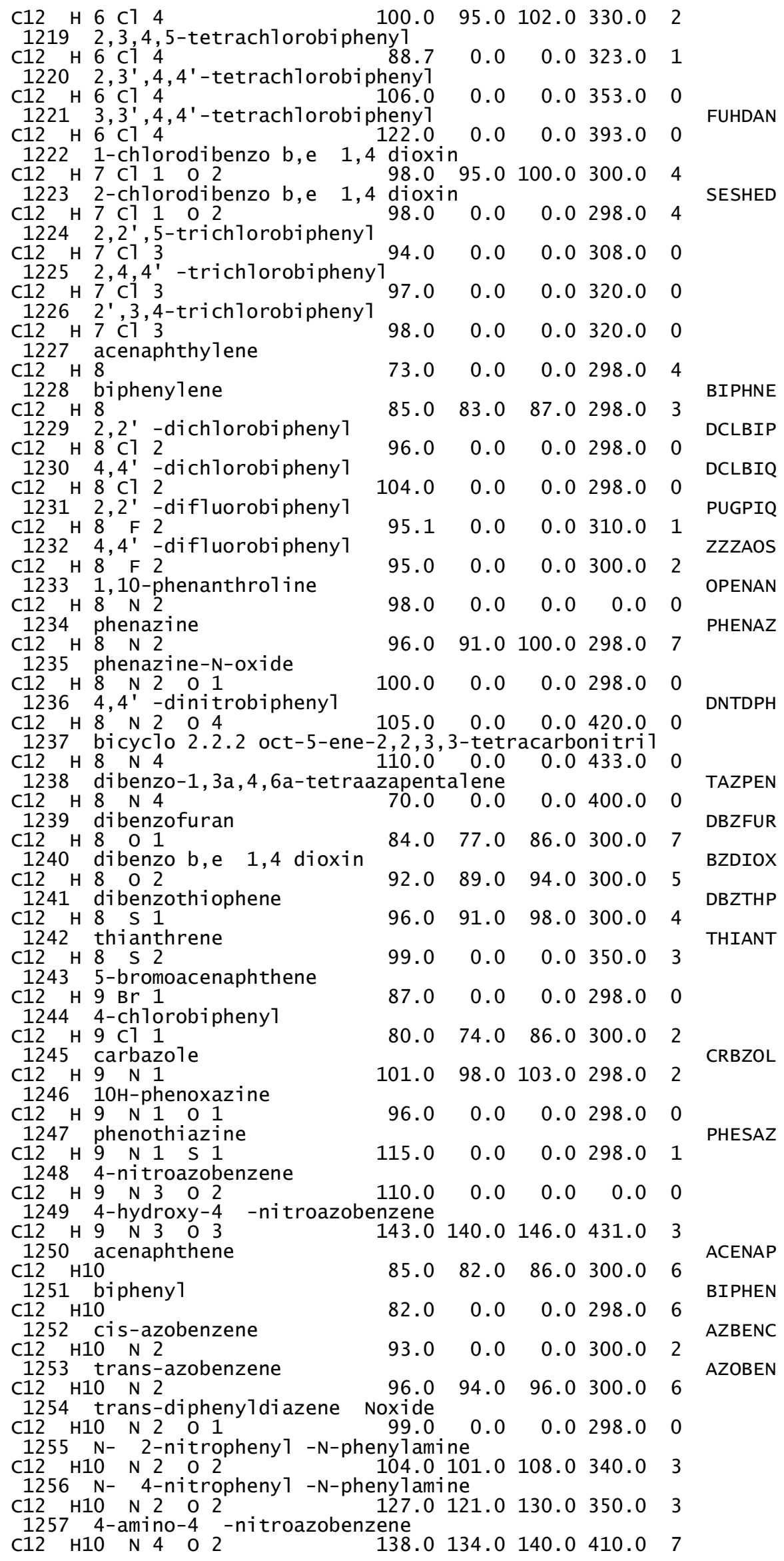




\begin{tabular}{|c|c|c|c|c|c|c|}
\hline $\begin{array}{l}1258 \text { 2-acetylnaphthalene } \\
\mathrm{C} 12 \mathrm{H} 10 \mathrm{O} 1\end{array}$ & 88.0 & 0.0 & 0.0 & 305.0 & 0 & \\
\hline $\begin{array}{l}1259 \text { dipheny } 1 \text { ether } \\
\text { C12 H10 o } 1 \text {. }\end{array}$ & 82.0 & 0.0 & 0.0 & 0.0 & 0 & RAFFIO \\
\hline $\begin{array}{l}1260 \text { 2-hydroxybiphenyl } \\
\text { C12 H10 O } 1 \\
1261 \text { 4-hvdroxybipheny } 1\end{array}$ & 87.0 & 83.0 & 89.0 & 300.0 & 2 & \\
\hline $\begin{array}{l}1261 \text { 4-hydroxybiphenyl } \\
\text { C12 } \mathrm{H} 10 \mathrm{O} 1 \\
1262 \\
2\end{array}$ & 110.0 & 0.0 & 0.0 & 298.0 & 0 & BUPSA \\
\hline $12633^{4,4} 4^{\prime}$-dihydroxybipheny & 114.0 & 0.0 & 0.0 & 298.0 & 0 & NUTSUQ \\
\hline $\begin{array}{c}\mathrm{C} 12 \\
1264{ }^{\mathrm{H}}{ }^{2} \text { alpha } \\
\mathrm{O}\end{array}$ & 143.0 & 0.0 & 0.0 & 298.0 & 0 & PARPEF \\
\hline 1265 beta ${ }_{12}$-naphthy 1 acetate & 95.0 & 0.0 & 0.0 & 298.0 & 1 & The \\
\hline $\mathrm{C} 12 \mathrm{H} 10$ O 2 & 96.3 & 0.0 & 0.0 & 298.0 & 1 & NAPACA \\
\hline $\mathrm{C} 12 \mathrm{H} 10 \mathrm{O} 2$ & 112.0 & 0.0 & 0.0 & 298.0 & 1 & NAPA \\
\hline $\mathrm{C} 12 \mathrm{H} 1 \mathrm{H}^{\mathrm{C}}$ & 124.0 & 0.0 & 0.0 & 298.0 & 0 & PEYG \\
\hline $\begin{array}{l}\mathrm{C} 12 \\
1269 \mathrm{H} 10 \mathrm{O} 2 \mathrm{~S} \text { diphenyl disulfone }\end{array}$ & 106.0 & 0.0 & 0.0 & 0.0 & 0 & DPSUI \\
\hline 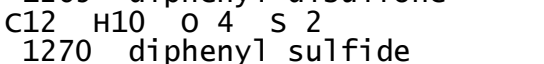 & 162.0 & 0.0 & 0.0 & 0.0 & 0 & ש \\
\hline $\begin{array}{l}\mathrm{C} 12 \mathrm{H} 10 \mathrm{~S} 1 \\
1271 \text { diphenylamine }\end{array}$ & 95.0 & 0.0 & 0.0 & 0.0 & 0 & OOOBVP \\
\hline${ }_{1272} \mathrm{H} 11 \mathrm{~N}$-acety $1-1$-naphthylamine & $e^{105.0}$ & 97.0 & 110.0 & 300.0 & 4 & QQQDVR \\
\hline${ }_{1273}{ }^{\mathrm{H} 11}{ }_{4} \mathrm{~N}$-aminoazobenzene & 94.0 & 0.0 & 0.0 & 349.0 & 1 & \\
\hline $1274{ }^{\mathrm{H} 11} \mathrm{~N} 3$ & 110.0 & 0.0 & 0.0 & 364.0 & 1 & DMNA \\
\hline $1275 \quad 2,3$-dimeth & 81.0 & 78.0 & 83.0 & 310.0 & 3 & BIMIVAT \\
\hline $\begin{array}{cc}\mathrm{C} 12 \\
1276\end{array} \mathrm{H}^{2}$, & 83.0 & 0.0 & 0.0 & 310.0 & 2 & \\
\hline${ }_{12} 127^{\mathrm{H} 12} 2,7$-dimethy 7 naphthalene & 83.0 & 0.0 & 0.0 & 300.0 & 2 & DIMINPI \\
\hline $1278{ }^{\mathrm{H} 12} 4,4^{\prime}$-dimethy $1-2-2$ 'bipyr & $\begin{array}{l}84.0 \\
\text { ridy } 1\end{array}$ & 0.0 & 0.0 & 350.0 & 3 & NAMKAN \\
\hline${ }_{12}^{\mathrm{C} 2} 29^{\mathrm{H} 12} 4,4^{\mathrm{N}} 2$-diaminodiphenyl o) & $\begin{array}{l}100.0 \\
\text { xide }\end{array}$ & 0.0 & 0.0 & 298.0 & 0 & \\
\hline${ }_{12} 120_{1}{ }^{12} \mathrm{~N} 2$-dimethylcubane dicar & $\begin{array}{l}63.0 \\
\text { rboxy7a }\end{array}$ & ate 0.0 & 0.0 & 0.0 & 0 & HEGSAN \\
\hline $\begin{array}{l}\mathrm{C} 12 \mathrm{H} 12 \mathrm{O} 4 \\
\mathrm{C} 1281 \mathrm{H}, 3,5 \text {-benzenetricarboxy }\end{array}$ & $\begin{array}{l}117.0 \\
7 i c \text { aci } \\
118.0\end{array}$ & $\begin{array}{c}0.0 \\
\text { id } \\
0.0\end{array}$ & $\begin{array}{c}0.0 \\
\text { heester } \\
0.0\end{array}$ & $\begin{array}{r}298.0 \\
298.0\end{array}$ & 0 & DUJTIM \\
\hline 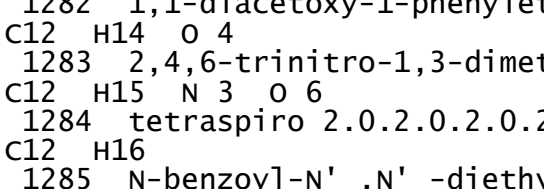 & $\begin{array}{l}\text { thane } \\
94.0 \\
\text { thy } 1-5-5 \\
100.0 \\
2.0 \text { dod } \\
75.0\end{array}$ & $\begin{array}{c}0.0 \\
\text { tert-b } \\
0.0 \\
\text { decane } \\
0.0\end{array}$ & $\begin{array}{l}0.0 \\
\text { utyibe } \\
0.0 \\
4 \text { rotan } \\
0.0\end{array}$ & $\begin{array}{r}318.0 \\
\text { enzene } \\
330.0 \\
\text { ne } \\
318.0\end{array}$ & 1 & $\begin{array}{l}\text { DOHCOS } \\
\text { SCPCBU }\end{array}$ \\
\hline 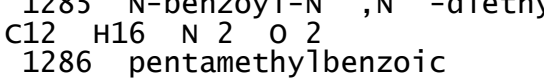 & & 0.0 & 0.0 & 298.0 & 0 & TUS \\
\hline 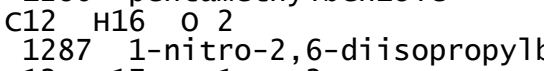 & $\begin{array}{l}113.0 \\
\text { benzene }\end{array}$ & 0.0 & 0.0 & 298.0 & 1 & 105 \\
\hline 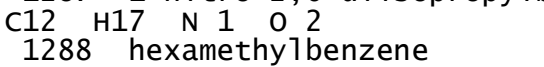 & & 0.0 & 0.0 & 298.0 & 0 & HMBENZ \\
\hline C12 ${ }^{\mathrm{H} 128}{ }^{\mathrm{H}} 1$-adamanty 1 methy 1 chetor & $n e^{84.0}$ & 81.0 & 86.0 & 298.0 & 7 & \\
\hline 1290 1-adamanty $1-1$-carboxy 11 & $\begin{array}{l}84.2 \\
\text { c acid }\end{array}$ & $\begin{array}{l}0.0 \\
\text { Meeste }\end{array}$ & & 298.0 & 1 & KUKLIN \\
\hline 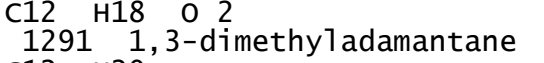 & & & 0.0 & 298.0 & 1 & \\
\hline${ }_{1292}^{\mathrm{C} 12}{ }^{\mathrm{H} 20}$, 2-dimethy 1 adamantane & 68.0 & 0.0 & 0.0 & 298.0 & 0 & \\
\hline${ }_{1293}{ }^{\mathrm{H} 20}$ trans $2 \mathrm{cy}$ & $\begin{array}{r}74.0 \\
\text { xano1 }\end{array}$ & 0.0 & 0.0 & 298.0 & 1 & \\
\hline $\begin{array}{l}\text { C12 H22 O } 1 \\
1294 \quad \text { cyclododecanone }\end{array}$ & & 0.0 & 0.0 & 320.0 & 1 & \\
\hline $\mathrm{C}_{1295}{ }^{\mathrm{H} 22}$ dodecanedioic acid & 83.0 & 0.0 & 0.0 & 298.0 & 0 & DFCDA \\
\hline C12 ${ }_{1296}^{\mathrm{H} 22}$ cyclododecane & 160.0 & 153.0 & 169.0 & 350.0 & 2 & \\
\hline $\mathrm{C} 1297{ }^{\mathrm{H} 24}$ dodecanoic acid & 76.0 & 0.0 & 0.0 & 298.0 & 2 & LAURAC \\
\hline
\end{tabular}




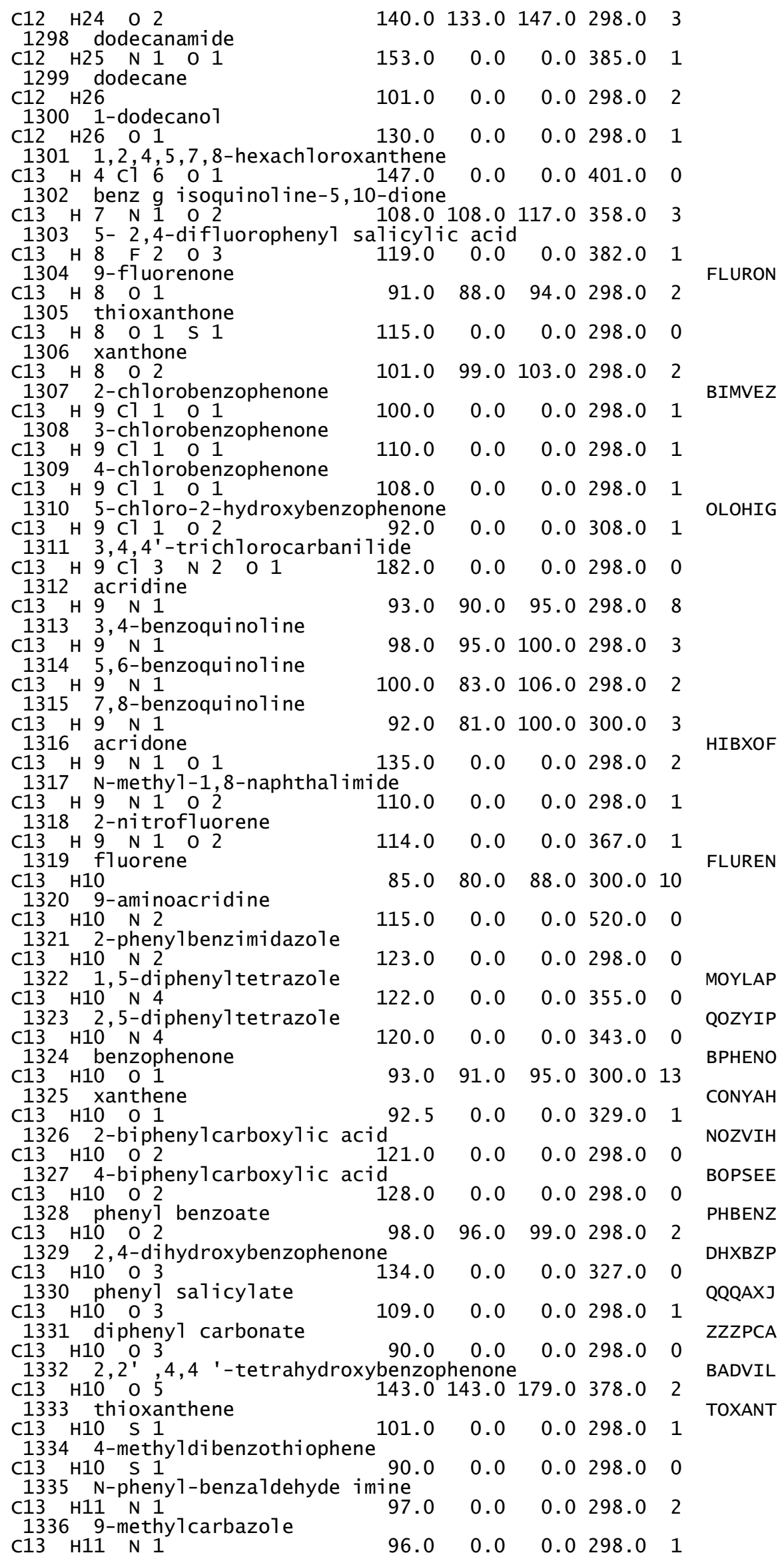




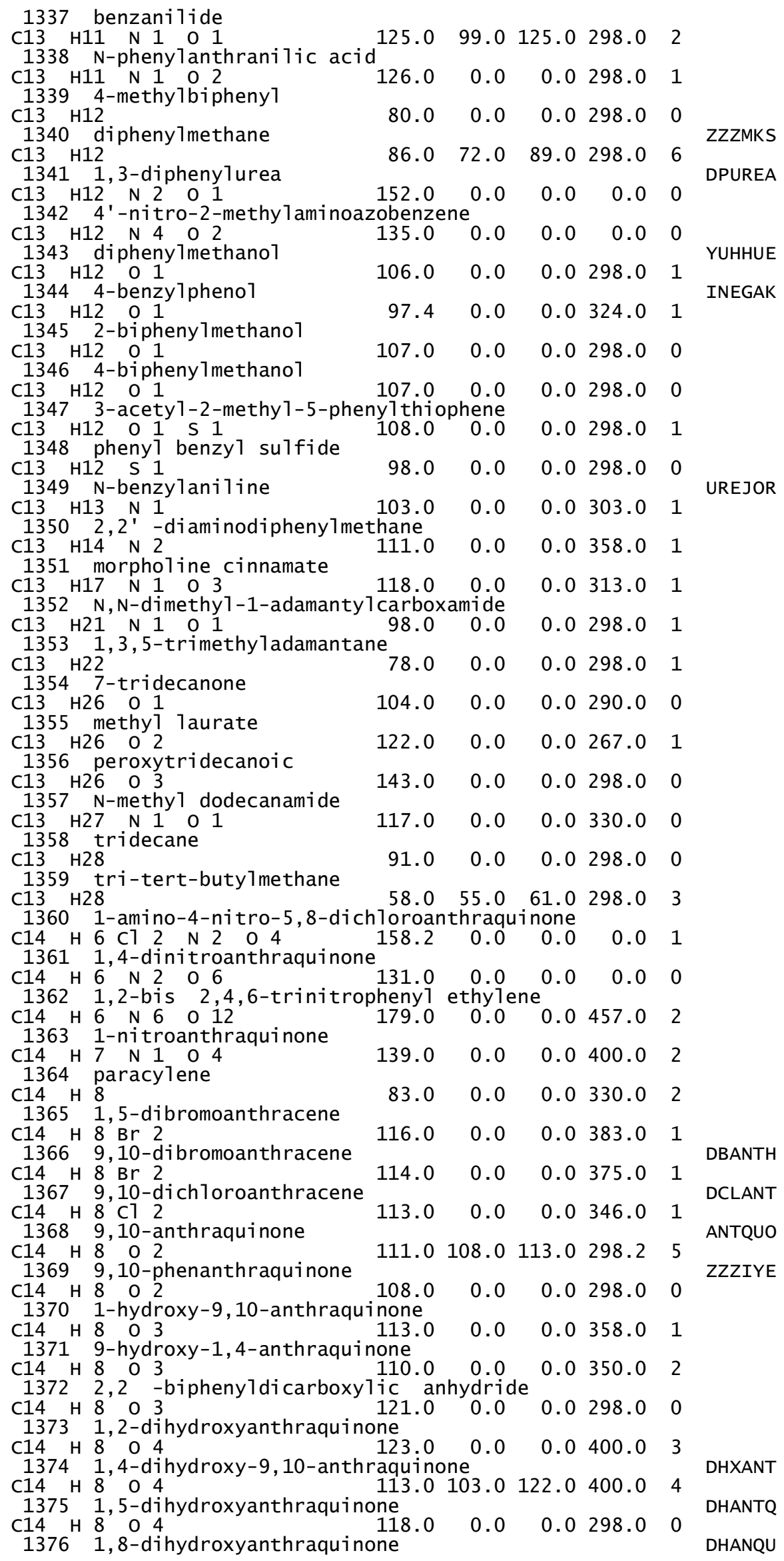




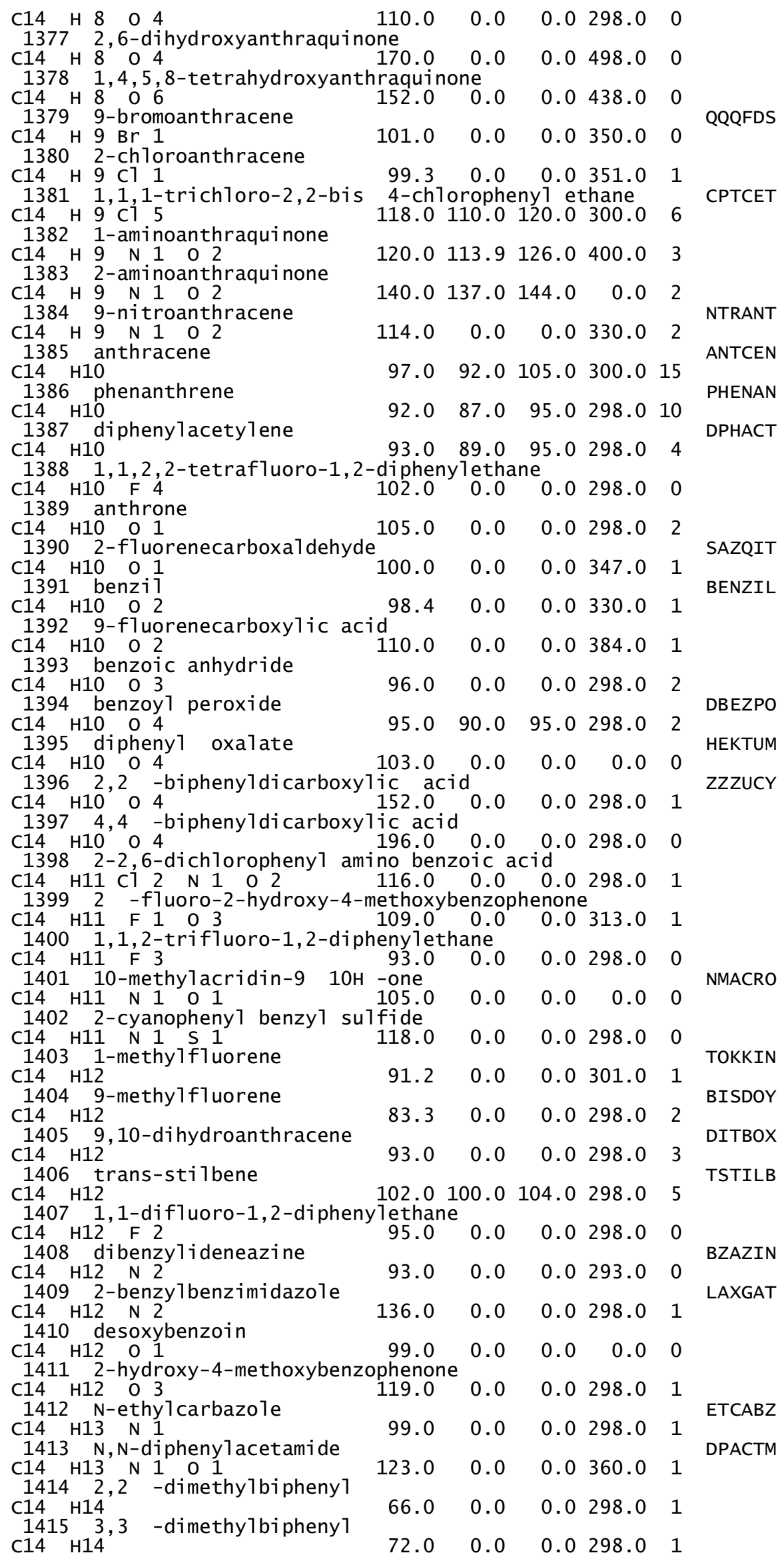




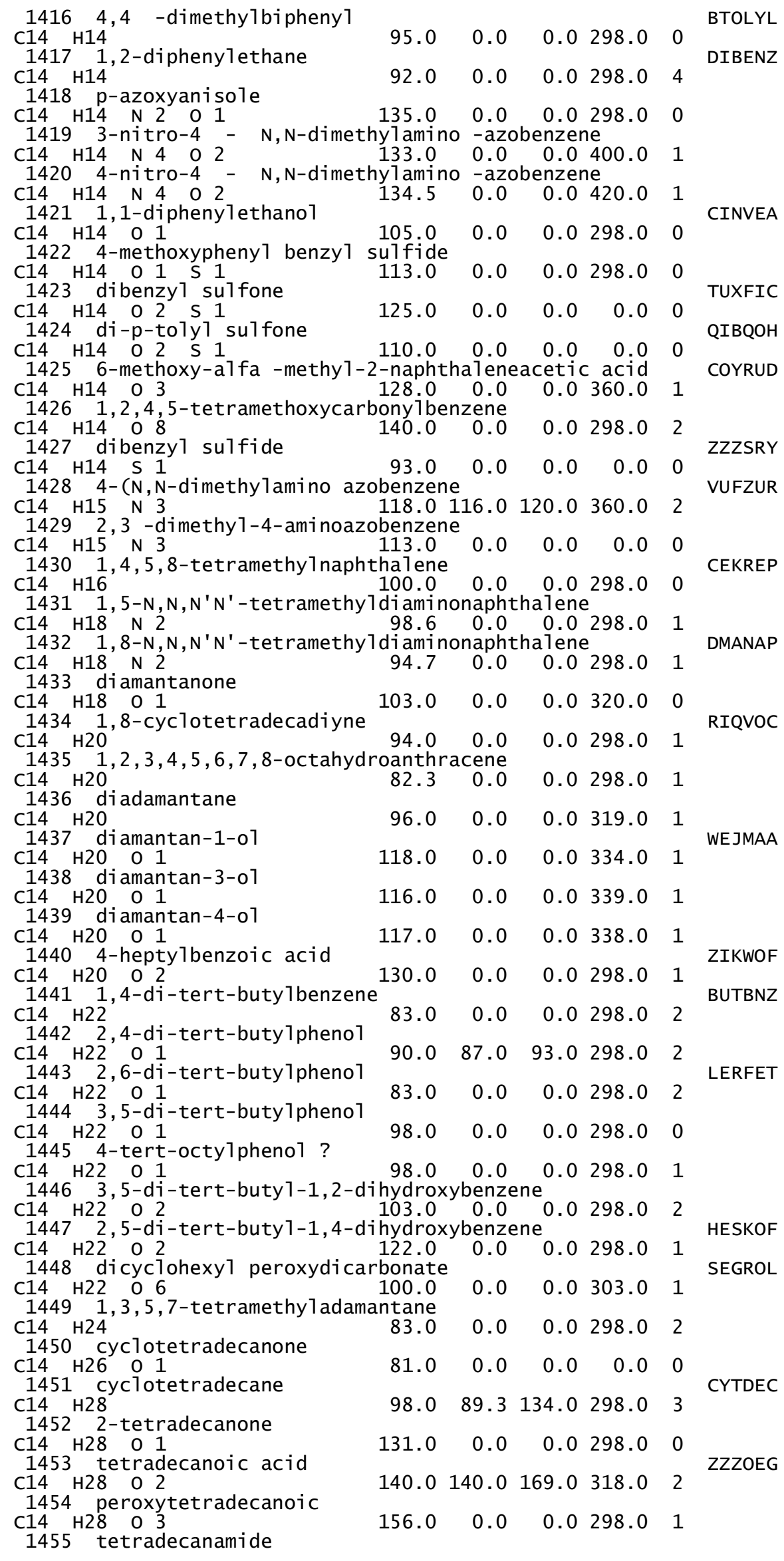




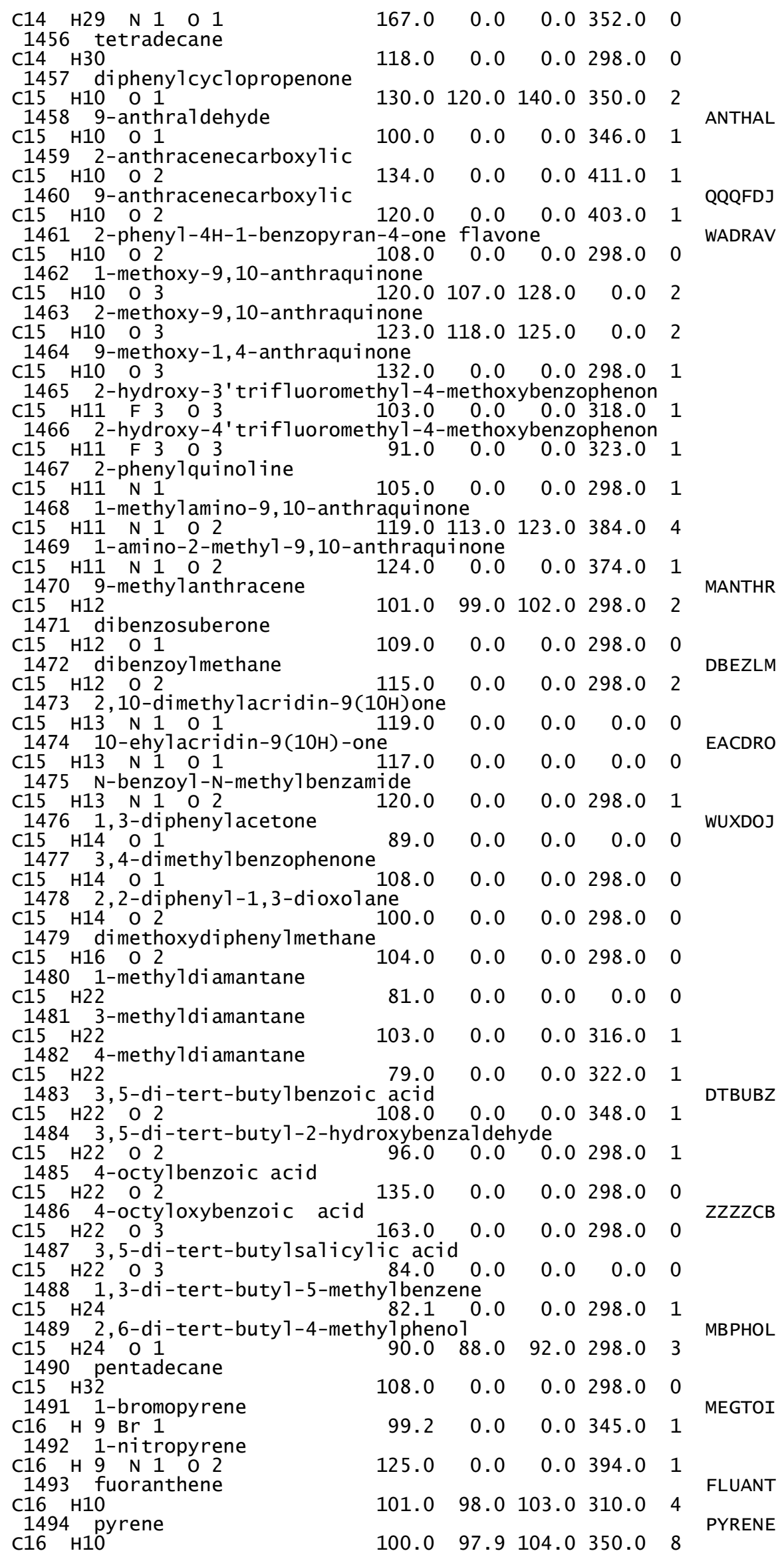




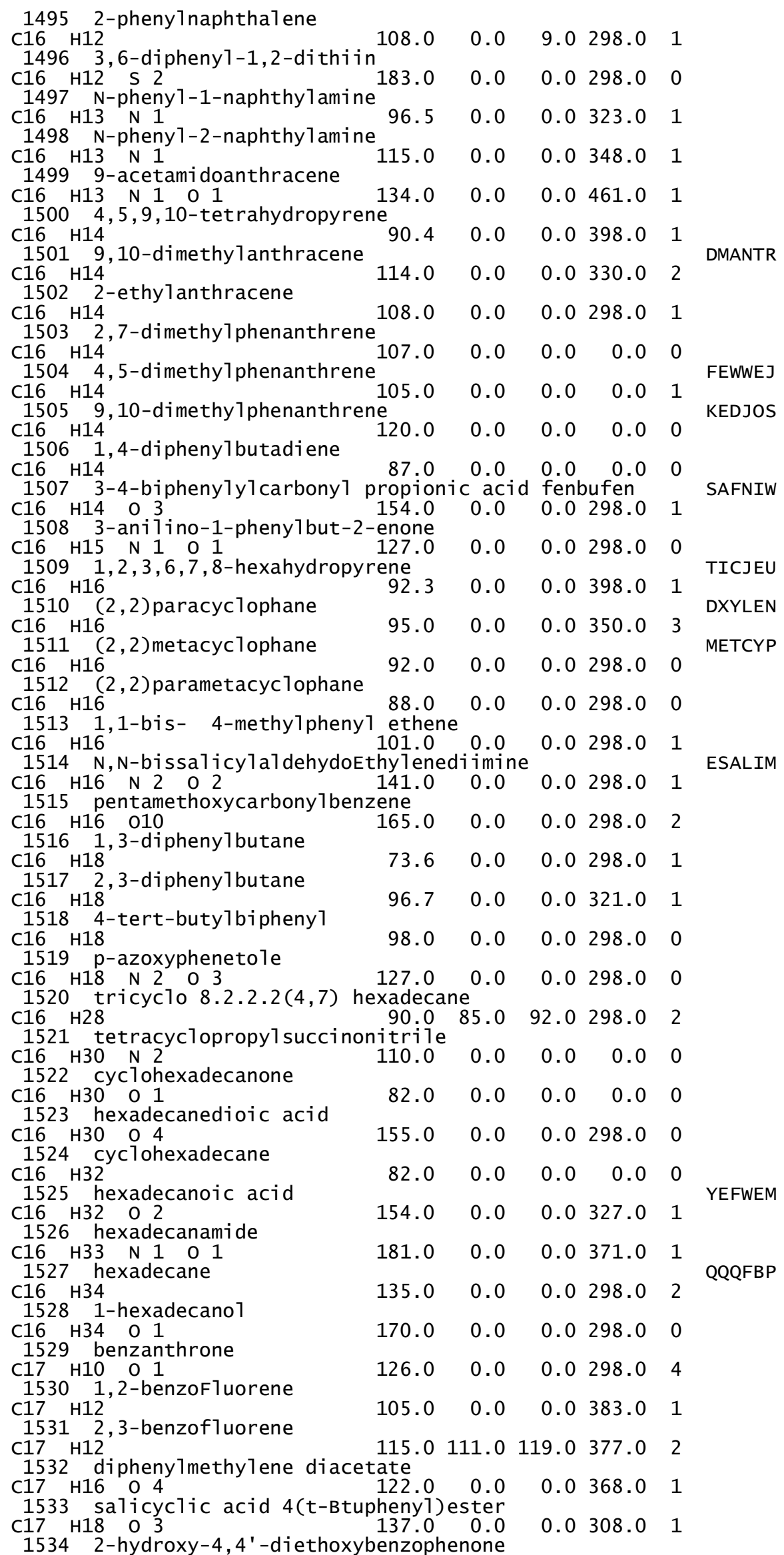




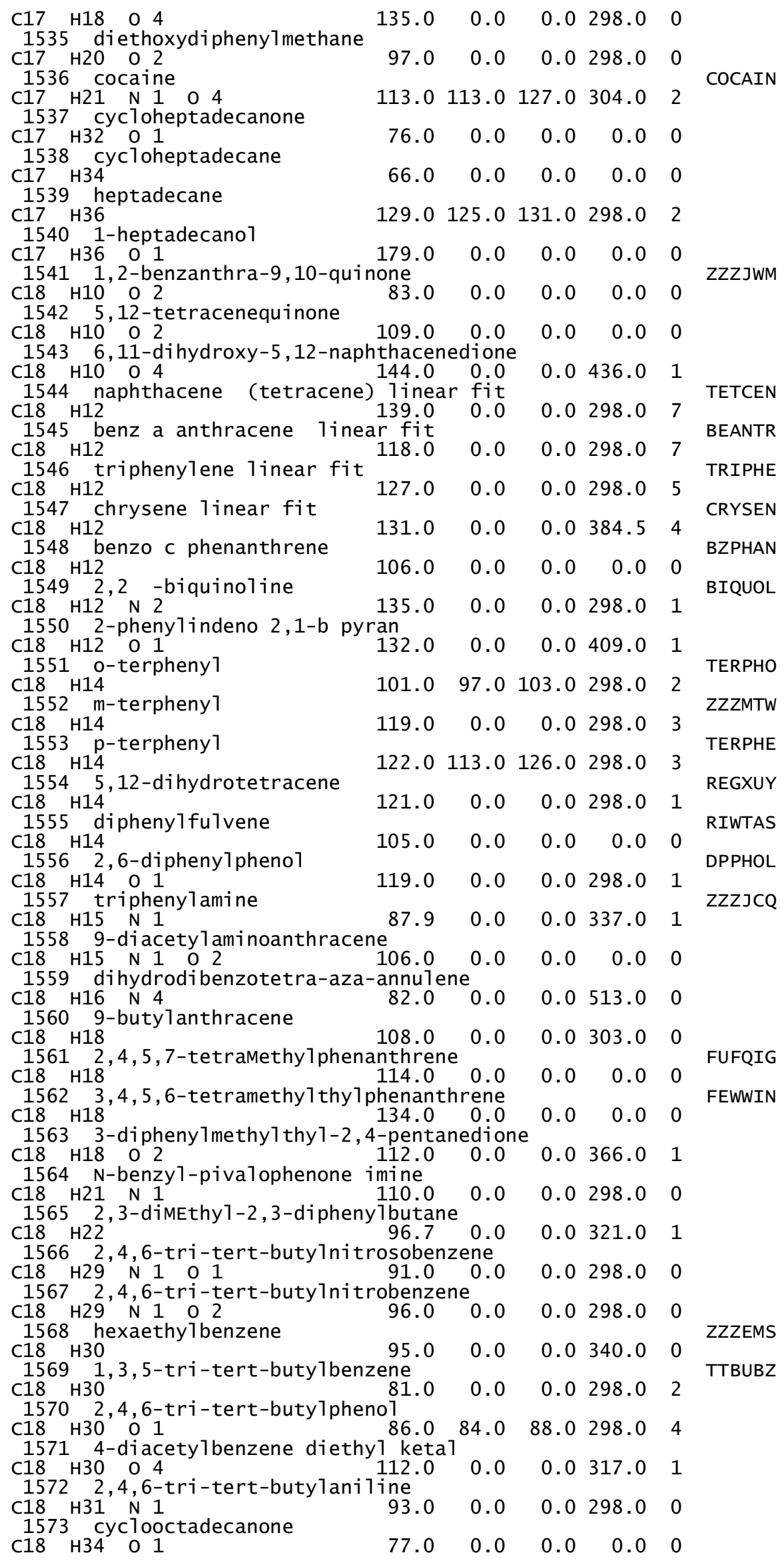




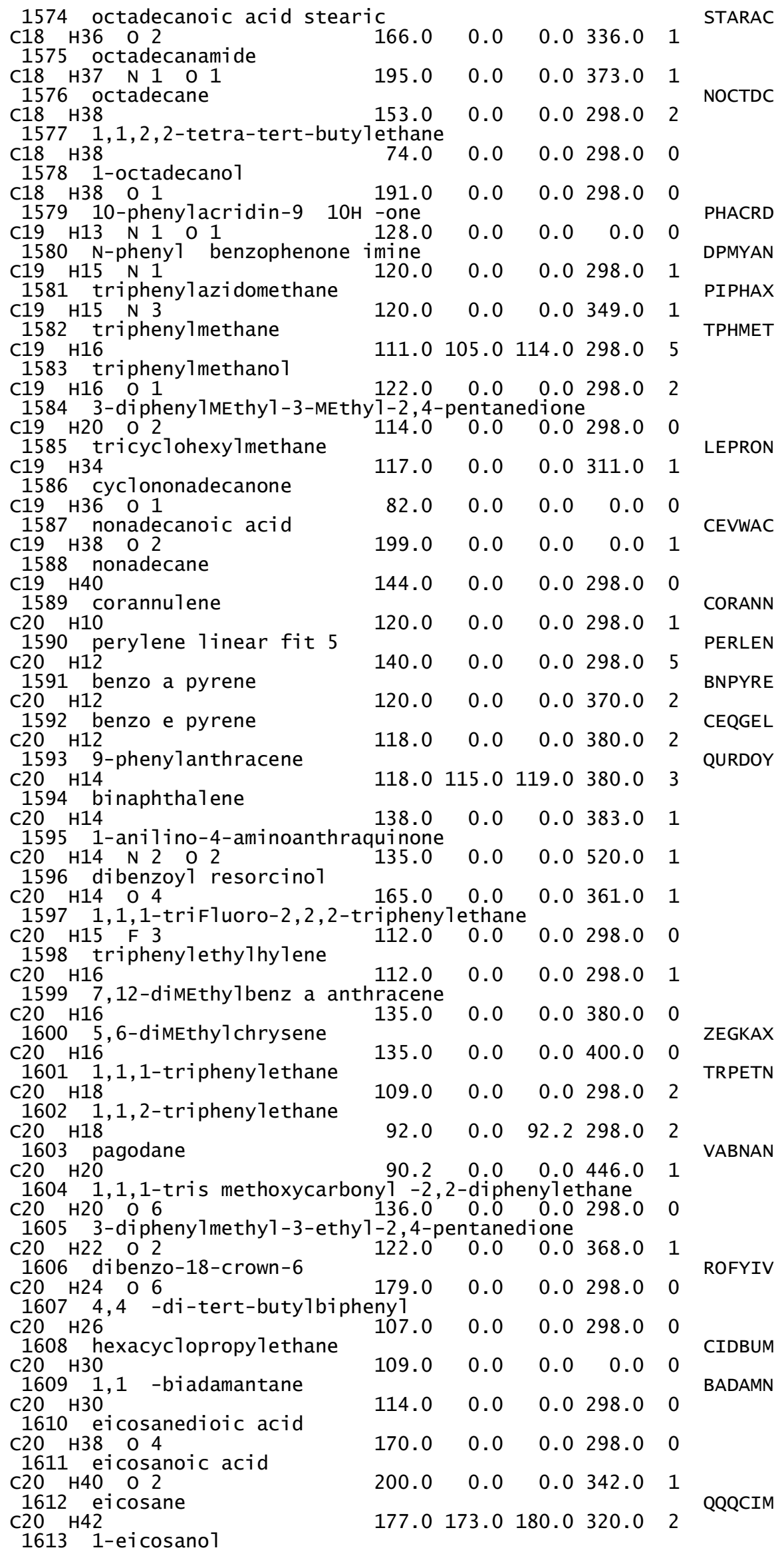




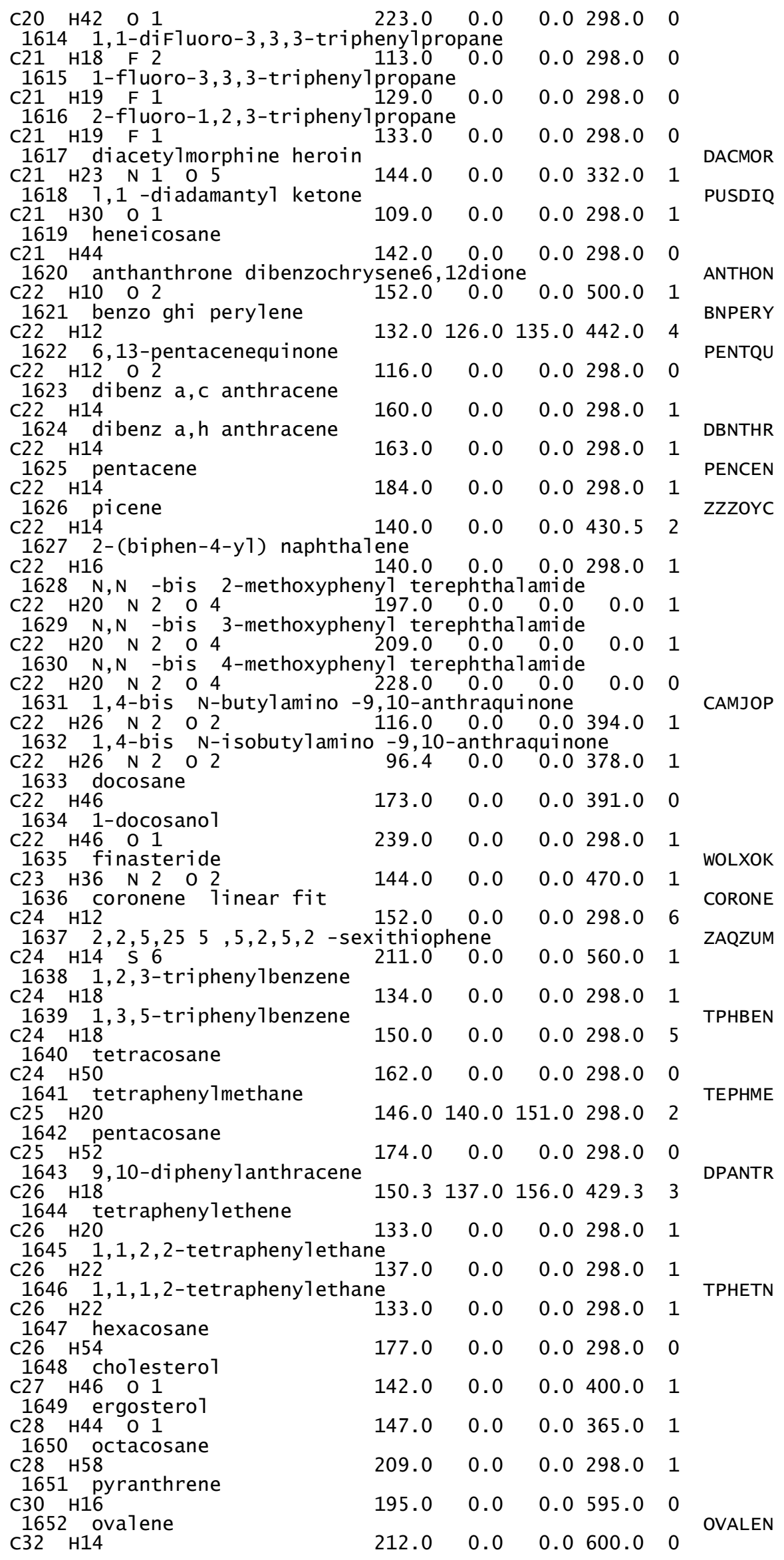




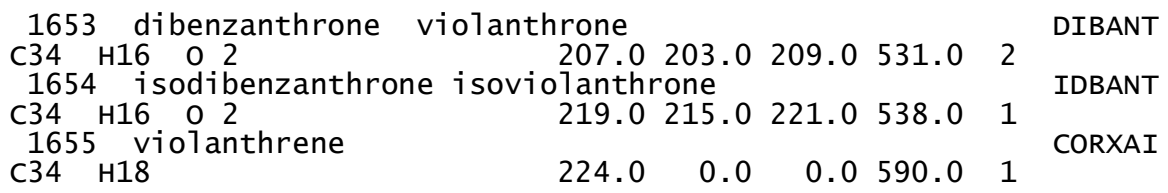

Table S2. Chemical composition of the 1655-data sample. Number of molecules in which each atomic species is present, and number of compounds in selected chemical classes. Compounds belonging to more than one class were assigned according to the most prominent functionality..

\begin{tabular}{|c|c|c|c|}
\hline atomic species & $\begin{array}{l}\text { number of } \\
\text { molecules }\end{array}$ & chemical class & $\begin{array}{l}\text { number } \\
\text { of entries }\end{array}$ \\
\hline $\mathrm{H}$ & 1611 & $\begin{array}{l}\text { amines, amides, } \\
\text {-aza compounds }\end{array}$ & 365 \\
\hline $\mathrm{C}$ & 1655 & hydrocarbons & 192 \\
\hline $\mathrm{N}$ & 783 & $\begin{array}{l}\text { DNA bases } \\
\text { and uracils }\end{array}$ & 74 \\
\hline $\mathrm{O}$ & 1113 & acids & 104 \\
\hline $\mathrm{S}$ & 120 & alcohols & 68 \\
\hline $\mathrm{P}$ & 7 & nitro & 129 \\
\hline $\mathrm{F}$ & 58 & & \\
\hline $\mathrm{Cl}$ & 174 & & \\
\hline $\mathrm{Br}$ & 46 & & \\
\hline I & 21 & & \\
\hline
\end{tabular}

Table S3. Attempted fitting of SE data $\left(\mathrm{kJ} \mathrm{mol}^{-1}\right)$ from different sources against temperature.

\begin{tabular}{|l|l|l|l|l|l|l|}
\hline compound & npts $^{\mathrm{a}}$ & nout $^{\mathrm{b}}$ & $\mathrm{T} \mathrm{range}^{\mathrm{c}}$ & $\Delta \mathrm{Cp}^{\mathrm{d}}$ & $\mathrm{SE}_{0}^{\mathrm{e}}$ & $\mathrm{SE}(298)$ or range $^{\mathrm{f}}$ \\
\hline & & & & & & \\
\hline coronene & 6 & 1 & $383-473$ & -0.071 & 173 & 152 \\
\hline p-terphenyl & 4 & 0 & $298-397$ & -0.050 & 138 & 123 \\
\hline hexachlorobenzene & 10 & 3 & $278-480$ & -0.082 & 124 & 100 \\
\hline 4-nitroaniline & 8 & 2 & $298-362$ & -0.041 & 113 & 101 \\
\hline acridine & 9 & 0 & $298-430$ & -0.051 & 108 & 93 \\
\hline tetracyanoquinodimethane & 5 & 0 & $413-500$ & -0.223 & 214 & 147 \\
\hline benzoic acid & 36 & 3 & $298-400$ & -0.035 & 101 & 91 \\
& 39 & 12 & $298-400$ & - & - & $88-91$ \\
\hline benzene & 12 & 0 & $193-298$ & -0.014 & 49 & 44 \\
& 12 & 5 & $261-298$ & - & - & $44-46$ \\
\hline anthracene & 31 & 3 & $298-450$ & -0.011 & 102 & 99 \\
& 31 & 3 & & - & - & $92-103$ \\
& 31 & 14 & & - & - & $95-100$ \\
\hline acenaphthene & 8 & 0 & $298-366$ & -0.030 & 94 & 85 \\
\hline & & & & & & \\
\hline
\end{tabular}

${ }^{a}$ Number of available data. ${ }^{b}$ Number of outliers excluded. ${ }^{c}$ Temperature range of data. ${ }^{\mathrm{d}}$ Slope of the least-squares line. ${ }^{\mathrm{e}}$ Intercept of the fitting line. ${ }^{\mathrm{f}} \mathrm{SE}(298)$ from fit, or range without fit. 
Table S4. First 1ine: sequence number as in Table S1, CSD refcode, compound name Second 1ine: sublimation enthalpy, Pixel lattice energy, Dabsolute, D\%;

AA-CLP lattice energy, Dabsolute, D\%. kJ/mol units.

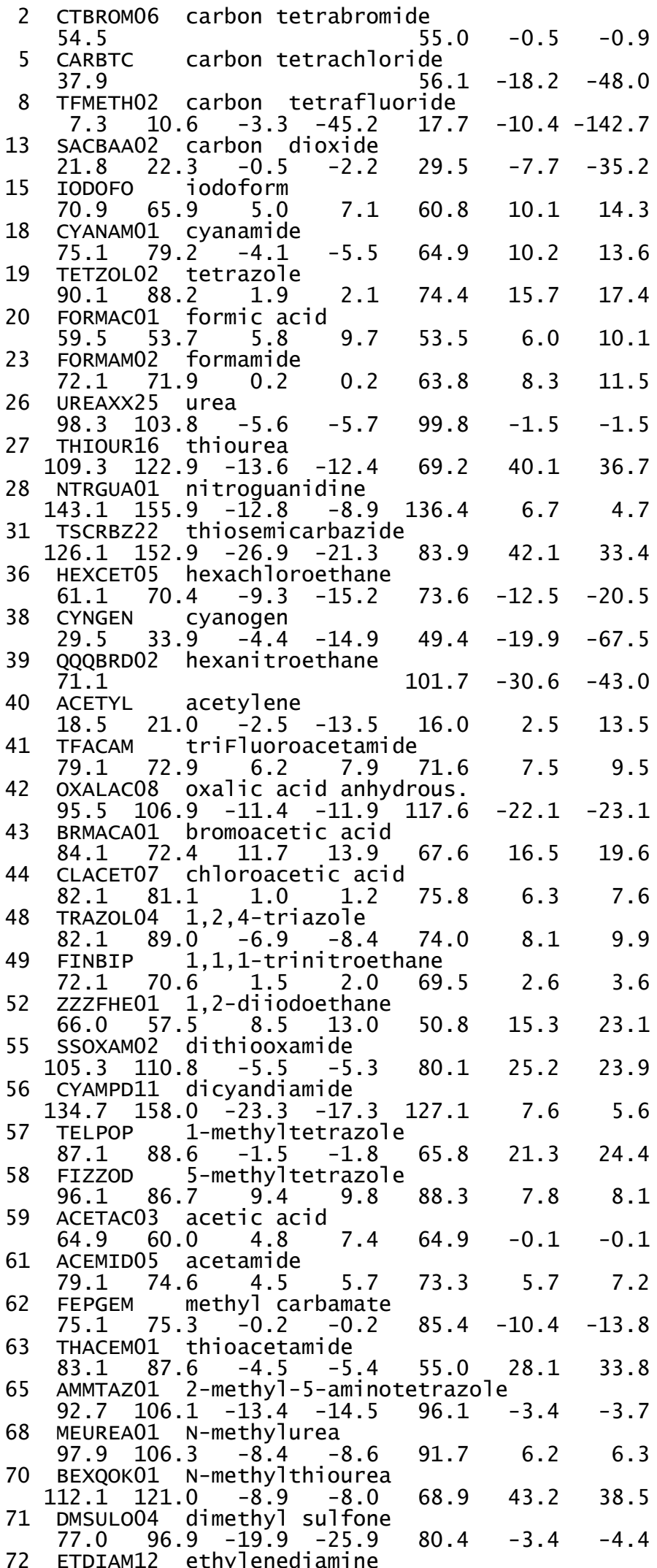




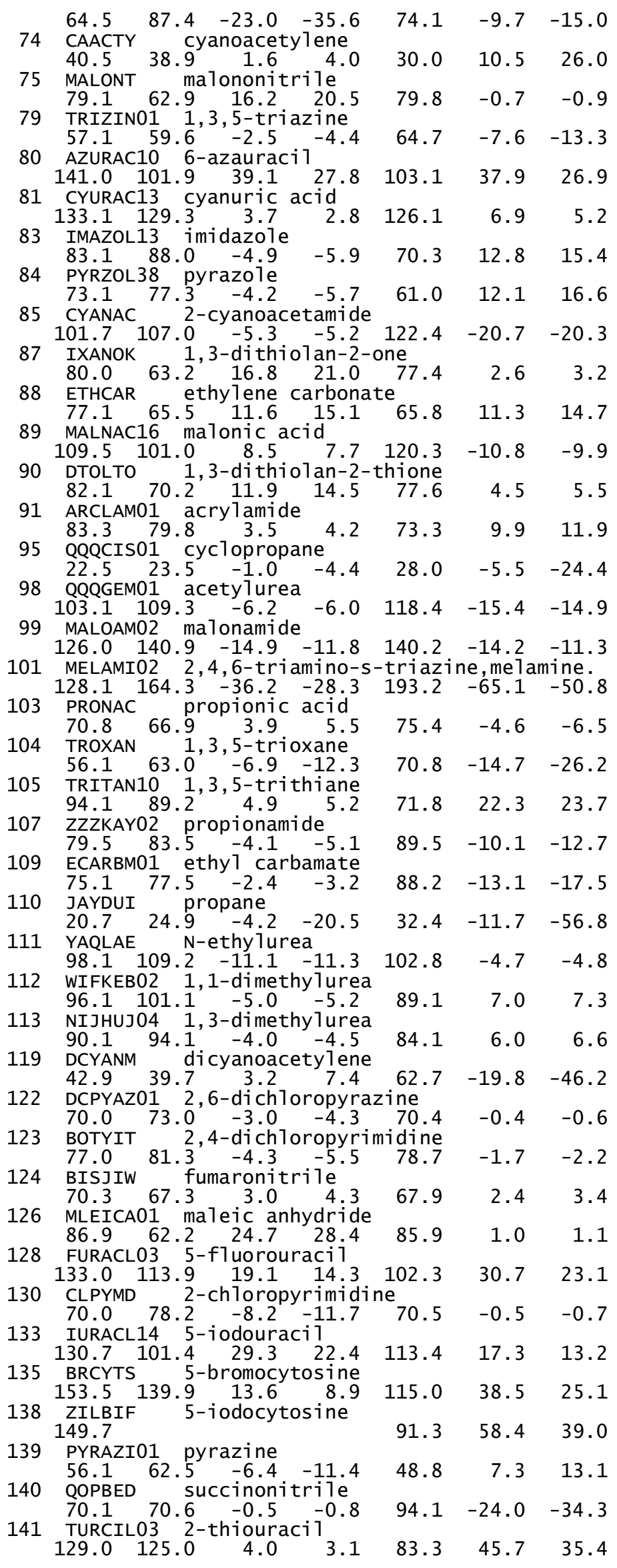




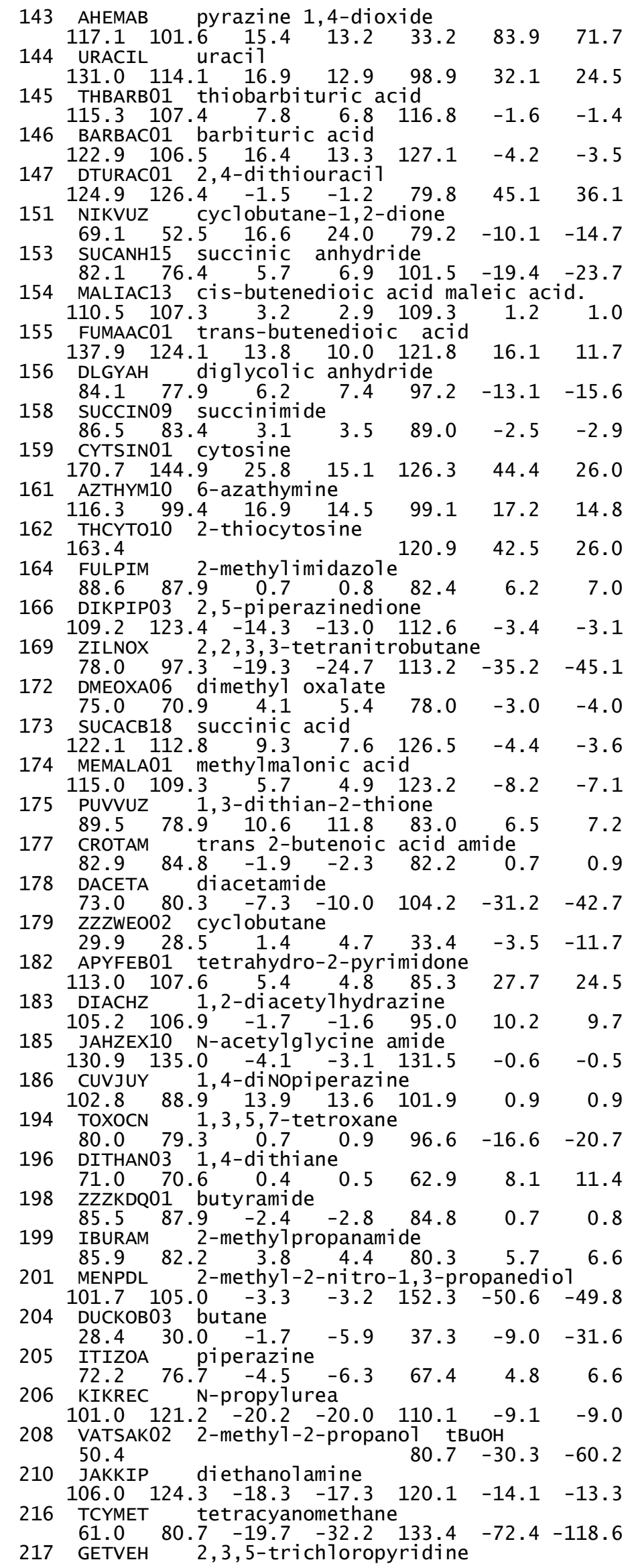




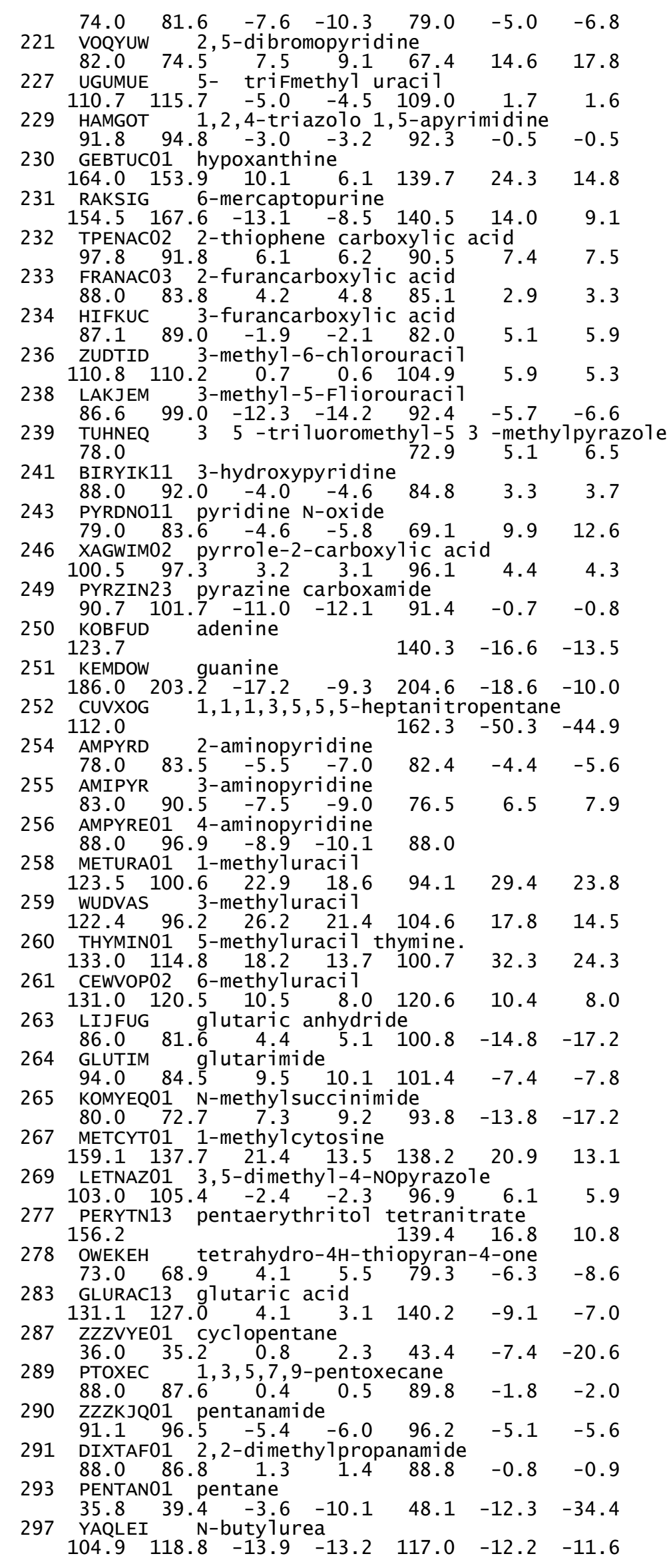




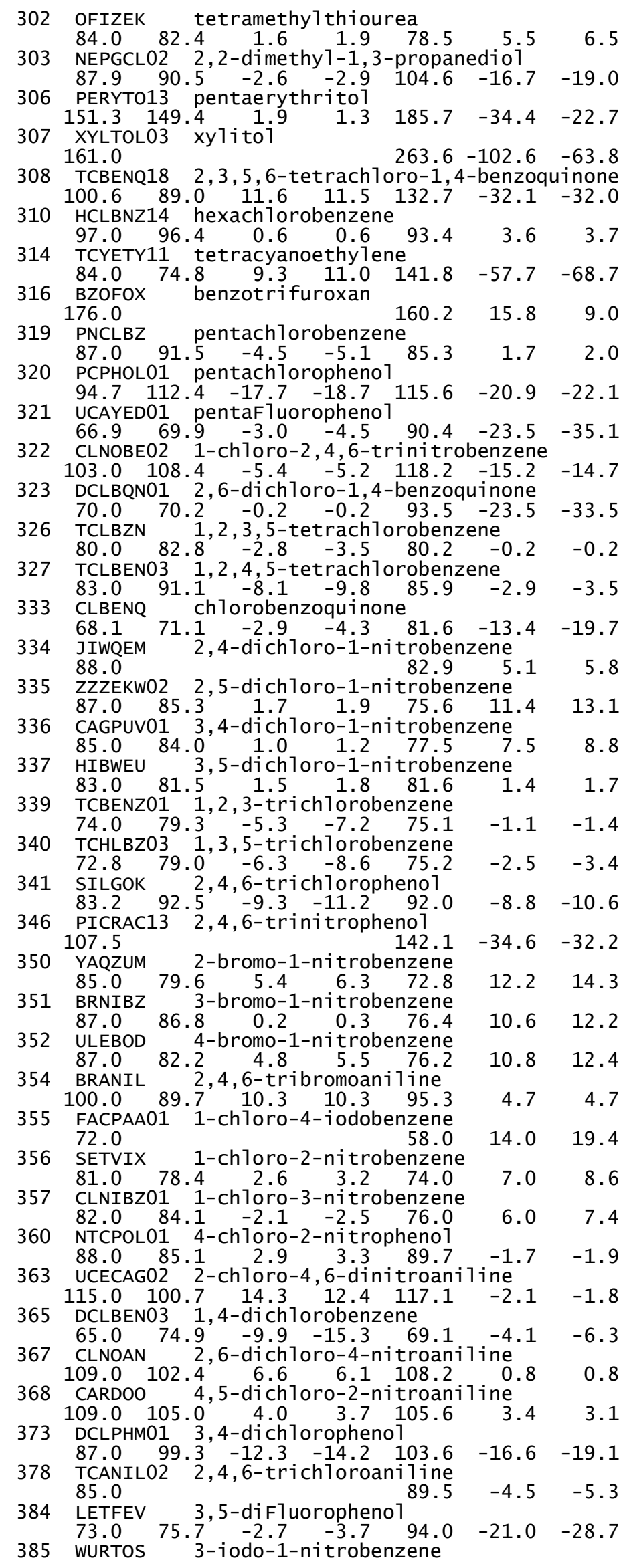




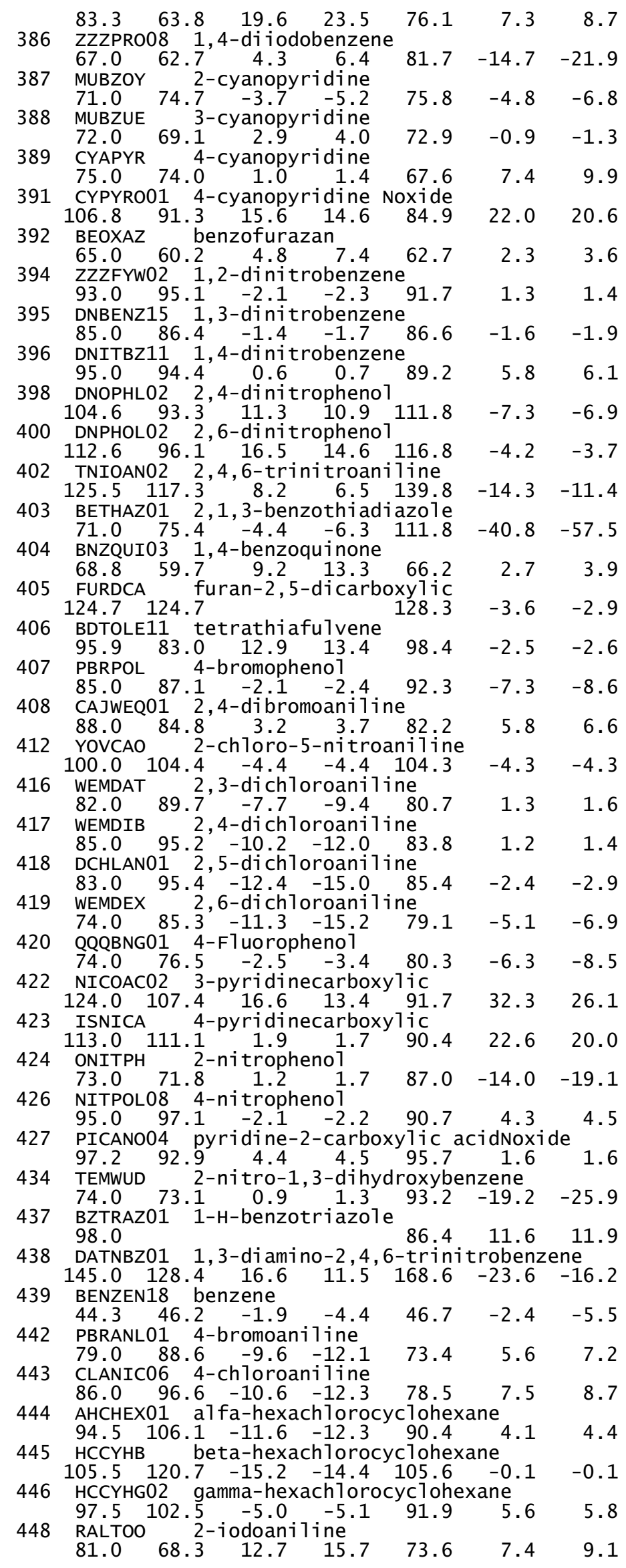




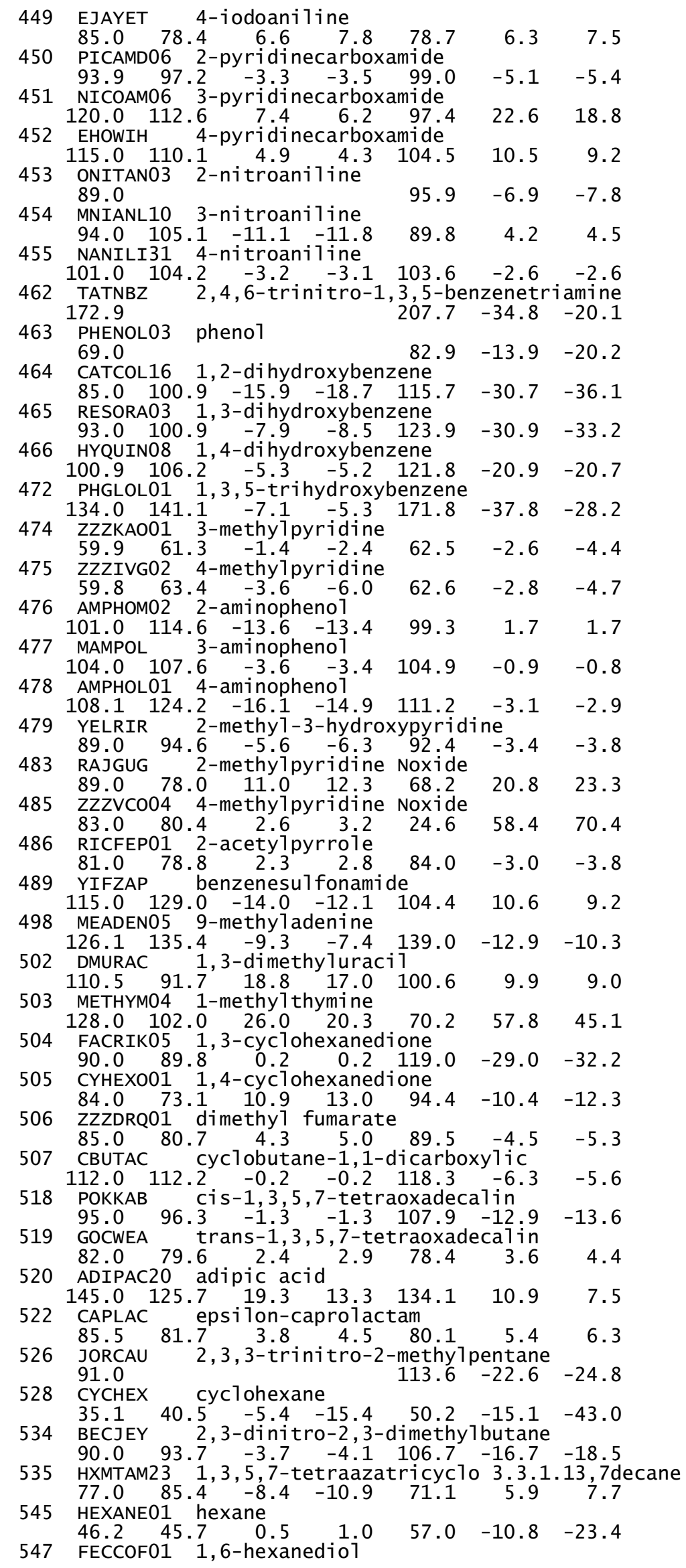




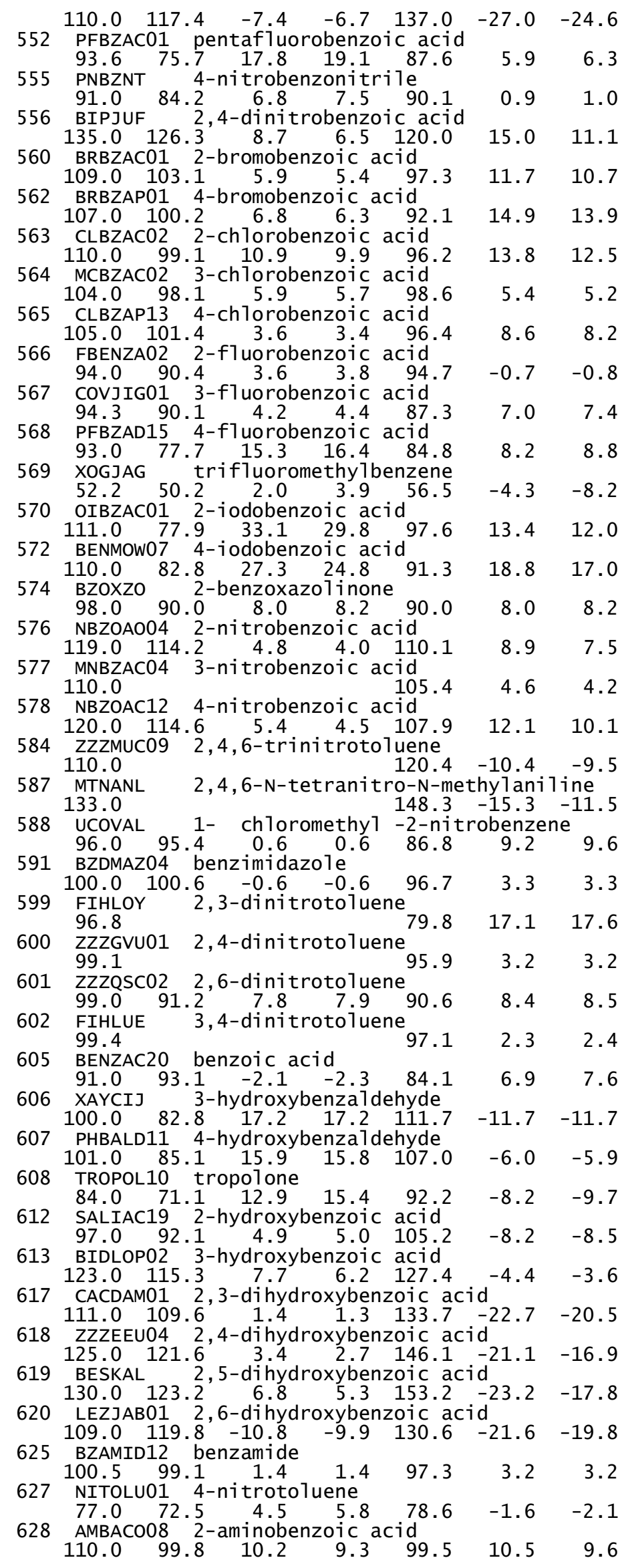




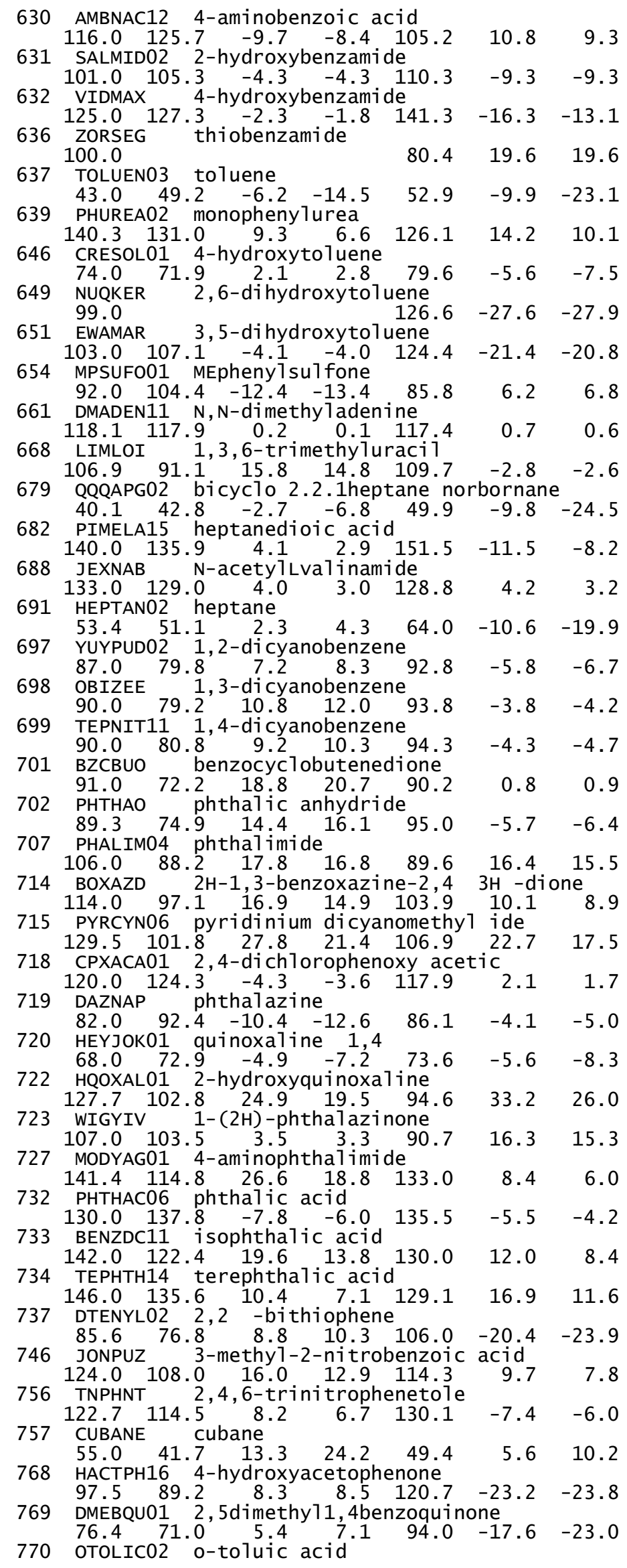




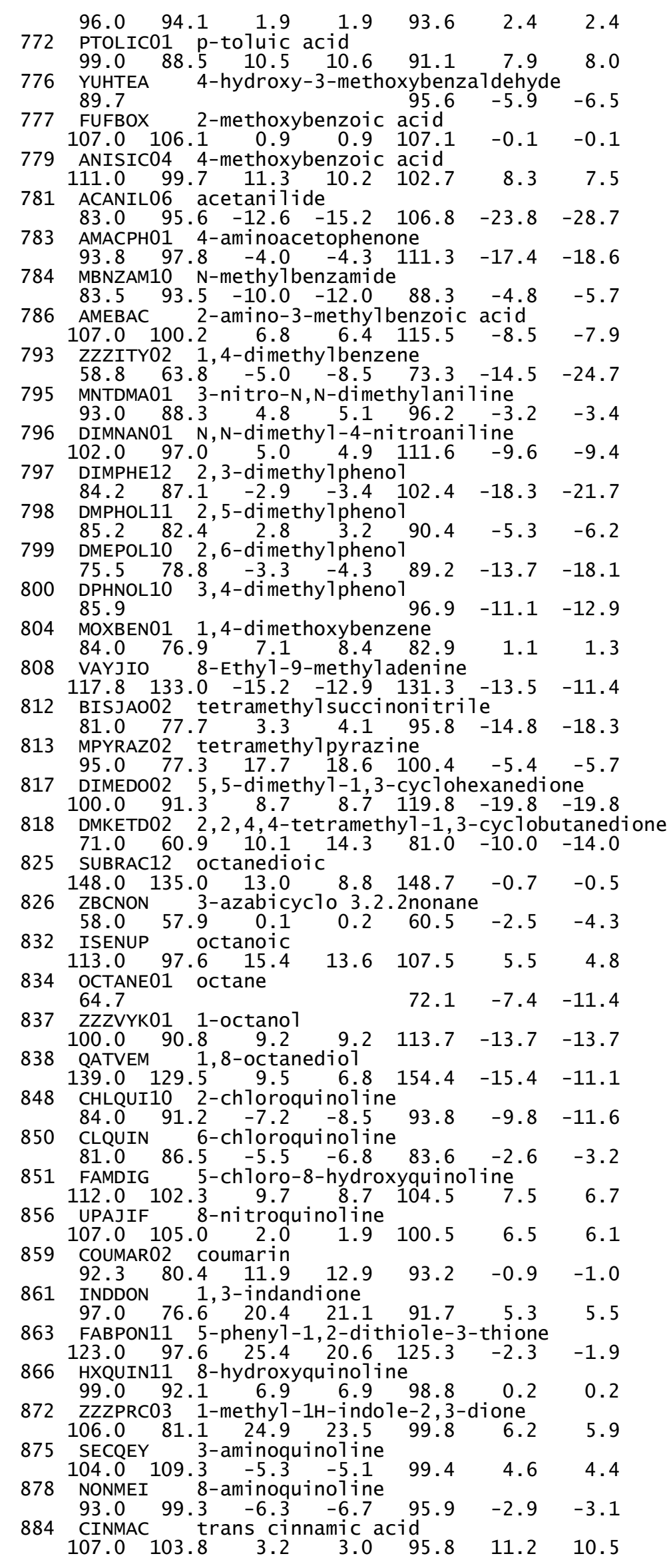




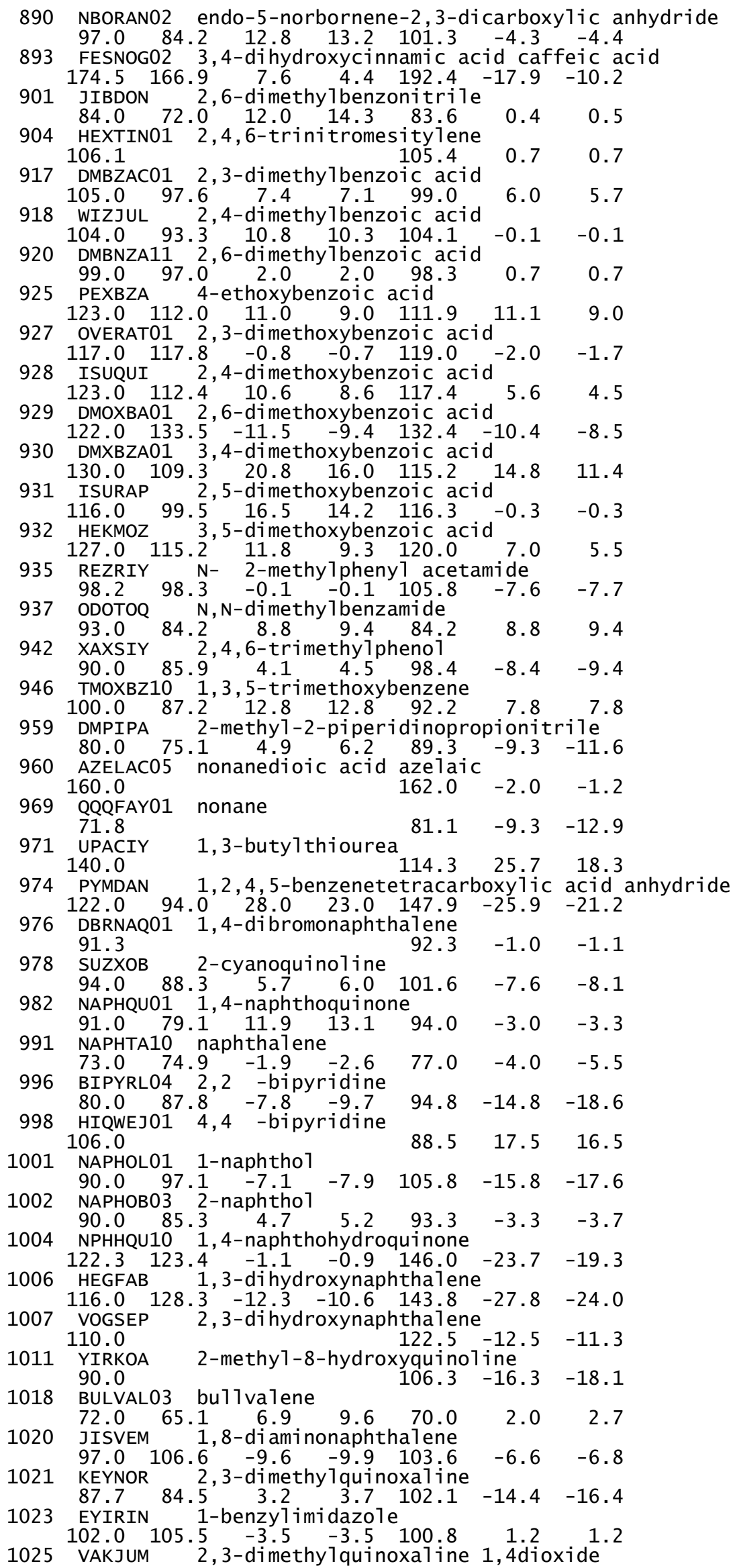




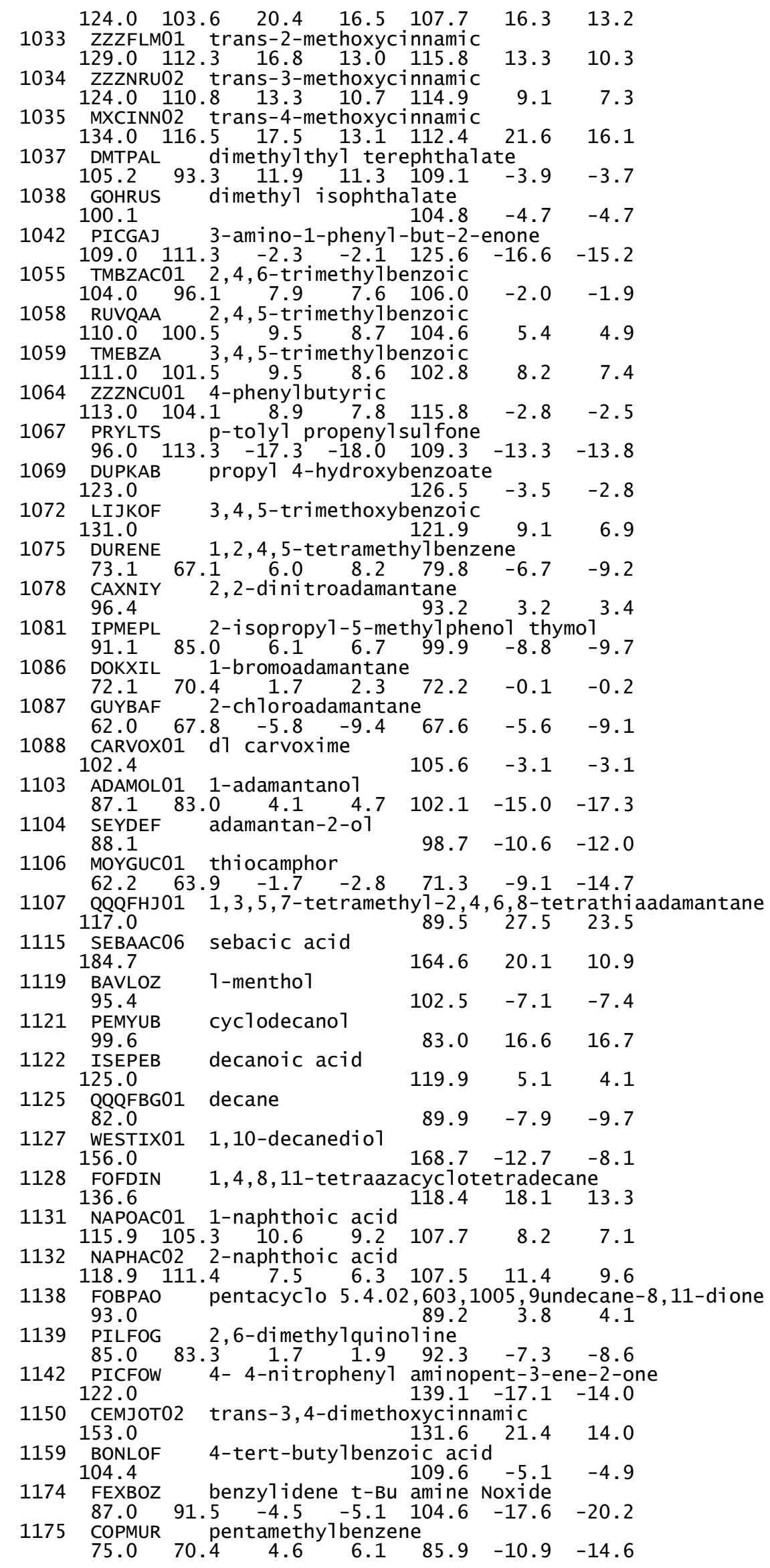




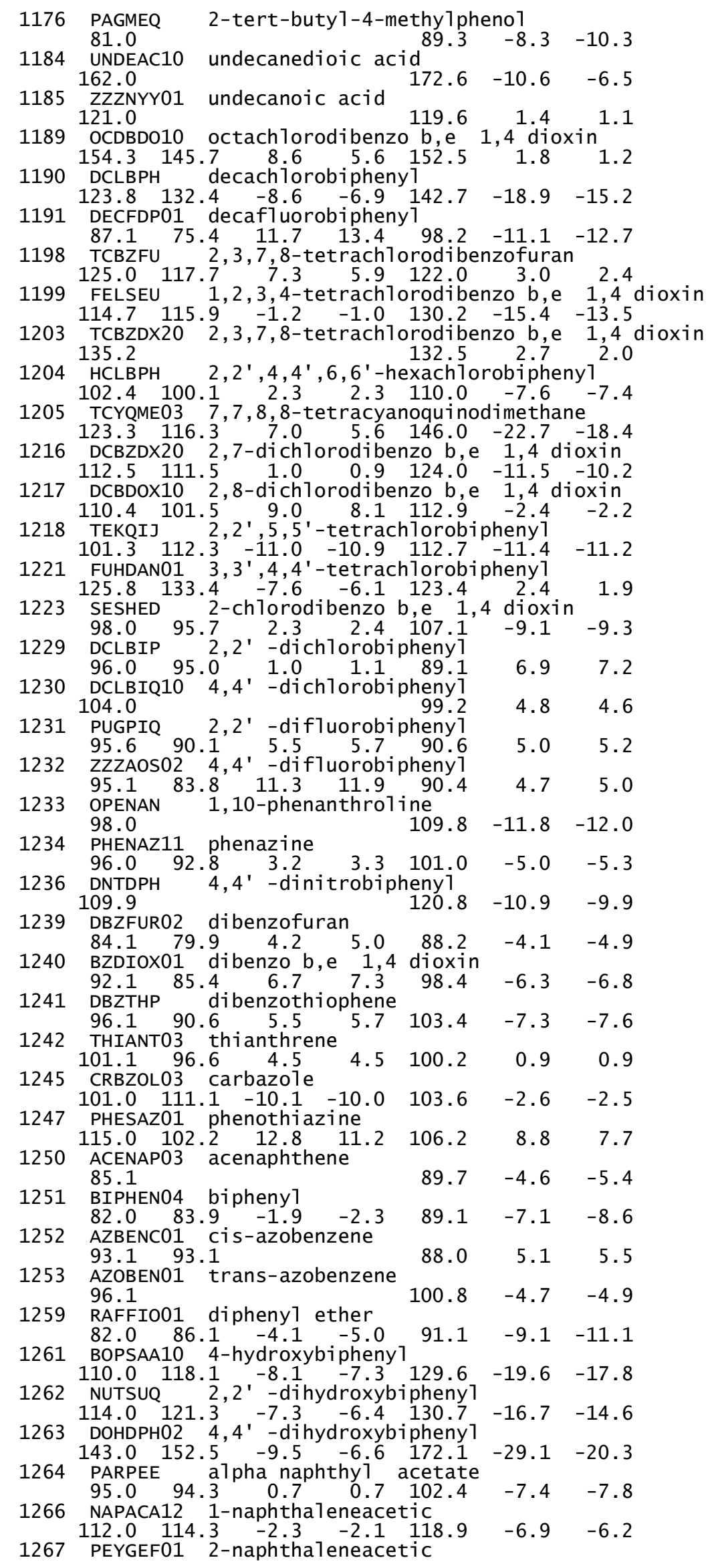




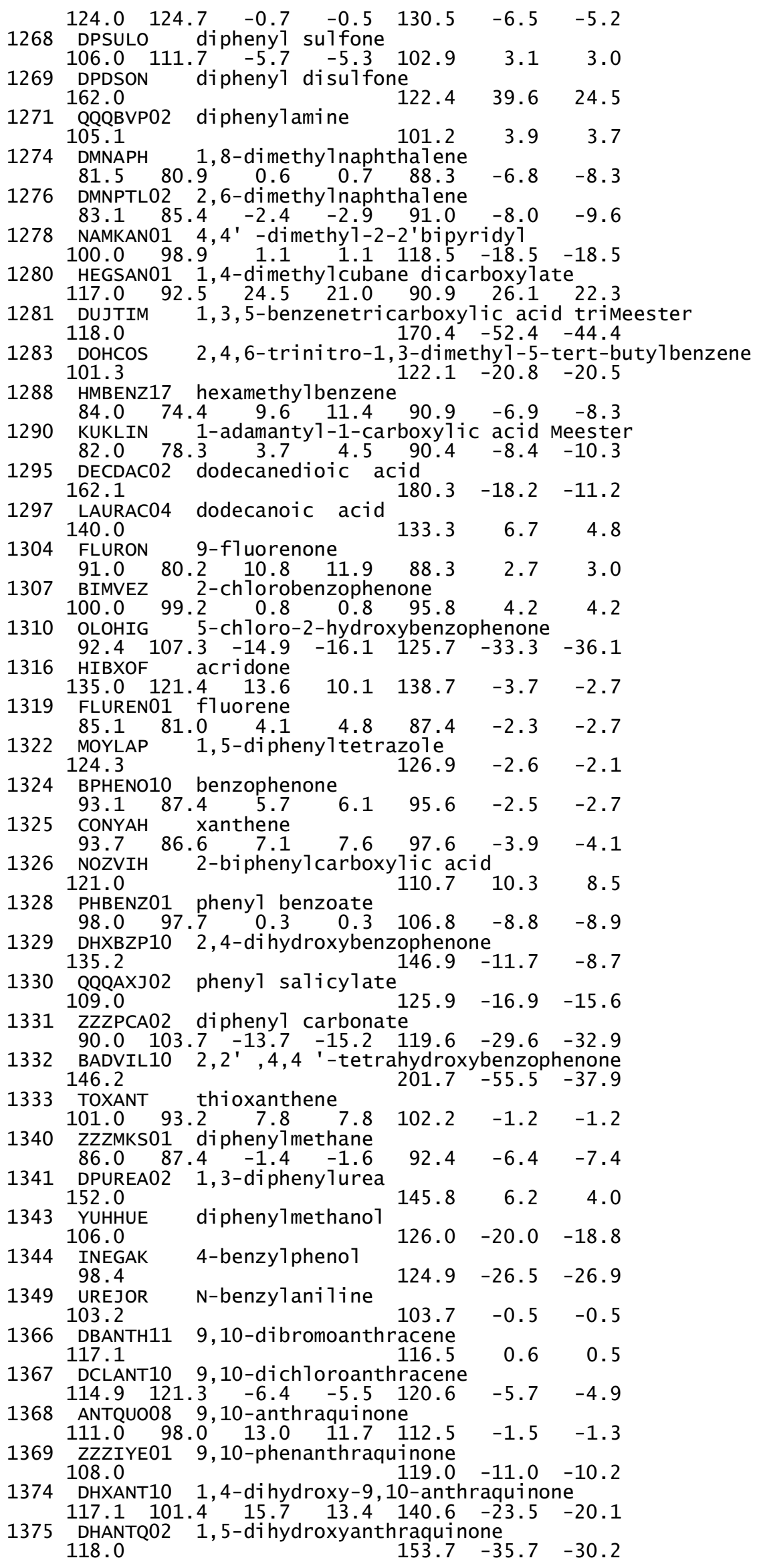




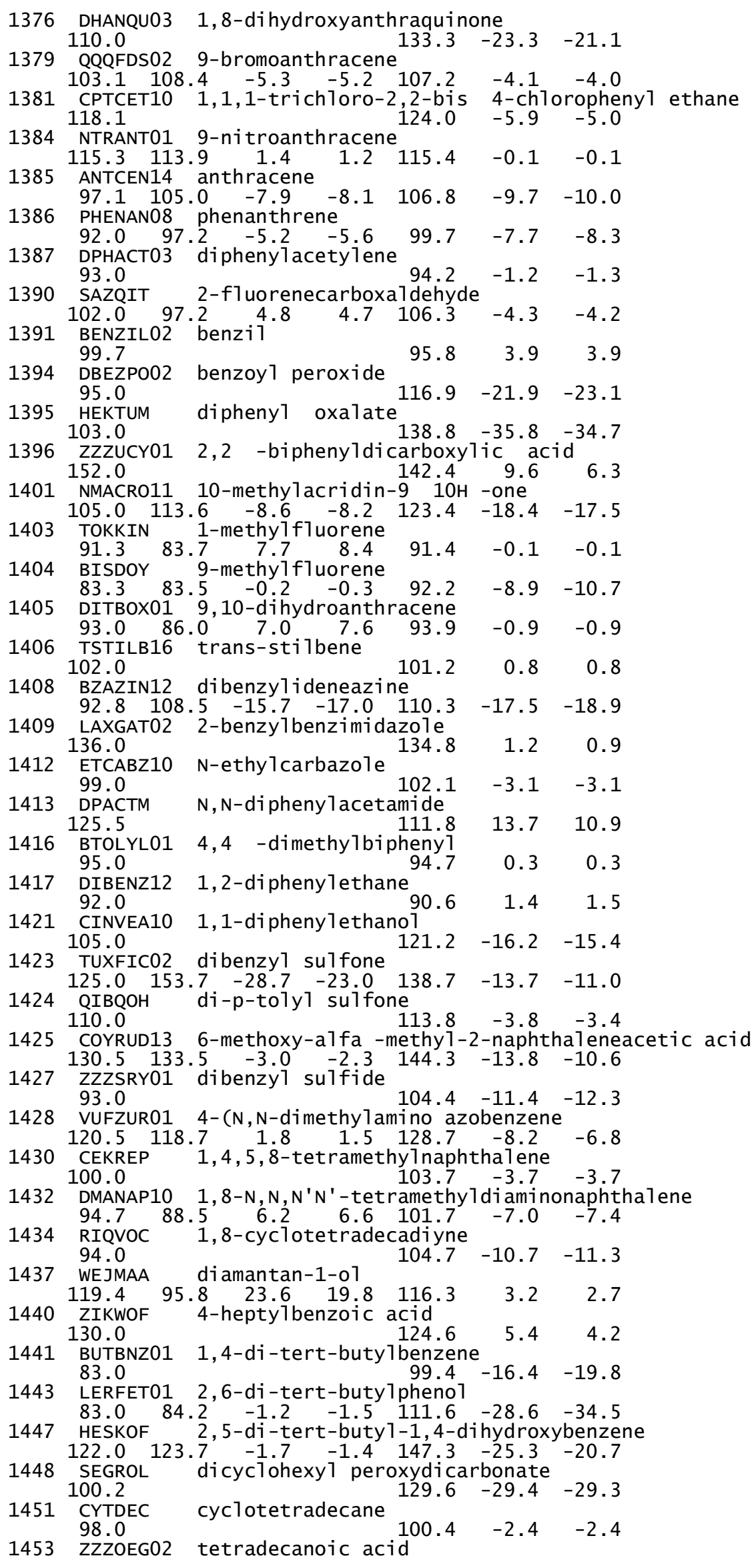




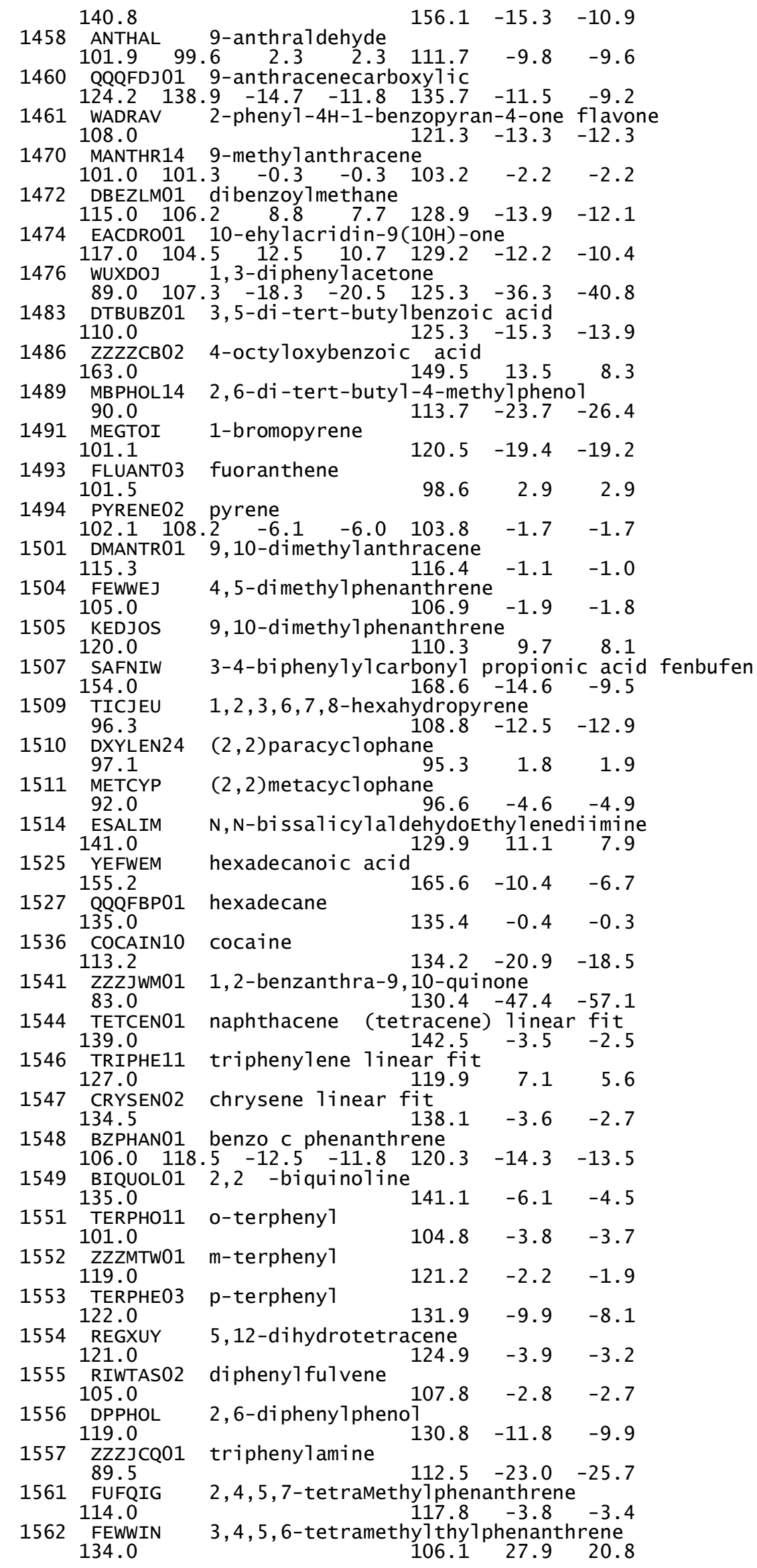




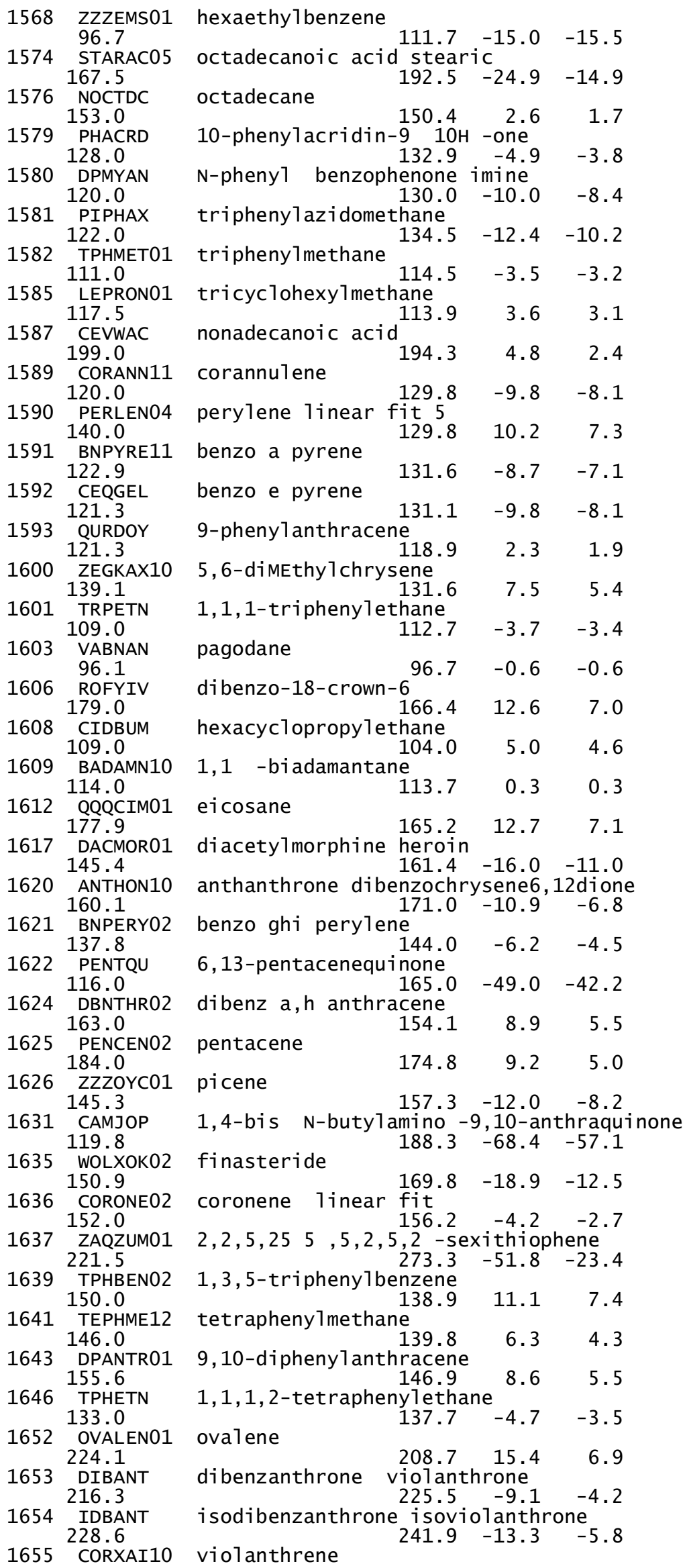


Figure S1. Plot of AA-CLP calculated lattice energies vs. experimental sublimation enthalpies.

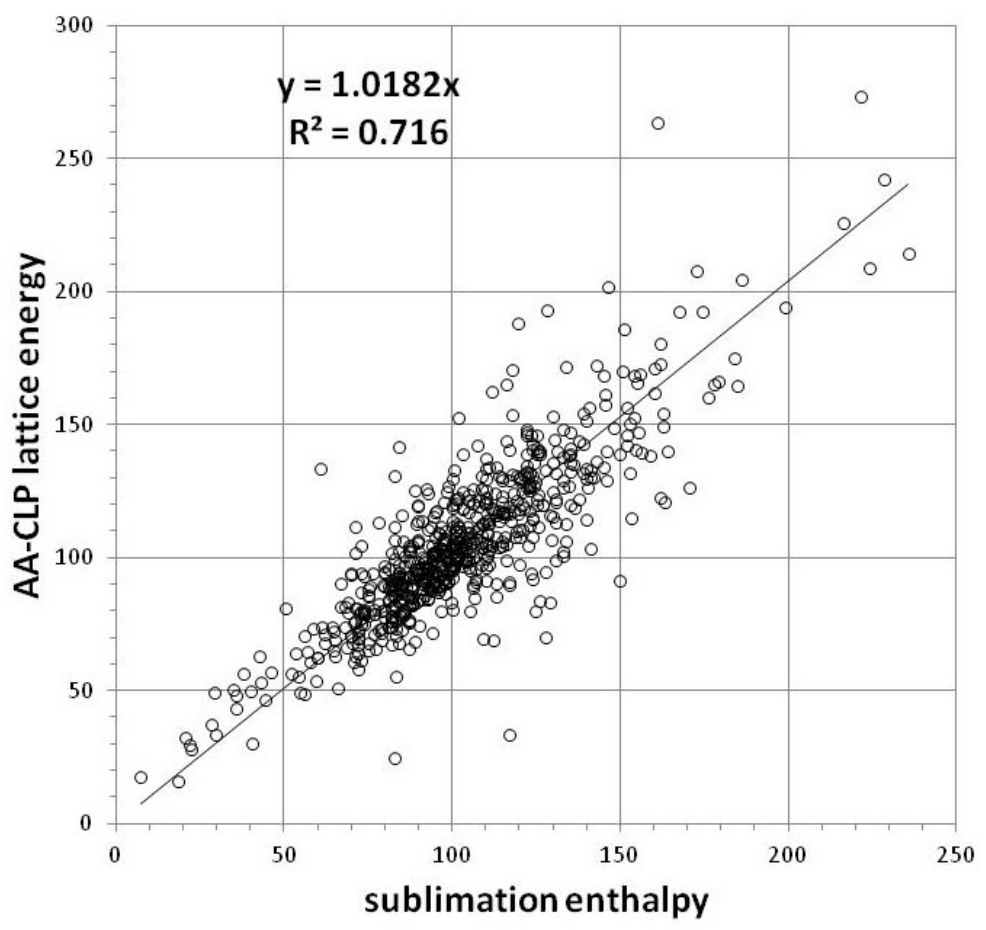

